\title{
Effects of stress on the GABAergic system in the hippocampal formation and medial prefrontal cortex of the adult male rat
}

\author{
PhD Thesis \\ in partial fulfilment of the requirements \\ for the degree "Doctor of Philosophy (PhD)" \\ in the Neuroscience Program \\ at the Georg August University Göttingen, \\ Faculty of Biology
}

submitted by

Wen $\mathrm{Hu}$

born in

Anqing, China 
First member of the Ph.D. Committee (Advisor):

Prof. Dr. Gabriele Flügge

Clinical Neurobiology Laboratory, German Primate Center (DPZ)

Second member of the Ph.D. Committee:

Prof. Dr. Ralf Heinrich

Institute for Zoology and Anthropology, University of Göttingen

Third member of the Ph.D. Committee:

Prof. Dr. Hubertus Jarry

Department of Clinical and Experimental Endocrinology, University of Göttingen 


\section{Declaration}

I hereby declare that this submission is my own work and that, to the best of my knowledge and belief, it contains no materials written by another person nor material which to a substantial extent has been accepted for the award of any other degree of the university or other institute of higher education, except where due acknowledgment has been made in the text.

Signature

Name

Date and place 


\section{Table of Content}

Abstract___ 4

Abbreviations __ 6

1. Introduction _ 8

1.1 The GABAergic interneurons ___ 8

1.2 The network oscillations___ 8

1.3 GABAergic interneurons and network oscillations ___ 9

1.4 Chronic stress and major depressive disorder ___ 11

1.5 Modulation of neuronal physiology by corticosteroids _ 12

1.6 Implications of chronic stress for neuronal morphology and physiology __ 13

1.6.1 Hippocampus _ 13

1.6.2 Medial Prefrontal Cortex _ 15

1.7 Chronic stress regulates the GABAergic system in limbic brain areas ___ 16

1.8 The chronic restraint stress paradigm __ 17

1.9 Aims of the thesis__ 18

2. Materials and Methods 20

2.1 Experimental animals___ 20

2.2 Restraint stress __ 20

2.3 Electrophysiology

2.3.1 Brain slice preparation ___ 21

2.3.2 Whole-cell patch-clamping___ 21

2.3.3 Spontaneous and miniature IPSC recordings __ 22

2.3.4 Depolarization induced suppression of inhibition - DSI___ 23

2.3.5 Data analysis___ 23 
2.5 Immunocytochemistry 24

2.6 Quantification of CCK and PV-immunoreactive neurons in the hippocampus 25

2.7 Stereology - Quantification of PV-immunoreactive neurons in the mPFC 25

2.8 Statistical analysis 27 28

3.1.1 Effects of the GR agonist dexamethasone on GABAergic transmission 28

3.1.1.1 Facilitation of hippocampal GABAergic transmission by the glucocorticoid dexamethasone 28

3.1.1.2 Dexamethasone effect mediated through a rapid non-classical GR mechanism 30

3.1.1.3 Facilitation of GABAergic transmission by dexamethasone through retrograde nitric oxide signaling

3.1.1.4 Facilitation of GABAergic transmission by dexamethasone partially through nitric oxide-induced release of CCK 34

3.1.2 Effects of acute stress on hippocampal GABAergic transmission 35

3.1.3 Effects of chronic stress on hippocampal GABAergic transmission 38

3.1.3.1 Physiological parameters after chronic restraint stress 38

3.1.3.2 $\mathrm{Ca}^{2+}$-dependent elevation of GABAergic transmission following chronic stress 39

3.1.4 Effects of chronic stress on the PV interneuron network in the hippocampus 42

3.1.4.1 Effects of chronic stress on numbers of PV- and CCK-immunoreactive neurons 42

3.1.4.2 Chronic stress effects on CCK-induced rhythmic burst activity originating from the PV interneurons 42

3.1.5 Effects of chronic stress on endocannabinoid mediated modulation of CCK interneuron activity in the hippocampus

3.2 Part II: Stress effects in the mPFC 51

3.2.1 A lateralized chronic stress effect on the number of PV-immunoreactive cells in the mPFC 51 
3.2.2 Lateralized effects of acute stress on the GABAergic transmission in the mPFC

3.2.3 Lateralized acute stress effects on sensitivity to DSI

3.2.4 Effects of varying dopamine concentrations on GABAergic transmission and DSI

sensitivity in the mPFC

4. Discussion 60

4.1 Part I. The effects of GR stimulation as well as stress in the hippocampus 60

4.1.1 Facilitation of GABAergic transmission by nongenomic glucocorticoid receptors 60

4.1.2 Stress effects on GABAergic transmission in the hippocampus 62

4.1.3 A functional deficit of the PV+ neuronal network induced by chronic stress 64

4.1.4 Summary: The nongenomic DEX action and the effects of chronic stress on the PV interneuron network 65

4.1.5 Effects of chronic stress on endocannabinoid mediated suppression of GABA release from CCK interneurons 67

4.2 Part II: Stress effects in the mPFC 69

4.2.1 A chronic stress-induced reduction in the number of PV-immunoreactive cells in the right MPFC

4.2.2 Lateralized effects of acute stress on the interneuron network within the mPFC 69

4.2.3 Dose-dependent actions of dopamine 70

4.2.4 Lateralized dopamine release and acute stress response 74

4.2.5 Presumptive functional implication 74

4.3 Summary and conclusions 75

References 77

List of tables and figures 94

Acknowledgements 96

Curriculum vitae 97 


\section{Abstract}

Stress facilitates the development of psychiatric disorders in vulnerable individuals. Previous studies have well documented the impact of stress on glutamatergic excitatory transmission in limbic brain regions, but little is known about stress effects on the GABAergic network. The aims of the present thesis were 1) to investigate effects of acute as well as chronic restraint stress on activities of interneurons in the hippocampal formation and the medial prefrontal cortex (mPFC) of adult male rats; and 2) to identify the neurophysiological mechanisms which may contribute to potential pathogenetic processes of chronic stress.

In the first part of this thesis, effects of stress and the synthetic glucocorticoid dexamethasone (DEX) on hippocampal GABAergic transmission and network function were studied, focusing on two perisomatic interneurons, the parvalbumin (PV) and the cholecystokinin (CCK)-expressing interneurons. Whole-cell voltage clamping of CA1 pyramidal neurons of acute hippocampal slices revealed a rapid increase of spontaneous inhibitory postsynaptic currents (sIPSCs) following bath application of the potent glucocorticoid receptor agonist DEX. This effect of DEX was mediated by a nongenomic glucocorticoid receptor which evoked nitric oxide (NO) release from pyramidal neurons. Retrograde NO signaling caused the augmentation of GABA release from the interneurons and additionally increased CCK release from CCK interneurons which in turn further enhanced the activity of the PV interneurons. Chronic restraint stress also resulted in a $\mathrm{Ca}^{2+}$-dependent increase in sIPSCs in CA1 pyramidal neurons and an additional DEX application elicited no further effect. Concomitantly, chronic stress reduced the number of PV-immunoreactive cells and impaired rhythmic sIPSCs originating from the PV-positive interneurons. In contrast, these parameters of CCK interneurons remained unaffected. Because CCK specifically stimulates the PV interneurons it is proposed here that, in addition to the immediate effect, the sustained activation of nongenomic glucocorticoid receptors during chronic stress may eventually injure the PV-interneuron network and lead to its functional impairment observed in this study. Furthermore, chronic restraint stress resulted in a dysfunction in CB1 mediated modulation of GABAergic transmission in the hippocampus. In acute hippocampal slices, depolarization induced suppression of inhibition (DSI; a form of short term plasticity at the GABAergic 
synapses known to be CB1 mediated and suggested to be involved in hippocampal information encoding) was impaired by chronic restraint stress. Because hippocampal CB1 receptors locate almost exclusively on the CCK interneurons, these data demonstrate altered functioning of the CCK interneurons following chronic stress.

Taken together, chronic stress had differential impacts on the structural and functional integrity of the CCK and PV interneurons in the hippocampus and, probably as a consequence, resulted in an imbalance in perisomatic inhibition mediated by the PV and CCK interneurons. The stress-induced dysfunctional inhibitory network may in turn impair rhythmic oscillations and thus lead to cognitive deficits that are common in animal models as well as in patients with stress-related psychiatric disorders.

The second part of this thesis describes effects of restraint stress in the mPFC with a discrimination of the right and left hemisphere. Three weeks of chronic stress decreased the number of PV-immunoreactive interneurons in the right $\mathrm{mPFC}$ while the number of $\mathrm{PV}$ cells in the left mPFC was unaffected. Whole-cell voltage clamp recording in layer II/III pyramidal neurons in acute PFC slices showed that $30 \mathrm{~min}$ of acute stress increased sIPSC frequency specifically in the right mPFC but induced DSI sensitivity specifically in the left mPFC. Both effects of acute stress could be abolished in the presence of a dopamine D1 receptor antagonist. Furthermore, acute application of dopamine mimicked the observed effect in a dose-dependent manner. Whereas a low concentration of dopamine evoked enhanced sIPSC frequency, mimicking the stress effect in the right mPFC, a higher concentration induced DSI sensitivity, mimicking the stress effect in the left mPFC. This is in consistence with previous reports showing that a brief restraint stress resulted in a left>right increase in dopamine release in the mPFC. These findings provide preliminary physiological evidences suggesting how the lateralized stress-induced dopamine release may contribute to the lateralized mPFC functioning which is probably necessary for efficient coping with acute environmental stressors.

In summary, the present thesis describes in detail the effects of chronic and acute restraint stress on the GABAergic system in hippocampal formation and mPFC. The results increase the understanding of how changes in the activity of the interneuron network may contribute to the responses to stress of varying durations. 


\section{Abbreviations}

7-NI 7-nitroindazole

ACSF artificial cerebrospinal fluid

AMPA a-amino-3-hydroxy-5-methylisoxazole-4-propionic acid

$\mathrm{BCV} \quad$ biological variance

BSA bovine serum albumine

CA1 Cornu Ammonis 1

CA3 Cornu Ammonis 3

CB1 cannabinoid receptor type 1

CBL calbindin

CCK cholecystokinin

CE coefficient of error

CORT corticosterone

CV coefficient of variation

D1R dopamine receptor of the D1 family

D2R dopamine receptor of the D2 family

(D)-AP5 D-2-amino-5-phosphonopentanoate

DEX dexamethasone

DSI depolarization induced suppression of inhibition

EGTA ethylene-glycoltetraacetic acid

EPSP excitatory postsynaptic potential

GR glucocorticoid receptor

HEPES N-hydroxyethylpiperazine- $N$ '-ethanosulphonic acid

HPA axis hypothalamus-pituitary adrenal axis

GABA $\quad y$-aminobutiric acid

GAD glutamic acid decarboxylase

mIPSC miniature inhibitory postsynaptic current

mPFC medial prefrontal cortex

MR mineralocorticoid receptor 


$\begin{array}{ll}\text { NMDA } & \text { N-methyl-D-aspartate } \\ \text { NOS } & \text { nitric oxide synthase } \\ \text { NOsGC } & \text { nitric oxide sensitive guanylyl cyclase } \\ \text { PV } & \text { parvalbumine } \\ \text { SD } & \text { standard deviation } \\ \text { SIPSC } & \text { spontaneous inhibitory postsynaptic current } \\ \text { SNAP } & \text { S-nitroso-N-acetylpenicillamine } \\ \text { TTX } & \text { tetrodotoxin }\end{array}$




\section{Introduction}

\subsection{The GABAergic interneurons}

Y-Aminobutyric acid (GABA) is the major inhibitory neurotransmitter in the mammalian brain, first identified during the 1950s (Roberts, 1988). Two types of GABA receptors are known. The $G A B A_{A}$ receptors are ionotropic and primarily conduct $\mathrm{Cl}^{-}$permeability. The $G A B A_{B}$ receptors are G-protein-coupled metabotropic receptors, located in both pre- and postsynaptic sites where they regulate transmitter release by reducing $\mathrm{Ca}^{2+}$ influx and mediate slow hyperpolarization by activating $\mathrm{K}^{+}$current, respectively (Ge et al., 2007).

There is a large diversity of GABAergic interneuron subtypes in the cerebral cortex, defined and recognized on the basis of the discriminated morphological as well as electrophysiological properties and expression pattern of molecular cell markers (Markram et al., 2004; Somogyi and Klausberger, 2005). In the hippocampal CA1 area, 16 distinct types of GABAergic interneurons have been identified (Somogyi and Klausberger, 2005). Each domain of a pyramidal neuron is innervated by distinct subtypes of interneurons which sub-serve specific functional roles in regulating the activity and plasticity of the target cell (Markram et al., 2004). Basket cells and axo-axonal chandelier cells are those who target onto the perisomatic domain of a pyramidal cell including the cell body, the proximal dendrites up to a distance of $\sim 100 \mu \mathrm{m}$ from the soma and the initial segment of the axon (Megías et al., 2001; Papp et al., 2001). These neurons have the ability to precisely and potently control the timing of action potential generation. By contrast, interneurons terminating on the distal dendrite are more suited to modulation of input signals by way of shunting (Miles et al., 1996).

\subsection{The network oscillations}

The coordinated oscillatory activity in various brain areas is a hallmark of neuronal netrwork function and is believed to provide temporal structure that is necessary for cooperating neuronal assemblies to perform specific functions such as representation, information processing, memory storage and retrieval etc. (Buzsáki and Draguhn, 2004; Somogyi and 
Klausberger, 2005).

Network oscillations of different frequencies are thought to represent distinct brain states. For instance, fast oscillations in the gamma band $(30-93 \mathrm{~Hz})$ have been proposed to be associated with higher brain functions such as those involved in working memory (Howard et al., 2003). In rat, hippocampal theta rhythmicity is observed when an animal is actively engaged in locomotor behavior such as walking, orienting or spatial exploration, and also present during REM sleep (Vanderwolf, 1969; Buzsáki, 2002). A deficit in the synchronization of cortical neuronal assemblies may lead to a disruption of network oscillations which consequently results in the clinical symptoms such as in patients with stress-related psychiatric disorders or behavioral deficits in animal models of those diseases. Indeed, it has been suggested that the functional deficit of the parvalbumin-expressing interneurons may contribute to disturbed gamma oscillation and consequently to the reported working memory dysfunction in schizophrenia patients (Lewis, et al 2005; Lodge, et al 2009).

\subsection{GABAergic interneurons and network oscillations}

It is generally agreed that diverse subtypes of GABAergic interneurons provide networks of inhibition which sculpt the firing pattern of pyramidal cells and orchestrate the precisely synchronized network oscillations (Buzsáki and Draguhn, 2004; McBain and Fisahn, 2001; Somogyi and Klausberger, 2005). Moreover, the GABAergic basket and chandelier cells that innervate the perisomatic domain of principal neurons play especially pivotal roles in generating synchronized network oscillations (Somogyi and Klausberger, 2005; Klausberger, et al 2005). Driven by external and internal stimuli these neurons evoke rhythmic perisomatic inhibitions in principal neurons to accurately control the timing of firing and to synchronize the activity of a large number of pyramidal cells (Somogyi and Klausberger, 2005; Klausberger, et al 2005; Sohal, et al 2009).

In the rat hippocampal formation, a brain area that is often investigated as a model structure for studying the cortical network, the two major types of perisomatic interneurons have been substantially described: the parvalbumin (PV) and the cholecystokinin (CCK)-positive interneuron. These two interneuron subtypes probably play different roles in the inhibitory 
network because of their distinct membrane properties, expression patterns of receptors and their presynaptic modulations (Klausberger, et al 2005; Glickfeld and Scanziani, 2006; Freund and Katona, 2007). Accordingly, compared to the CCK interneurons, the PV interneurons have shorter membrane time constants and lower input resistance (Glickfeld and Scanziani, 2006). Moreover, the PV interneurons receive higher magnitude of both excitatory and inhibitory inputs than CCK interneurons (Glickfeld and Scanziani, 2006). Therefore, whereas the PV interneuron may be able to faithfully follow high-frequency repetitive stimulation without accommodation, the CCK interneurons are more suited to act as integrators, summating consecutive inputs from two or more distinct afferents over much longer windows. Another important difference between these two types of interneurons is their distinct presynaptic modulation. CCK interneurons are distinguished from PV interneurons in that they represent the majority of subcortical afferent fibers and they express $5-\mathrm{HT}_{3}$ receptors, nicotinic receptors as well as presynaptic cannabinoid (CB1) receptors (Férézou et al., 2002; Freedman et al., 1993; Morales and Bloom, 1997; Porter et al., 1999). In the hippocampus, $\mathrm{CB} 1$ receptors are expressed almost exclusively on the axonal terminals of CCK interneurons (Hájos et al., 2000) and predispose these cells to retrograde, endocannabinoid-mediated modulation of GABA release which depends on the activity of targeting postsynaptic neuron, a phenomenon termed as depolarization induced suppression of inhibition (DSI, Maejima et al., 2001; Yoshida et al., 2002; Freund et al., 2003; Reich et al., 2005).

Because of these disparities, the functional dichotomy of the PV and CCK interneuons has been proposed recently: The PV+ interneuron syncytium is regarded as a non-plastic precision clockwork for network oscillations, while the CCK+ interneurons are considered to function as a plastic fine-tuning device that modulates synchronous activities as a function of subcortical inputs (Freund and Katona, 2007). Malfunctioning of the CCK+ inhibitory network has been suggested to contribute to emotional disorders including anxiety (Freund and Katona, 2007), although to the best of my knowledge this concept has not been tested experimentally. 


\subsection{Chronic stress and major depressive disorder}

Major depression is a major cause of disability in the developed countries. Worldwide it was the fourth leading cause of mortality and socioeconomic burden of disease in 2000 and is predicted to be the second leading cause after HIV in the year of 2030 (Mathers and Loncar, 2006). Despite intensive clinical and preclinical research, the etiology of depression as well as the neurophysiological processes that underlie its clinical symptoms is currently still not completely understood. It has been suggested that stressful life events confers a higher risk to the development of depressive episodes especially in vulnerable individuals who are genetically predisposed (Fava and Kendler, 2000; Caspi et al., 2003; Pittenger et al., 2008). Therefore, animal models of various stress paradigms have been employed and proven to be valuable tools to study the stress related neurobiological alterations that may underlie stress-related psychiatric disorders such as depression. For instance in humans, selective volume reduction in the hippocampus has been revealed in patients with major depression and post-traumatic stress disorder by imaging studies (Bremner, 2002; MacQueen et al., 2003; Sheline et al., 2003). Conversely, comparable hippocampal volume loss induced by chronic stress has also been documented (Czéh et al., 2001; Fuchs et al., 2004a; 2004b; Lee et al., 2009) and further investigations with animal models suggest that this structural plasticity is probably a manifestation of profound dendritic remodeling (Magariños and McEwen, 1995; Watanabe et al., 1992; Magariños et al., 1996) rather than loss of principle neurons (Vollmann-Honsdorf et al., 1997). In addition, using models of chronic stress, remarkable progress has been achieved toward the understanding of mechanisms underlying antidepressant actions (e.g. Alfonso et al., 2005; Abumaria et al., 2007; Schulte-Herbrüggen et al., 2009).

Any internal and/or external environmental challenges that disturb the homeostasis of an organism may be perceived as a stressor. The stress responses pe ser are not harmful or pathological. In fact a brief stress exposure can induce rapid adaptive physiological responses that enable an organism to react efficiently to the changing environment and therefore are beneficial for survival. For instance, recently it was reported that mice trained under more stressful conditions showed better learning and memory which was accompanied 
by enhanced synaptic expression of GluA2 AMPA receptors (Conboy and Sandi, 2010). However, when stress becomes repetitive and uncontrollable, a cascade of neuroplastic processes is initiated and eventually leads to marked morphological and functional alterations in the brain that resemble the pathophysiology observed in patients with stress related depressive disorders (reviewed in Joëls et al., 2007).

The stress response is mediated by the activation of two major systems: The first one is the hypothalamic-pituitary-adrenal (HPA) system which stimulates the adrenal cortex to release glucocorticoid, cortisol in primates and corticosterone in rodents, into the blood (Mason, 1968a). The other one is the sympathetic-adrenomedullary system which releases adrenaline from the chromaffin cells in adrenal medulla and noradrenaline from the sympathetic nerve endings, and triggers the flight-and-fight responses (Mason, 1968b). My work focuses on the former one.

\subsection{Modulation of neuronal physiology by corticosteroids}

Stress triggers enhanced release of circulating corticosteroids whose actions are mediated by two types of receptors in the brain: the low affinity glucocorticoid receptors (GRs) and the mineralocorticoid receptors (MRs) which have a ten-fold higher affinity for corticosterone (Reul and de Kloet, 1985; de Kloet et al., 1998). Both receptors are abundantly expressed in the limbic brain areas such as the hippocampal formation. The classical MRs and GRs belong to the family of nuclear receptors. During the inactive state, they reside in the cytoplasm and bound to chaperon molecules. Upon binding to corticosteroids, the receptors dissociate the chaperons, translocate to the nucleus and either directly bind to the response elements in the promoter region of responsive genes as hetero-/homodimers or interact with other transcription factors as monomers (Duma et al., 2006; Pascual-Le Tallec and Lombès M, 2005). Through influencing gene transcription, corticosteroids are able to exert potent modulation of neuronal excitability in a delayed but long-lasting fashion (Joëls et al., 2007; Joëls, 2008; Karst et al., 2005a). In addition, recently the existence of nongenomic actions of the MRs/GRs gained substantial credit and it was shown that corticosteroids can also rapidly affect neuronal function by engaging membrane bound, G protein associated receptors 
(Campolongo, et al 2009; $\mathrm{Di}$, et al 2005; 2009; Haller, et al 2008; Karst, et al 2005b;

Olijslagers et al., 2008). It was proposed by de Kloet et al. (2005) that the nongenomic and genomic signaling of the MRs and GRs collectively contribute to the adaptive stress responses: At the early stage, nongenomic corticosteroid signaling, together with other stress related compounds such as noradrenaline or corticotropin-releasing factor, rapidly enhances vigilance and alertness for effective coping. At the later stage through genomic corticosteroid actions, the cellular excitability normalizes which is necessary for dampening the initial stress responses and protecting the system from overshooting. Imbalanced regulation of this response system, due to chronic stress or genetic predisposition, may cause vulnerability to depressive like behaviors (de Kloet et al., 2005).

Because both MRs and GRs are expressed predominantly in principal cells, previous studies concerning corticosteroid effects on neuronal activity in the limbic brain areas especially in the hippocampal formation focused mainly on these neurons. However, whether and in which way GABAergic network functions are affected by the stress induced elevation of corticosteroids remains essentially unexplored. On the other hand, microdialysis studies report on increased extracellular GABA levels in the hippocampus of rats exposed to acute psychological stressor (de Groote and Linthorst, 2007). Stress or artificially elevated corticosterone levels have been shown to upregulate the expression of hippocampal GAD67 (glutamic acid decarboxylase, one of the enzymes responsible for the biosynthesis of GABA) (Bowers, et al 1998; Stone, et al 2001). Recently it was reported that in vitro, GR activation causes an increase in the magnitude of IPSCs in dorsal hippocampus (Maggio and Segal, 2009). These data indicate an interaction between the GABAergic transmission and corticosteroid signaling in response to stress and further investigation is required to understand the neuronal basis underlying this process and what is its functional significance.

\subsection{Implications of chronic stress for neuronal morphology and physiology}

\subsubsection{Hippocampus}

While short-term activation of the HPA axis is essential for optimal survival, persistent and 
repetitive stimulation due to excessive and prolonged stress is deleterious and may finally lead to exhaustion of the systems and possibly to pathophysiological processes. The most extensively studied brain structures with regard to effects of chronic stress is the hippocampal formation which plays an important in the negative feedback loop of the limbic-HPA axis and thereby in modulating of the release of the steroid hormones (de Kloet et al., 1998). Morphological alterations in the hippocampus due to chronic stress have been extensively documented. The reduction in the complexity of the apical dentritic tree of the CA3 pyramidal cells was observed after exposure to various chronic stress paradigms (Magariños and McEwen, 1995; Watanabe et al., 1992; Magariños et al., 1996) as well as after chronic administration of corticosterone (Woolley et al., 1990). A similar but less extensive dendritic remodeling of dentate granule cells and CA1 pyramidal cells was also reported (Sousa et al., 2000). In addition, chronic stress results in profound alterations in the ultrastructural of the mossy fiber synapses including a reduction in the volume of postsynaptic thorny excrescences (Stewart et al., 2005) and a rearrangement of presynaptic terminal structures (Magariños et al., 1997).

Moreover, chronic stress leads to marked changes in the hippocampal neurophysiology which is probably partially secondary to morphological changes since atrophy in the apical dendrites may result in a diminished number of spines receiving input signals, and it is known that the kinetics of remote synaptic events depend on dendritic morphology (Vetter et al., 2001). For instance, chronic social defeat stress in rats influences input signals of CA3 pyramidal neurons by shortening the onset latency of EPSPs (excitatory postsynaptic potentials) and increasing the input resistance, and these functional alterations significantly correlate with reduced surface area of the apical dendrites from the same neuron (Kole et al., 2004). Importantly, a background of previous chronic stress exposure may lead to a sensitized or enhanced response to both acute stress and in vitro activation of GRs/MRs. In the dentate gyrus of rats, chronic unpredictable stress produces no effect on the AMPA currents evoked by perforant path stimulation; however, in the chronically stressed animals but not in the controls, this responsiveness is significantly enhanced by corticosterone (Karst and Joëls, 2003). Conversely, the expression of both GRs and MRs in the hippocampus is regulated by chronic stress (Meyer et al., 2001; Paskitti et al., 2000). These changes in the 
neurophysiology of the hippocampus probably contribute to the various cognitive as well as emotional deficits and to enhanced vulnerability that are observed in animals experienced repeated stress.

\subsubsection{Medial Prefrontal Cortex}

The mPFC of the rat is implicated in a variety of cognitive functions such as task representation and memory consolidation (Miller, 2000; Euston et al., 2007). The mPFC also plays a unique role in the extinction of fear-related memory in that the mPFC reduces fear responses through its inhibitory projections to the amygdale (Akirav and Maroun, 2007; Milad and Quirk et al., 2002; Morgan et al., 1993). Moreover, during recent years, the key role of mPFC in the regulation of the stress responses has been largely acknowledged (for review see Czeh et al., 2008).

The mPFC receives extensive dopaminergic and noradrenergic innervations directly or indirectly from the ventral tegmental area and the locus coerulus, respectively. In addition, corticosteroid receptors were documented to be abundantly present in the mPFC of rodents and primates (Diorio et al., 1993). Under the condition of acute stress, the stress pathways in the HPA axis and brainstem are activated, evoking enhanced release of corticosteroids, noradrenaline and dopamine, and thereby the high order cognitive PFC functions such as working memory and attention regulation are affected (reviewed in Arnsten, 2009). On the other hand, similar to the hippocampus, mPFC contributes to the negative feedback control of the hypothalamus-pituitary-adrenal axis (Diorio et al., 1993; Herman et al., 2003). In addition, through reciprocal connections, mPFC may also regulate the stress responses of many subcortical areas (Amat et al., 2005; Pascucci et al., 2007).

Accumulating studies demonstrate that chronic stress leads to a selective atrophy of the apical dendritic arbors of the pyramidal neurons in layer II/III (Perez-Cruz C et al., 2007; 2009; Radley, 2004) as well as in layer $V$ of the mPFC (Liu and Aghajanjan, 2008). Using a combination of whole-cell recording and two-photon imaging in rat mPFC, it was recently demonstrated that the stress-induced morphological change correlates with diminished responses to apically targeted excitatory inputs (Liu and Aghajanjan, 2008). However, to my 
knowledge, whether and how at neuronal level the GABAergic system in the mPFC may be involved in stress related processes have not been investigated.

An important feature of PFC functioning is its hemispheric asymmetry (Carlson et al., 1991; 1993; Gratton et al., 2005; Perez-Cruz et al., 2007; 2009: Sullivan et al., 1999; 2004). With regard to stress induced dendritic remodeling, for instance, recently it was shown that the dendritic complexity of layer III pyramidal cells of rat mPFC exhibit an inherited hemispheric asymmetry with neurons in the right hemisphere having longer apical dendrites as compared to those in the left mPFC. 21 days of restraint stress affected mainly the right hemisphere; hence the inherent hemispheric asymmetry was abolished after stress (Perez-Cruz et al., 2007). Exact mechanisms underlying the lateralized stress responses are still unclear. Data from previous studies indicate that the right mPFC, which is more directly linked to areas involved in basic life-sustaining functions, is involved in integrating stress related sensory inputs with the appropriate autonomic as well as neuroendocrine output and is responsible for responses under long-term stressful situations (Sullivan and Gratton, 1999, 2002). By contrast, the left mPFC, which is more motor and less emotional, is more involved in the initial coping attempt (Sullivan, 2004). Importantly, it was suggested that the mesocortical dopaminergic pathway contributes significantly to this lateralized function of mPFC (Sullivan, 2004).

\subsection{Chronic stress regulates the GABAergic system in limbic brain areas}

Recently, a growing number of data indicates that the GABAergic system is also implicated in stress responses. Chronic stress and chronic glucocorticoid treatment modulate the expression of GAD67 mRNA (Bowers et al., 1998) as well as the GABAA receptor (Orchinik et al., 2001), in the stress relevant brain regions with the most pronounced increase found in the basket cells (Stone et al., 2001). Moreover, a recent report from our group demonstrates that chronic psychosocial stress reduces the number of hippocampal PV interneurons, a subtype of GABAergic interneurons, in the dentate gyrus and the CA3 area of treeshrews (Czéh B, et al., 2005). These results suggest a modulated GABA synthesis and GABAergic transmission following chronic stress. Parallel to these findings, accumulating evidences from 
clinical studies suggest a dysregulation of the GABAergic system in depressed patients (Brambilla et al., 2003; Hasler et al., 2007; Krystal et al., 2002; Sanacora et al., 1999) and the GABAergic signaling has been proposed as potential therapeutic target for novel antidepressants (Krystal et al., 2002).

Moreover, it is noteworthy that the impact of chronic stress on the GABAergic transmission can also be indirect. In the cerebral cortex, CB1 receptors, which mediate the signaling of endocannabinoids, are expressed on the axon terminals of some specific subtype of GABAergic interneurons such as the CCK expressing basket cells in the hippocampus (Galarreta et al., 2008; Hájos et al., 2000). As mentioned above, endocannabinoids have been identified as an important retrograde neurotransmitter which predisposes CB1 expressing interneurons to modulation of GABA release depending on activity of target postsynaptic cell (Maejima et al., 2001; Yoshida et al., 2002; Freund et al., 2003; Reich et al., 2005). Recently, increasing evidences suggest that endocannabinoid transmission in the brain is an important modulator of the stress response (Fride et al. 2005; Steiner et al. 2008). Chronic stress differentially alters endocannabinoid content (Hill et al., 2005; Patel et al., 2005; Rademacher et al., 2008) as well as CB1 receptor expression (Hill et al., 2005) and binding activity (Hill et al., 2009) in distinct limbic brain areas. Physiological studies have also demonstrated that $\mathrm{CB} 1$ receptor mediated modulation of GABAergic transmission is changed following chronic stress in striatum and amygdala (Rossi et al., 2008; Patel et al., 2009). In parallel, genetic variations in the CB1 gene CNR1 in humans have been reported to be associated with vulnerability to stressful life events and with the development of depressive symptoms (Juhasz et al., 2009).

However, despite the pivotal role of the interneurons in the neuronal network activity, the physiological aspect of the chronic-stress-induced changes in the GABAergic system as well as the relevant endocannabinoid signaling and how the pathophysiology contributes to behavioral effects of stress are still substantially under-investigated.

\subsection{The chronic restraint stress paradigm}

Restraint stress of various durations is a paradigm that has been extensively used in studies 
investigating changes in neural morphology, neurophysiology which may underlie stress-induced behavior deficits (Magariños and McEwen, 1995; Watanabe et al., 1992; Radley et al 2004). Compared to controls, chronically restrained animals exhibit reduced body weight gain and increased relative adrenal weights both of which are reliable physiological indicators for repetitive stress (Magariños and McEwen, 1995; McLaughlin, et al 2007). The physiological effects of chronic stress resulted from prolonged elevated secretion of corticosterone which leads to a shift in energy metabolism favoring fat loss from the adipose stores (Gomez et al., 2002). The behavioral effects of chronic restraint stress include compromised hippocampus dependent cognitive functional like spatial memory (Luine et al., 1994; Conrad et al., 1996), enhanced fear conditioning as well as anxiety (Conrad et al., 1999) and increased depressive-like behavior (Marais et al., 2009)

\subsection{Aims of the thesis}

The aims of the present thesis were to investigate effects of acute as well as chronic restraint stress on activities of interneurons in the hippocampal formation and the mPFC and to identify cellular pathways which may either directly contribute or provide potential pathogenetic mechanisms to observed effects. In order to address this general objective, the following experiments were designed and conducted:

1. Whole cell patch-clamp recordings were used to examine how hippocampal GABAergic transmission was altered by $30 \mathrm{~min}$ of acute restraint. Because acute stress increases the level of circulating corticosterone, effects of the potent synthetic GR agonist dexamethasone (DEX) on GABAergic transmission were tested in vitro and GR signaling pathway was investigated through pharmacological manipulations.

2. Whole cell patch-clamp recordings were used to examine effects of chronic restraint stress on hippocampal GABAergic transmission. In addition, the functional dichotomy of PV and CCK interneurons in the context of chronic stress was studied, specifically the differential effects of chronic stress on PV and CCK interneurons with regard to their ability to generate rhythmic spontaneous IPSCs. Effects of chronic restraint stress on the number of hippocampal PV- and CCK-immunoreactive interneuron were also examined. 
Moreover, physiological aspect of the chronic-stress-induced regulation of the endocannabinoid signaling in the hippocampus was investigated using whole cell patch-clamp.

3. Using the optical fractionator technique, chronic stress effects on the total number of PVimmunoreactive neurons was quantified in the left and right mPFC.

4. Using whole cell patch-clamping, effects of 30 min acute stress were investigated again in the left and right mPFC. Because the mesocortical dopaminergic pathway is implicated in the hemispheric asymmetry of mPFC functioning in response to stress, effects of dopamine and D1 receptor antagonists were examined. 


\section{Materials and Methods}

\subsection{Experimental animals}

Adult male Sprague Dawley rats (Harlan Winkelmann, Borchen, Germany) were used for all the experiments in the present thesis (control: $n=57$; acute-stress: $n=13$; chronic stress: $\mathrm{n}=21$ ). Animals were kept under an inverse light cycle (light off from 7:00 AM to 7:00 PM). The experiments were performed in accordance with the European Communities Council Directive of November 24, 1986 (86/EEC) and the US National Institutes of Health Guide for the Care and Use of Laboratory Animals, and were approved by the Lower Saxony Federal State Office for Consumer Protection and Food Safety, Germany.

\subsection{Restraint stress}

Experimental animals weighing $170-200 \mathrm{~g}$ at the beginning of the experiment, were group housed (3-4 animals / cage) and maintained under controlled temperature between $18^{\circ} \mathrm{C}$ and $22^{\circ} \mathrm{C}$. Animals were randomly assigned to different groups and allowed to habituate to the housing conditions and daily handling for at least 10 days before the onset of any experiment. Animals of the acute-stress group were restrained for $30 \mathrm{~min}$ in well-ventilated polypropylene tubes without access to food and water and were decapitated immediately thereafter. During restraint, animals were not physically compressed and did not experience pain. Animals of the chronic stress group were restrained daily for six hours (from 8:00 to $14: 00$ which is during their active period) for a total of 21 days (McLaughlin et al., 2007). Food was also withheld from the controls during the restraint period to ensure that effects on body weight gain were not simply a result of limited food availability. Throughout the entire experiment, body weight was recorded daily prior to the onset of restraint. All experimentation including daily weighing and handling of controls was performed under dimmed red light. All animals in the chronic stress experiment were sacrificed $24 \mathrm{hrs}$ following the last stress exposure. Increased adrenal weight is an indicator of sustained stress (Magariños and McEwen, 1995; McLaughlin et al., 2007). Therefore, in the experiments where brains were perfused for 
immunocytochemistry, adrenal glands were removed immediately after perfusion and weighed. Adrenal weight was expressed as milligrams per gram of body weight measured on the last experimental day.

\subsection{Electrophysiology}

\subsubsection{Brain slice preparation}

The preparation of coronal brain slices containing either dorsal hippocampus or mPFC were conducted following the general procedure of generating thin slices from the tissue of rat central nervous system described in Karson et al. (2008) and Liu \& Aghajanian (2008), respectively. Rats were decapitated using a guillotine. The whole brain was then rapidly and carefully removed from the cut-open skull and immediately transferred into ice-cold artificial cerebrospinal fluid (ACSF), which was already bubbled with $95 \% \mathrm{O}_{2}$ and $5 \% \mathrm{CO}_{2}$. The ASCF solution for cutting in all experiments consisted of $1 \mathrm{MgCl}_{2}, 2.5 \mathrm{KCl}, 2 \mathrm{MgSO}_{4}, 1.25$ $\mathrm{Na}_{2} \mathrm{HPO}_{4}, 26 \mathrm{NaHCO}_{3}, 14$ Glucose, 198 Sucrose, $1.5 \mathrm{CaCl}_{2}, 1$ kynurenic acide and 2 ascorbic acid (in $\mathrm{mM}$ ). Acute coronal brain slices (350 $\mu \mathrm{m}$ thickness) were cut using a vibratome slicer (752M Vibroslice, Campden IInstruments, UK). After sectioning, each slice was quickly placed into an incubation chamber containing aerated recording ACSF (extracellular solution). Slices were incubated at $30^{\circ} \mathrm{C}$ for $1 \mathrm{hr}$ and at room temperature for at least $30 \mathrm{~min}$ before starting the recordings. The extracellular solution in all experiments consisted of $125 \mathrm{NaCl}, 2.5 \mathrm{KCl}, 1.25 \mathrm{Na}_{2} \mathrm{HPO}_{4}, 2 \mathrm{MgSO}_{4}, 26 \mathrm{NaHCO}_{3}, 1.5 \mathrm{CaCl}_{2}, 1$ ascorbic acid, 14 glucose (in $\mathrm{mM})\left(\mathrm{pH} 7.4\right.$, aerated with $\left.95 \% \mathrm{O}_{2}-5 \% \mathrm{CO}_{2}\right)$.

\subsubsection{Whole-cell patch-clamping}

Whole-cell voltage-clamp recordings were made in acute coronal brain slices either from CA1 pyramidal neurons of the dorsal hippocampus or from layer II/III pyramidal neurons of the right as well as left mPFC. During the recordings, the slices were placed into the glass bottomed recording chamber and fixed by platinum wire with a grid of parallel nylon threads to avoid dislocation of slices. The slice was continuously perfused with aerated extracellular solution during the whole recording using a water pump (Watson Marlow, USA).The brain 
slices were visualized under an Axioscope 2 FSplus microscope (Zeiss, Germany) using a $5 x$ objective to locate brain regions of interest and a $40 x$ water immersion objective to locate neurons for recording. All experiments were performed in the voltage-clamp configuration. To achieve the whole-cell mode, first a giga-seal was formed by touching the cell surface with pipette and applying gentle suction. Then a short pulse of negative pressure was applied to the electrode, which ruptured the patch of neuronal membrane under the pipette. The whole-cell configuration was thus established. Afterwards, the recordings were performed at a holding potential of $-70 \mathrm{mV}$. Patch clamp recordings were performed using a WPC-100 amplifier (ESF, Göttingen). Patches with a serial resistance of $>20 \mathrm{M} \Omega$, a membrane resistance of $<0.8 \mathrm{G} \Omega$, or leak currrents of $>150 \mathrm{pA}$ were excluded. The membrane currents were filtered by a four-pole Bessel filter at a corner frequency of $2 \mathrm{kHz}$, and digitized at a sampling rate of $5 \mathrm{kHz}$ using the DigiData 1322A interface (Axon Instruments/Molecular Devices, Sunnyvale, CA, USA). All experiments were conducted at room temperature. The pipettes used for recording were pulled from borosilicate glass micropipettes (GC 150-10F, Clark Electromedical Instruments, UK) using a multistage puller (P87, Sutter Instrument Co. Novato, USA). Resistance of the electrodes varied between 4 and $8 \mathrm{M} \Omega$.

\subsubsection{Spontaneous and miniature IPSC recordings}

The pipette solution for all the spontaneous and miniature recordings contained $140 \mathrm{KCl}, 1$ $\mathrm{CaCl}_{2}, 10 \mathrm{EGTA}, 2 \mathrm{MgCl}_{2}, 0.5 \mathrm{Na}_{2}$-GTP, $4 \mathrm{Na}_{2}$-ATP, 10 HEPES (in mM); pH was adjusted to 7.2 with $\mathrm{KOH}$. Spontaneous GABAergic inhibitory postsynaptic currents (sIPSCs) were recorded at a holding potential of $-70 \mathrm{mV}$ in the presence of $10 \mu \mathrm{M}$ AMPA antagonist 6-cyano-7-nitroquinoxaline-2,3-dione $\quad(\mathrm{CNQX}), \quad 40 \mu \mathrm{M} \quad$ NMDA antagonist 2-amino-5-phosphonovaleric acid (APV) and $1 \mu \mathrm{M}$ glycine receptor antagonist strychnine. Miniature IPSCs (mIPSCs) were recorded with the same drugs, but in addition in the presence of $0.5 \mu \mathrm{M}$ tetrodotoxin (TTX) to block voltage-gated sodium channels thereby the generation of action potentials. Signals with amplitudes at least twofold above the background noise were analyzed. There were no significant differences in noise levels between control and stress animals. 
To observe the influence of intracellular $\left[\mathrm{Ca}^{2+}\right]$ on the effect of chronic stress, the slices were incubated in the cell permeable $\mathrm{Ca}^{2+}$ chelator EGTA-AM $(100 \mu \mathrm{M}$ in the recording solution) at room temperature. EGTA-AM was washed off after 30 minutes in incubation.

\subsubsection{Depolarization induced suppression of inhibition - DSI}

The endocannabinoid signaling was assessed using DSI test established by Ohno-Shosaku et al. (2001). DSI was initiated by a single $5 \mathrm{~s}$-depolarizing voltage step from $-70 \mathrm{mV}$ to $0 \mathrm{mV}$ in the hippocampus and a $10 \mathrm{~s}$ voltage step in the mPFC. All DSI tests were conducted at least $3 \mathrm{~min}$ after achieving a stable whole-cell configuration. Three DSI tests were applied to each cell, with a 1 min-interval between each test. The effect of DSI was calculated as the ratio of sIPSC frequency after depolarization to that before depolarization. Normalized data were then used for further statistical analysis. To illustrate the time course of DSI, the average of the normalized sIPSC frequency of 10-s epochs of recordings from hippocampus and 5-s epochs from mPFC were plotted against time after depolarization.

\subsubsection{Data analysis}

Data acquisition was performed using commercially available software (pClamp 10.1; Axon Instruments/Molecular Devices, Sunnyvale, CA, USA). MiniAnalysis 6.0.9 (Synaptosoft Inc., Decatur, GA, USA) was used to perform amplitude and frequency analysis of sIPSCs and mIPSCs.

For analysis of theta rhythms, power spectrum and autocorrelations were performed in Clampfit 10.1. We calculated a value of "relative theta power" for each cell by summing the spectral power between 4 and $14 \mathrm{~Hz}$, and dividing this by the total spectral power between 1 and $50 \mathrm{~Hz}$ during $10 \mathrm{~s}$ of sIPSC activity (Karson et al., 2008).

\subsubsection{Drugs for electrophysiology}

CCK-8, LY225910 were obtained from Tocris. The dexamethasone-BSA conjugate was obtained from Steraloids Inc. (Newport, RI, USA). EGTA-AM was obtained from Molecular Probe (Invitrogen, Carlsbad, CA, USA). All other chemicals were obtained from 
Sigma-Aldrich (Sigma-Aldrich, St Louis, MO, USA).

\subsection{Perfusion and brain dissection}

Control and chronically stressed rats ( $n=14$ / group) were terminally anesthetized with an overdose of ketamine $(50 \mathrm{mg} / \mathrm{ml})$, xylazine $(10 \mathrm{mg} / \mathrm{ml})$ and atropine $(0.1 \mathrm{mg} / \mathrm{ml})$, and perfused transcardially with ice-cold $0.9 \%$ saline, followed by $200 \mathrm{ml}$ of ice-cold fixative containing $4 \%$ paraformaldehyde in $0.1 \mathrm{M}$ sodium-phosphate buffer (PBS, pH 7.2) for $15 \mathrm{~min}$. The descending aorta was clamped so that adrenal glands were not perfused. To prevent post-perfusion artifacts in the brains, the heads were post-fixed over night in fresh fixative at $4^{\circ} \mathrm{C}$. On the following day, the brains were gently removed, washed thoroughly in PBS and immersed in $30 \%$ sucrose in PBS at $4^{\circ} \mathrm{C}$ for $24 \mathrm{hr}$ for cryoprotection. After calibration, brains were frozen, and coronal sections $(50 \mu \mathrm{m})$ were cut in a cryostat.

\subsection{Immunocytochemistry}

Samples from the two groups were processed in parallel to avoid any nonspecific effect of the staining procedure. First, sections were washed in PBS and then treated with $1 \% \mathrm{H}_{2} \mathrm{O}_{2}$ in PBS for $30 \mathrm{~min}$ at room temperature in order to quench endogenous peroxidase activity. Sections were then rinsed three times in PBS and blocked for $1 \mathrm{hr}$ at room temperature in PBS containing $3 \%$ normal goat-serum and $0.5 \%$ Triton $X-100$. Following blocking, sections were incubated either with a polyclonal rabbit anti-cholecystokinin-8 antibody (AbCam, Cambridge, MA, USA, AB43842, dilution 1:10000), or with monoclonal mouse anti-parvalbumin antibody (Chemicon/Millipore, Temecula, CA, USA; MAB1572, dilution 1:3000) overnight at $4{ }^{\circ} \mathrm{C}$. The next day, sections were rinsed three times in PBS and then incubated for $2 \mathrm{hr}$ at room temperature either with biotinylated goat anti-rabbit (diluted 1:200; Vector Laboratories, Burlingame, CA, USA), or with biotinylated goat anti-mouse (diluted 1:200, Vector Laboratories) secondary antibody. After washing, the sections were incubated with avidin-biotin-horseradish peroxidase complex (Vectastain Elite ABC Kit, Vector Laboratories) for $1 \mathrm{~h}$, rinsed again in PBS, developed for $5 \mathrm{~min}$ in diaminobenzidine (DAB Peroxidase Substrate Kit, Vector Laboratories), and then thoroughly rinsed in PBS. The 
sections were mounted on glass slides in a $0.1 \%$ gelatin solution and dried overnight, after which they were cleared in xylene for 30 min and coverslipped with Eukitt (Kindler, Freiburg, Germany).

\subsection{Quantification of CCK and PV-immunoreactive neurons in the hippocampus}

Every sixth section throughout the dorsal hippocampus was examined yielding a mean of 6 sections per animal. The brain sections were imaged with a Zeiss III RS microscope. Cell counting was conducted using the Neurolucida 7.53.3 software (Microbrightfield, Colchester, VT, USA). A $6.3 \times$ objective (NA 0.16) was used to outline the borders of the regions of interest and a $16 \times$ objective (NA 0.16) was used to label cells. Cell numbers are expressed as densities, i.e. the number of counted cells was divided by the volume of the analyzed hippocampal area which was calculated automatically by the software.

\subsection{Stereology - Quantification of PV-immunoreactive neurons in the mPFC}

The stereological techniques are a new generation of quantitative methods that were developed since 1980s. Compared to traditional assumption-based neuron counting methods, the new designed-based three dimensional stereological counting methods require no assumption about size, shape and orientation of the structure of interest as well as the neurons to be quantified. Any methodical bias, which will always yield biases that will in turn have unpredictable effects on the results, can therefore be eliminated.

In my study, numbers of PV-immunoreactive neurons in the mPFC were estimated with the modified optical fractionator (West et al, 1991; West, 1999; Czéh et al., 2005). It is a stereological technique that combines the optical dissector (Sterio, 1984) with the fractionator sampling scheme (Gundersen et al, 1988). The detailed processes of stereological neuron counting have been documented previously (West and Gunderson, 1990; Cruz-Orive and Weibel, 1990; West et al., 1991; West, 1999; Keuker et al., 2001) and briefly described as follows:

The optimal parameters of the fractionator sampling scheme were predetermined in a pilot experiment, and were uniformly applied to all the animals. In my experiment, the rat forebrain 
was serially sectioned and for quantification, every 3rd coronal section was systematically sampled, yielding an average of 17 sections per animal. The first section from each animal that was sampled for analyzing was always randomly chosen from the number one to six.

The brain sections were imaged with a Zeiss III RS microscope. Cell counting was conducted using the Stereoinvestigator 7.53.3 software (Microbrightfield, Colchester, VT, USA). The details concerning the setup were previously described by Keuker et al (2001). A 6.3x objective (NA 0.16) was used to outline the border of the region of interest and a $16 \times$ objective (NA 0.16) was used to count cells.

An optical dissector is an imaginary cube placed into a section and is used as a three dimensional probe for counting. The $x-y$ plane of the optical dissector is the counting frame. During the examination, the software generated a two-dimensional image of the optical dissector, representing the counting frame with the left and bottom lines designated as the exclusion sides. In addition, an imaginary mesh grid, representing the sampling region, was also created by the software and was randomly superimposed onto the section. Each optical dissector was placed systematically in each low left corner of the mesh grid.

Neurons which are fully located within each optical dissector should be counted; neurons which are partially located within each optical dissector but in any way do not touch the extended exclusion sides or the top plane of the optical dissector should also be counted. The area of the counting frame, $a$ (frame), and the sampling region, $A(x, y, s t e p)$, were selected such that the average number of neurons counted per optical dissector should be 1-2. After having counted all the immunoreactive cells fulfilling the criteria of sampling $(\Sigma Q)$, the total number of cells was estimated using the following formula (West and Gunderson, 1990): $N_{\text {total }}=\sum Q \times \frac{1}{s s f} \times \frac{1}{a s f} \times \frac{1}{t s f}($ Equation 1$)$

The section sampling fraction (ssf) represents the proportion of sections sampled for examination. The value is $1 / 3$ in my study because every third sections of the forebrain were selected. The area sampling fraction (asf) is defined by the ratio of the area of the counting frame and the area of the sampling region: $a(f r a m e) / A(x, y, s t e p)$. In my experiment, the size of the dissector frame area, a(frame) was $150 \mu \mathrm{m} \times 130 \mu \mathrm{m}$, and the sampling area, $A(x, y$ step) was $400 \mu \mathrm{m} \times 450 \mu \mathrm{m}$, yielding asf equals $13 / 120$. The thickness sampling fraction (tsf) is the 
ratio of the height of optical dissector and the thickness of sections. Here, since we applied the modified optical dissector method without using guard zones at the upper and lower surfaces of the section, the height of the optical dissector equals the section thickness (Czéh et al., 2005). Therefore tsf equals 1.

Quantification was carried out in the mPFC without discrimination between subregions.

The precision of an individual neuron number estimation is expressed by the coefficient of error, $\mathrm{CE}$, which captures the intra-individual variation due to stereological estimating procedure. The individual CE is calculated with the following formula modified by Keuker et al (2001):

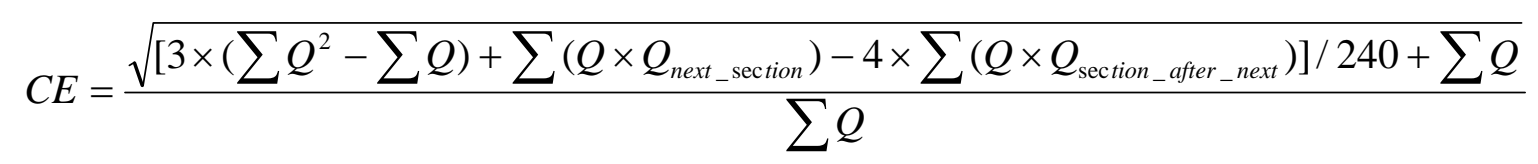

(Equation 2). For an optimal sampling scheme, the coefficient of variance of the estimations, expressed by the mean $\mathrm{CE}^{2}$, should make the minor contribution to the observed group coefficient of variance $\mathrm{CV}^{2}$ ( $\mathrm{CV}=$ standard deviation / mean $)$ and be less than the true biological coefficient of variance $\left(B C V^{2}\right)$. Regarding the equation $C V^{2}=B C V^{2}+C E^{2}$ (Equation 3 ), the $C E^{2}$ should thus be less than $50 \%$ of the $C V^{2}$ for an efficient estimate of the total neuron number within the structure of interest.

\subsection{Statistical analysis}

Analysis was made using Prism5 (GraphPad, San Diego, CA, USA). Data are presented as mean $\pm S E M$. Different statistic tests, such as unpaired/paired sample Student's t-test, one-way/two-way ANOVA, were used as appropriate and specified during result presentation. Effects were considered statistically significant at $P<0.05$. 


\section{Results}

3.1 Part I. The effects of GR stimulation and stress in the hippocampus

\subsubsection{Effects of the GR agonist dexamethasone on GABAergic transmission}

\subsubsection{Facilitation of hippocampal GABAergic transmission by the glucocorticoid dexamethasone}

It is well known that stress affects the function of hippocampal excitatory transmission via glucocorticoid action. Here we hypothesize that GR activation may also affect the inhibitory network. To directly test this, the potent and selective GR agonist DEX was applied on hippocampal slices of rats. After the application of DEX (25nM), we detected a rapid increase in SIPSC frequency $\left(6.0 \pm 0.6 \mathrm{~Hz}\right.$ before and $9.2 \pm 0.7 \mathrm{~Hz}$ after $\mathrm{DEX}, t_{8}=8.386, P<0.0001$, paired t-test; Fig. $1 \mathrm{~A}, \mathrm{C} 1)$ and amplitude $(98.9 \pm 14.4 \mathrm{pA}$ before and $140.8 \pm 25.5 \mathrm{pA}$ after DEX, $t_{8}=3.084, P=0.015$, paired $t$-test; Fig. $1 \mathrm{~A}, \mathrm{C} 2$ ). In all experiments, the initial significant facilitating effect occurred $5 \mathrm{~min}$ after exposure to DEX. This effect accelerated for a few minutes and subsided gradually afterwards (Fig. 1B1,B2, one-way ANOVA followed by post hoc Dunnett's Multiple Comparison). In addition, in 6 of the 9 cells recorded, DEX caused burst-like activities which were not detected before DEX application (Fig. 1A, arrow in the lower trace). Such a fast DEX effect has not been described before (Maggio and Segal, 2009). By contrast, miniature IPSCs (mIPSCs) showed no significant changes in response to DEX application (frequency: $2.3 \pm 0.3 \mathrm{~Hz}$ before and $2.2 \pm 0.3 \mathrm{~Hz}$ after DEX, $t_{6}=0.6281$, n.s; amplitude: $52.3 \pm 2.8 \mathrm{pA}$ before and $54.0 \pm 2.3 \mathrm{pA}$ after $\mathrm{DEX}, t_{6}=0.3932$, n.s, paired t-test; Fig. 1D1,D2), indicating that the rapid effect of DEX which was observed here does not act on the terminals. 


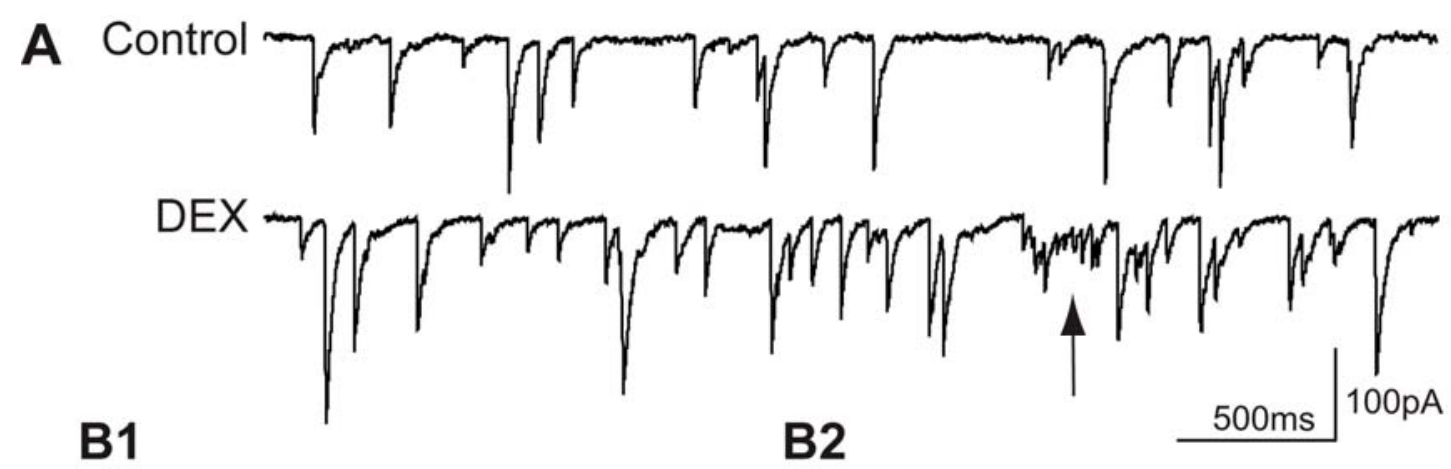

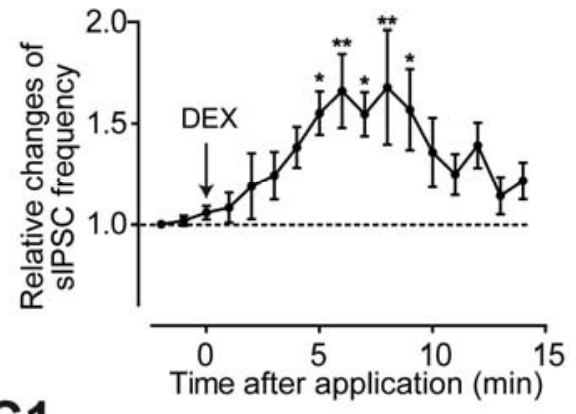

C1

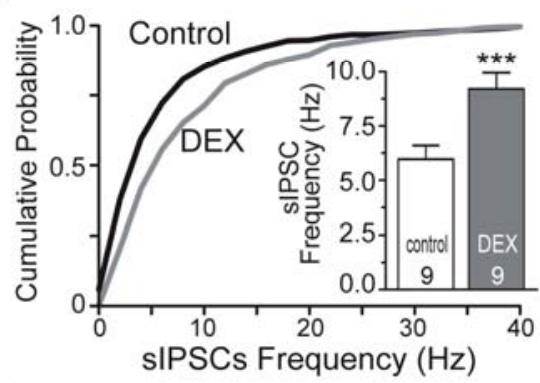

C2

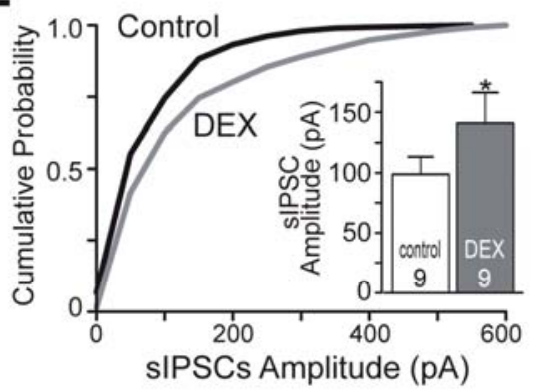

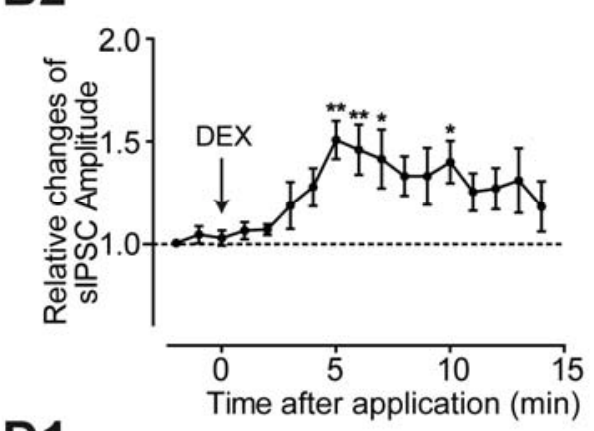

D1

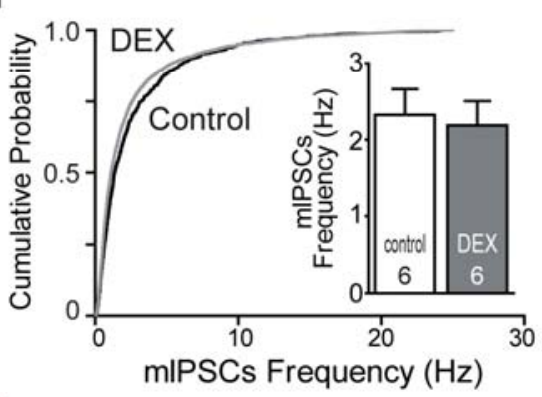

D2

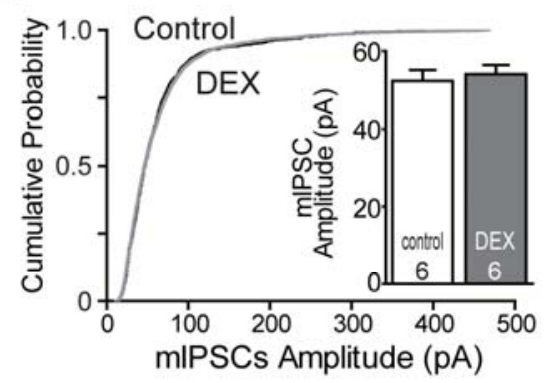

Figure 1. Glucocorticoid receptor stimulation facilitates GABAergic transmission. A: Representative recordings of sIPSCs in a CA1 pyramidal cell before and $\sim 7 \mathrm{~min}$ after the application of the GR agonist DEX which rapidly increases both frequency and amplitude of sIPSCs. B: Time course of DEX effect on sIPSC frequency (B1) and amplitude (B2) (1-min bins; graphs show relative changes). C: Numerical data of the means and cumulative plot of frequency $(\mathrm{C} 1)$ and amplitude (C2) of sIPSCs before (white bars) and 5-9 min after DEX (gray bars). D: Numerical data of the means and cumulative plot of frequency (C1) and amplitude (C2) of mIPSCs before (white bars) and 5-9min after DEX (gray bars). Data are means \pm SEM. Numbers in the bars indicate the number of neurons tested for each experimental condition, same cells in all conditions. Six normal adult male rats were used; ${ }^{\star} \mathrm{P}<0.05,{ }^{\star \star} \mathrm{P}<0.01,{ }^{\star \star \star} \mathrm{P}<0.001$. Modified from Hu et al., 2010. 


\subsubsection{Dexamethasone effect mediated through a rapid non-classical GR mechanism}

The rapid onset of the stimulatory effect of DEX on GABA transmission was quite unexpected. Although it has been reported that the activity of the hippocampal pyramidal neurons can be promoted via a nongenomic corticosterone action (Karst et al., 2005b), the observed stimulation of sIPSCs could not be due to an increase in the excitatory drive because all the glutamatergic transmission was blocked in the presence of CNQX and APV (antagonists for AMPA and NMDA receptors, respectively) to isolate sIPSCs. Therefore we performed a series of experiments to examine the cellular target of DEX. First, the above described DEX effect remained in the presence of the nuclear MR and GR antagonists spironolactone $(10 \mu \mathrm{M})$ (frequency: $3.9 \pm 0.6 \mathrm{~Hz}$ before and 7.0 $\pm 1.1 \mathrm{~Hz}$ after DEX, $t_{6}=4.854, P=0.0028$; amplitude: 64.3 $\pm 4.6 \mathrm{pA}$ before and $89.1 \pm 12.1 \mathrm{pA}$ after $\mathrm{DEX}, t_{6}=2.560, P=0.043$, paired t-test; Fig. $2 \mathrm{~A}$ ) and mifepristone $\left(10 \mu \mathrm{M}\right.$; frequency: $5.5 \pm 0.8 \mathrm{~Hz}$ before and $8.2 \pm 1.4 \mathrm{~Hz}$ after DEX, $t_{5}=3.758$, $P=0.013$; amplitude: $73.6 \pm 11.8 \mathrm{pA}$ before and 103.5 $\pm 21.6 \mathrm{pA}$ after $\mathrm{DEX}, t_{5}=2.770, P=0.039$; paired t-test; Fig. 2A), respectively. The antagonists by themselves showed no significant effects on the SIPSCs. Second, bath application of the membrane impermeable BSA-DEX conjugate $(250 \mathrm{nM})$ retained the stimulatory effect of DEX on GABA release (frequency: 6.4 $\pm 0.5 \mathrm{~Hz}$ before and $10.0 \pm 0.5 \mathrm{~Hz}$ after BSA-DEX, $t_{6}=16.59, P<0.0001$; amplitude: $78.5 \pm 6.2$ pA before and 101.0 $\pm 8.0 \mathrm{pA}$ after BSA-DEX, $t_{6}=4.654, P=0.0035$; paired t-test; Fig. $2 \mathrm{~B}$ ). Finally, intracellular application of the G-protein inhibitor GDP- $\beta-S(0.5 \mathrm{mM})$ via the pipette solution blocked the DEX-induced increase in SIPSC frequency $(6.9 \pm 0.5 \mathrm{~Hz}$ before and 6.3 $\pm 0.4 \mathrm{~Hz}$ after DEX, $t_{5}=1.728$, n.s; Fig. $2 \mathrm{C}$ ). Taken together, our data strongly suggest that the rapid effect of DEX (5 $\mathrm{min}$ after the application) was mediated by a nongenomic membrane bound GR which activated a G protein dependent signaling pathway. This fast mechanism differs from genomic GR mediated processes ( $>25 \mathrm{~min}$, Maggio and Segal, 2009). Furthermore, since the GDP- $\beta-S$ application was restricted to the postsynaptic pyramidal neuron, this result suggests that DEX, at least partially, acts on the postsynaptic cell and that retrograde messenger(s) mediate the stimulatory effect on GABA release. 
A1

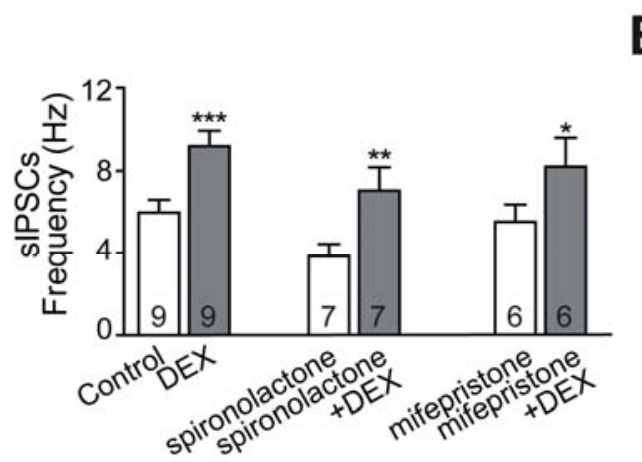

B1

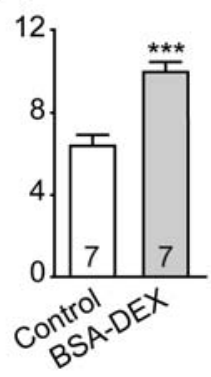

C1

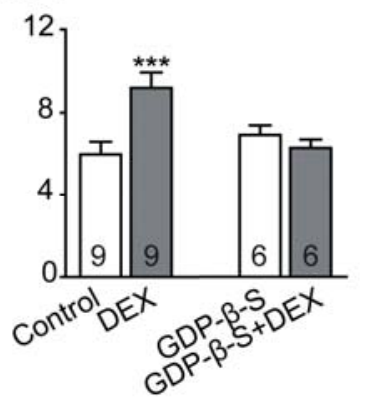

A2

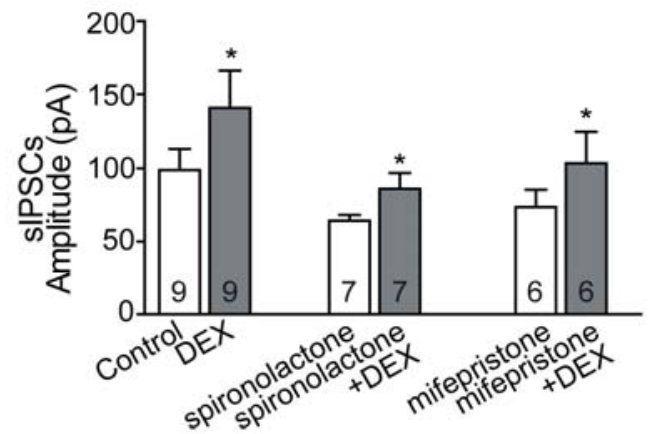

B2

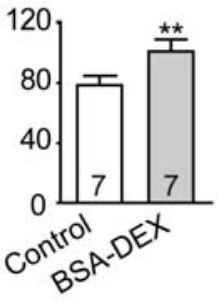

C2

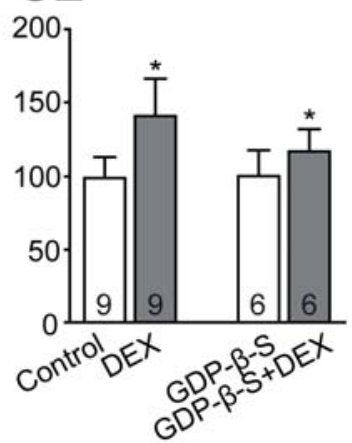

Figure 2. DEX facilitates GABAergic transmission through a nongenomic mechanism. $A 1, A 2$ : The DEX effect was resistant to the wash-in of spironolactone and mifepristone which are antagonists at nuclear MRs/GRs. B1,B2: Membrane impermeable BSA-DEX could retain the DEX effect. C1,C2: Intracellular application of GDP- $\beta-S$, which irreversibly inactivates G proteins, blocked the DEX effect. Data are means \pm SEM. Numbers in the bars indicate the number of neurons tested for each experimental condition, same cells in all conditions. Six normal adult male rats were used; ${ }^{\star} \mathrm{P}<0.05,{ }^{* *} \mathrm{P}<0.01$, ${ }^{* *} \mathrm{P}<0.001$. Modified from Hu et al., 2010.

\subsubsection{Facilitation of GABAergic transmission by dexamethasone through retrograde} nitric oxide signaling

We then examined which retrograde messenger system may be involved in the stimulatory effect of DEX on GABA release. Earlier studies have demonstrated that in the hippocampus, endocannabinoids mediate the activity dependent suppression of GABAergic inhibition via activation of CB1 receptors that are expressed specifically by CCK-expressing cells (Freund and Katona, 2003). It is very unlikely that the same retrograde CB1-mediated messenger pathway is responsible for both facilitating and suppressing GABAergic transmission. On the other hand, nitric oxide (NO)-sensitive guanylyl cyclase has been reported to be present in 
the axon terminals of both PV and CCK interneurons (Szabadits et al., 2007). We therefore focused on the retrograde NO pathway.

Intracellular application of 7-nitroindazole $(7-\mathrm{NI}, 100 \mu \mathrm{M})$, a selective inhibitor of neuronal NO synthase (NOS) which did not show any significant effect on hippocampal sIPSCs by itself, completely blocked the DEX-induced increase of sIPSC amplitude and frequency (amplitude in 7-NI: $63.1 \pm 6.6 \mathrm{pA}$; amplitude in 7-NI plus DEX: $66.2 \pm 4.6 \mathrm{pA}$; n.s.; frequency in 7-NI: 6.5 $\pm 0.3 \mathrm{~Hz}$; frequency in 7-NI plus DEX: $6.2 \pm 0.5 \mathrm{~Hz}$; n.s.; paired t-test; Fig. 3A). Similarly, a 30 min-incubation of the slices with $\mathrm{ODQ}(50 \mu \mathrm{M}$ in $\mathrm{ACSF})$, a selective inhibitor of NO-sensitive guanylyl cyclase (NOsGC), completely blocked the effect of DEX on GABA release (sIPSC amplitude in ODQ: $58.1 \pm 3.7 \mathrm{pA}$, amplitude in ODQ plus DEX: $65.7 \pm 3.1 \mathrm{pA}$, n.s.; frequency in ODQ: $6.2 \pm 0.7 \mathrm{~Hz}$, frequency in ODQ plus DEX: $6.0 \pm 0.6 \mathrm{~Hz}$, n.s.; paired t-test; Fig. 3B). Incubation with ODQ caused a small reduction in the SIPSC amplitude $\left(\mathrm{t}_{14}=2.418, P=0.0298\right.$; unpaired $\mathrm{t}$-test) but not in the frequency which is most likely due to weak activation of NOsGC at baseline level. These results demonstrate that inhibiting NO synthesis or silencing NO downstream signaling can completely block the above fast DEX effect. In addition, the NO donor SNAP (S-nitroso- $N$-acetylpenicillamine) mimicked the facilitating effect of DEX on sIPCSs. Similarly to the effect elicited by DEX, bath application of SNAP $(100 \mu \mathrm{M})$ increased sIPSC amplitude and frequency (baseline amplitude: 82.3 $\pm 4.4 \mathrm{pA}$; amplitude after SNAP $116.7 \pm 7.4 \mathrm{pA} ; P<0.005$; baseline frequency: $5.8 \pm 0.4 \mathrm{~Hz}$; frequency after SNAP 10.4 $\pm 0.8 \mathrm{~Hz} ; P<0.005$; paired t-test; Fig. 3C). Furthermore SNAP $(100 \mu \mathrm{M})$ also mimicked the time course of the fast DEX effect (c.f. Fig. 3C2/C3 to Fig. 1B1/B2). Taken together, these results indicate that the fast DEX-induced facilitating effect on GABA transmission is mediated by nitric oxide retrograde signaling. 
A 7-NI (selective inhibitor of neuronal NO synthase)
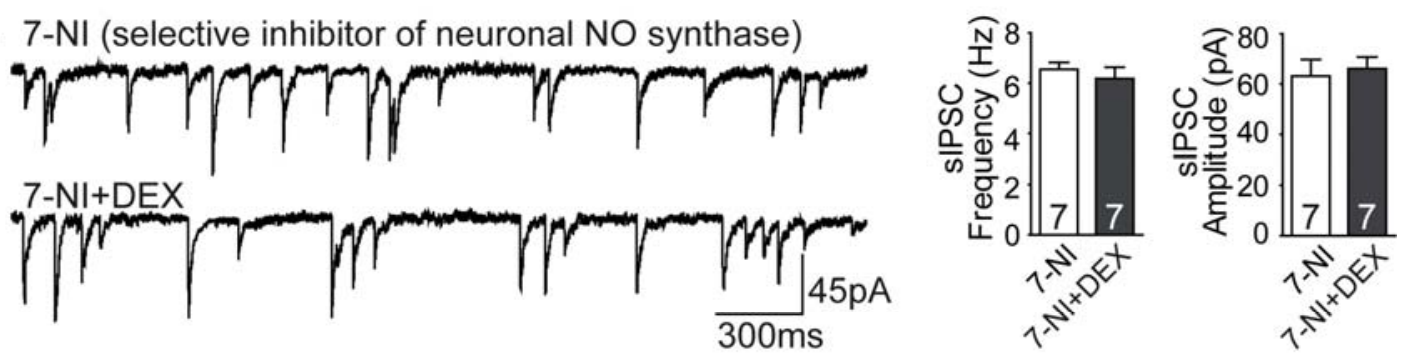

B ODQ (selective inhibitor of NO-sensitive guanylyl cyclase)
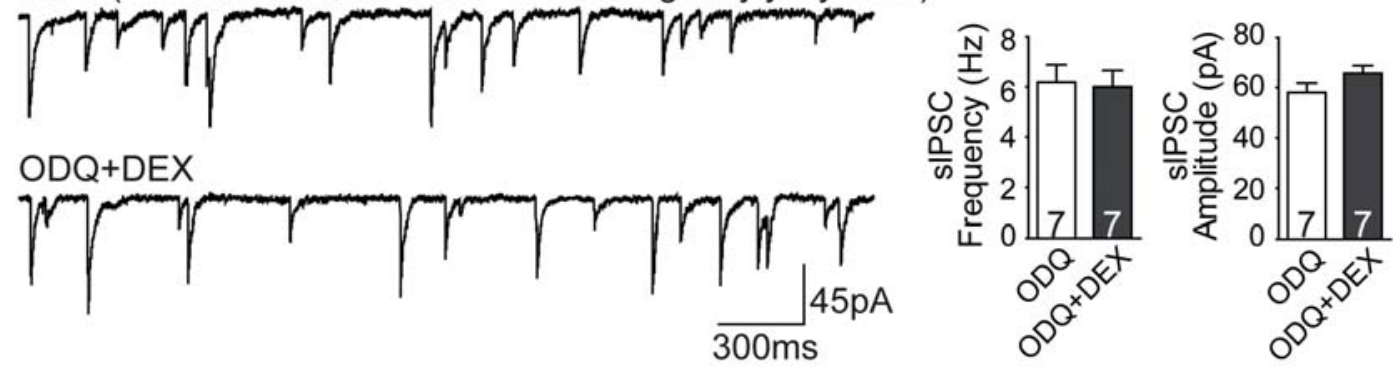

C1 Control

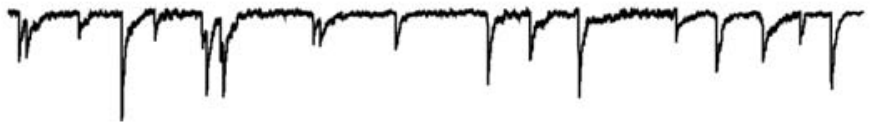

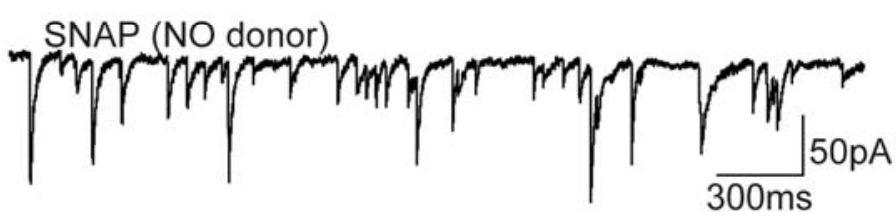

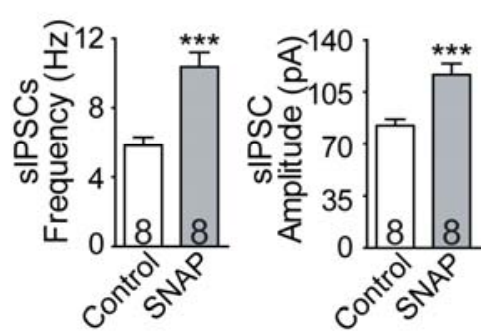

\section{C2}

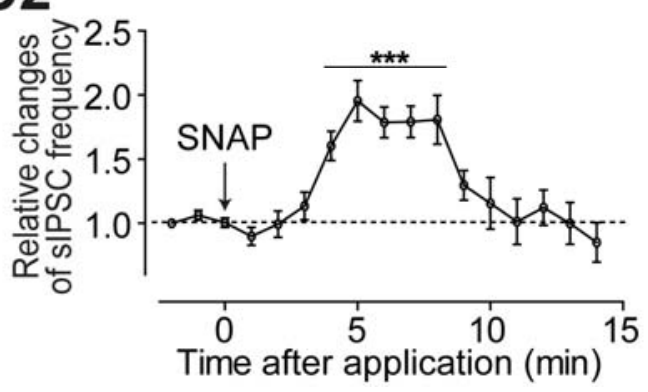

C3

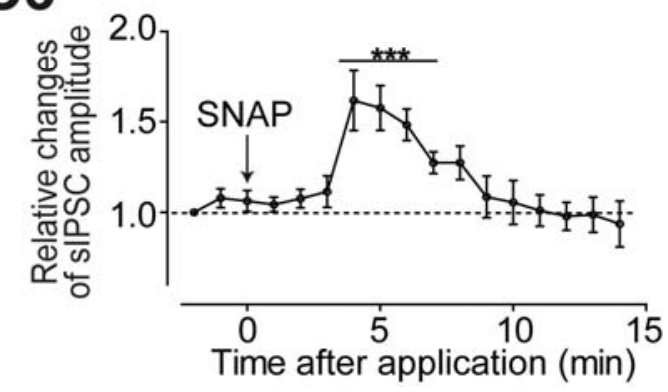


Figure 3. The DEX-induced facilitation of GABAergic transmission is mediated by retrograde nitric oxide signaling. $A$ : The $D E X$ effect is abolished in the presence of a NO synthase inhibitor 7-NI. (Left) representative recordings of sIPSCs from a CA1 pyramidal cell before and $\sim 7 \mathrm{~min}$ after DEX application, $7-\mathrm{NI}$ was applied intracellularly via the pipette solution. (Right) Numerical data of mean sIPSCs before and after DEX. B: The DEX effect is abolished in the presence of an inhibitor of NO-sensitive guanylyl cyclase, ODQ. (Left) representative recordings of sIPSCs from a CA1 pyramidal cell before and after DEX application; slices were incubated in ODQ for $\sim 30 \mathrm{~min}$. (Right) Numerical data of mean sIPSCs before and after DEX. C: Bath application of the NO donor SNAP mimicked the DEX effect. C1 left: Representative sIPSC recordings from a CA1 pyramidal cell before and $\sim 6 \mathrm{~min}$ after SNAP. C1 right: Numerical data of mean sIPSCs before and 5-9 min after SNAP. C2,C3: Time course of the SNAP effect on sIPSCs (1-min bins; Graphs show relative changes). Data are mean \pm SEM. Numbers within the bar graphs indicate the number of neurons tested for each experimental condition, same cells in all conditions; 6 normal adult male rats were used; ${ }^{* \star \star} \mathrm{P}<0.001$. Modified from Hu et al., 2010.

\subsubsection{Facilitation of GABAergic transmission by dexamethasone partially through nitric oxide-induced release of CCK}

There are substantial evidences that the neuropeptide CCK is involved in the stress response (Becker et al., 2008; Hebb et al., 2005). In addition, previous report showed that the CCK expressing basket cells are among the interneurons that express the retrograde NO signaling machinery on their terminals (Szabadits et al., 2007). Therefore it was interesting to ask, whether the enhanced firing activities in the presence of DEX would also induce CCK release from CCK interneurons which would further modulate perisomatic GABA release (Földy et al., 2007). To test this, we incubated the slices with the selective $\mathrm{CCK}_{2}$ receptor antagonist LY225910 (20 $\mu \mathrm{M}$ in ACSF) for 30min before recording. The antagonist by itself had no effect on sIPSCs, but it blocked the response to further CCK application. Then we applied 25nM DEX and observed an increase in sIPSC amplitude and frequency (amplitude in LY225910: 79.6 $\pm 9.8 \mathrm{pA}$, amplitude in LY225910 plus DEX: $89.8 \pm 7.0 \mathrm{pA}, t_{7}=2.504, P=0.04$; frequency in LY225910: $6.2 \pm 0.9 \mathrm{pA}$, frequency in LY225910 plus DEX: $7.3 \pm 0.8 \mathrm{pA}, t_{7}=4.788, P=0.002$; paired t-test; Fig. 4A,C1). However, compared to the condition without LY225910 pre-incubation, the extent of the increase was much lower (increase of amplitude: $19.5 \pm 6.0 \%$ vs. $40.8 \pm 8.8 \%$, unpaired t-test $t_{15}=1.946, P=0.07$; increase of frequency: $24.4 \pm 8.3 \%$ vs. $60.2 \pm 9.9 \%$, unpaired t-test $t_{15}=2.733, P=0.015$; Fig. $4 \mathrm{C} 2$ ). It is interesting to note that the 
duration of the fast DEX effect was shortened in the presence of LY225910 (3 min vs. 5 min; Fig. 4B) while the onset time was unaffected (one-way ANOVA with Dunnett's multiple comparison test, Fig. 4B). Furthermore, following the incubation with LY225910 the stimulatory effect of the NO donor SNAP on GABAergic transmission was also significantly reduced (increase of amplitude: $23.9 \pm 5.1 \%$ vs. $42.0 \pm 4.9 \%$, unpaired t-test, $t_{14}=2.550, P=0.02$; increase of frequency: $20.8 \pm 3.1 \%$ vs. $78.3 \pm 9.6 \%$, unpaired t-test, $t_{14}=5.718, P<0.0001$; Fig. $4 D, E)$. The fact that the $\mathrm{CCK}_{2}$ receptor antagonist partially blocked the fast effects of DEX and SNAP suggests that CCK additionally contributes to the DEX action and that NO signaling induces endogenous CCK release which in turn further facilitates GABAergic transmission.

\subsubsection{Effects of acute stress on hippocampal GABAergic transmission}

The above data demonstrate that acute application of DEX caused a rapid facilitation of GABAergic transmission in the hippocampal CA1 area through a nongenomic GR mechanism. Since stress response is mediated not only by glucocorticoids, it is important to ask whether exposure to real life stress also elicits enhancement of inhibitory transmission. To answer this, a series of experiments was performed with rats exposed to restraint stress of various durations.

First, the effect of short-term (acute) stress on hippocampal sIPSCs was investigated. In the hippocampal slices from animals exposed to $30 \mathrm{~min}$ of restraint stress, an increase in sIPSC frequency compared to the controls was found (control: $7.3 \pm 0.3 \mathrm{~Hz}$; acute stress: $11.8 \pm 0.7 \mathrm{~Hz}$; unpaired t-test $\mathrm{t}_{15}=5.424 P<0.0001$; Fig. $\left.5 \mathrm{~A}, \mathrm{~B} 1\right)$, whereas the amplitude of the sIPSCs remained unaffected (control: 90.01 $\pm 9.3 \mathrm{pA}$; acute stress: $98.41 \pm 11.2 \mathrm{pA}$; unpaired t-test $t_{15}=0.5772 P=0.5723$; Fig. 5A,B2). In addition, in 7 out of the 10 cells recorded from the acutely stressed animals, burst-like activities were frequently observed (Fig. 5A arrow), whereas in the control group, this phenomenon was sparsely detected ( 2 of the 7 cells). These data indicate that acute stress exposure results in an enhancement of hippocampal GABAergic transmission that is similar though not identical to the effect elicited by DEX (see 
Fig. 1).

A

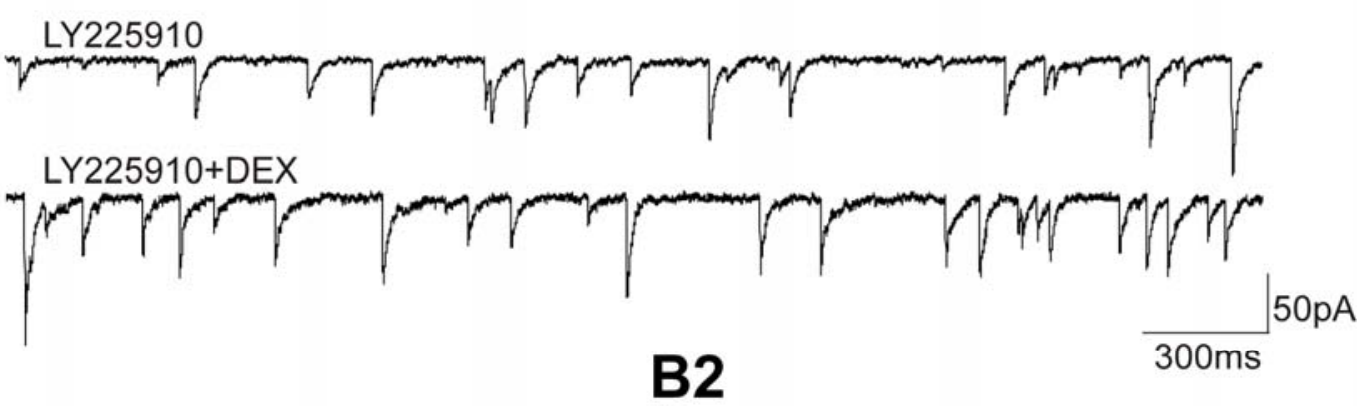

B1

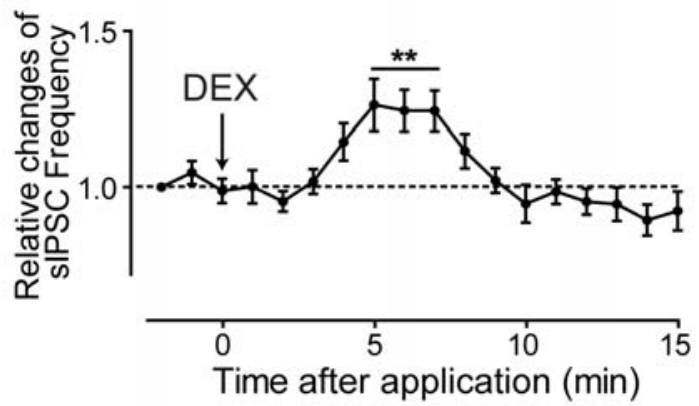

C1
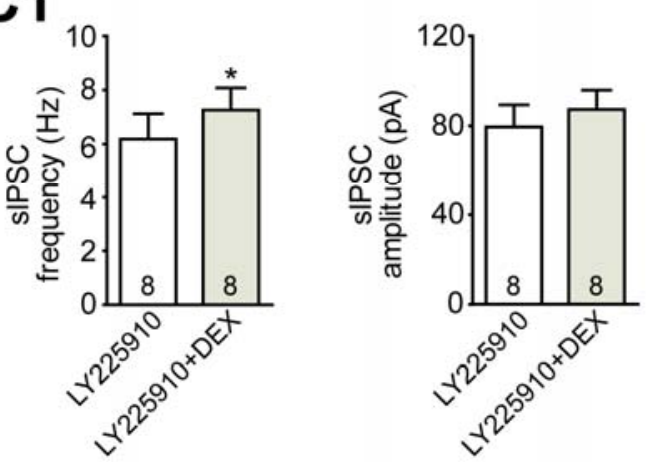

D LY225910+SNAP

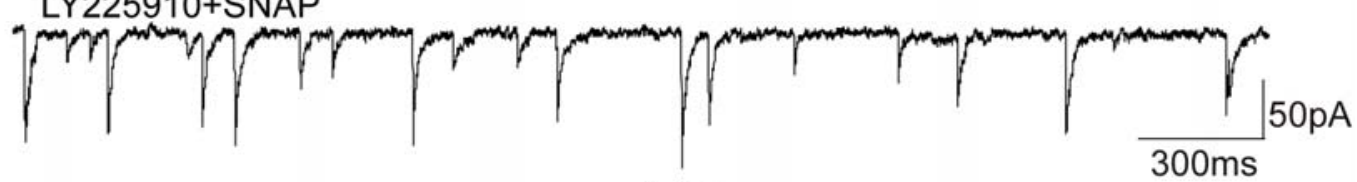

E1

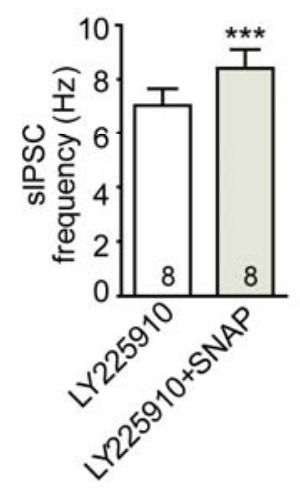

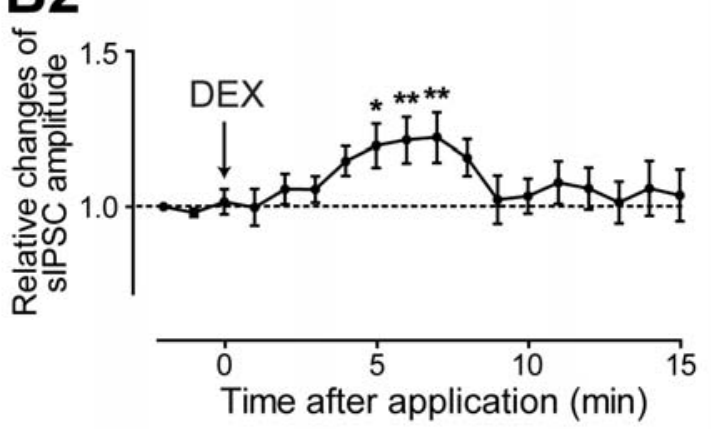

C2
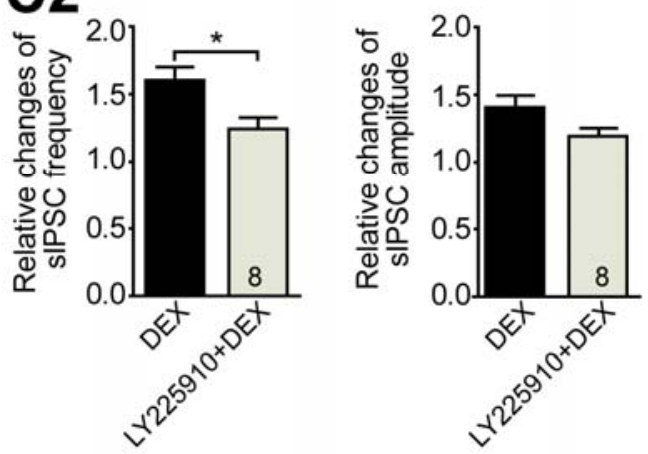

E2
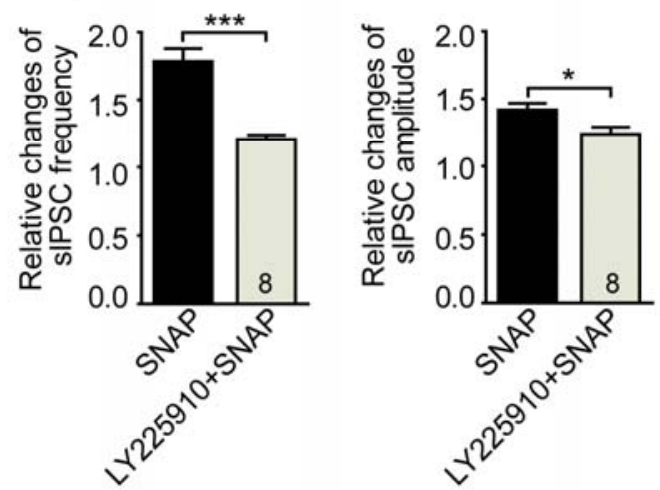
Figure 4. The DEX-induced facilitation of GABAergic transmission is partially mediated by endogenous $C C K$ release downstream from the nitric oxide signaling. $A$ : Representative sIPSCs from a CA1 pyramidal cell after incubation with the CCK2 receptor antagonist LY225910 for 30min and then $\sim 7 \mathrm{~min}$ after DEX application. B: Time course of the DEX effect following 30min-incubation with LY225910 (1-min bins; Graphs show relative changes), showing that the duration of significant DEX effect was shortened by blocking CCK2 receptor. C: Numerical data of mean sIPSCs before and 5-7 min after DEX exposure in the presence of LY225910 (C1) and mean relative changes of sIPSCs (C2) in response to DEX without and with LY225910, respectively. Bath application of DEX still induced a significant increase in SIPSCs. However, the increase was smaller compared to the condition without LY225910 preincubation, revealing that CCK mediated signaling is also involved in the DEX-induced enhancement of sIPSCs. D: Representative sIPSCs after bath application of the NO donor SNAP in the presence of LY225910. E: Numerical data of mean sIPSCs (E1) and mean relative changes of sIPSCs (E2) in response to SNAP without and with LY225910, respectively, showing that LY225910 partially blocked the stimulatory effect of SNAP. This indicates that NO signaling induces endogenous CCK release which in turn facilitates GABAergic transmission. Data are mean \pm SEM. Numbers within the bar graphs indicate the number of neurons tested for each experimental condition, same cells in all conditions; 7 normal adult male rats were used; ${ }^{\star} \mathrm{P}<0.05,{ }^{\star \star} \mathrm{P}<0.01,{ }^{\star \star \star} \mathrm{P}<0.001$. Modified from Hu et al., 2010.

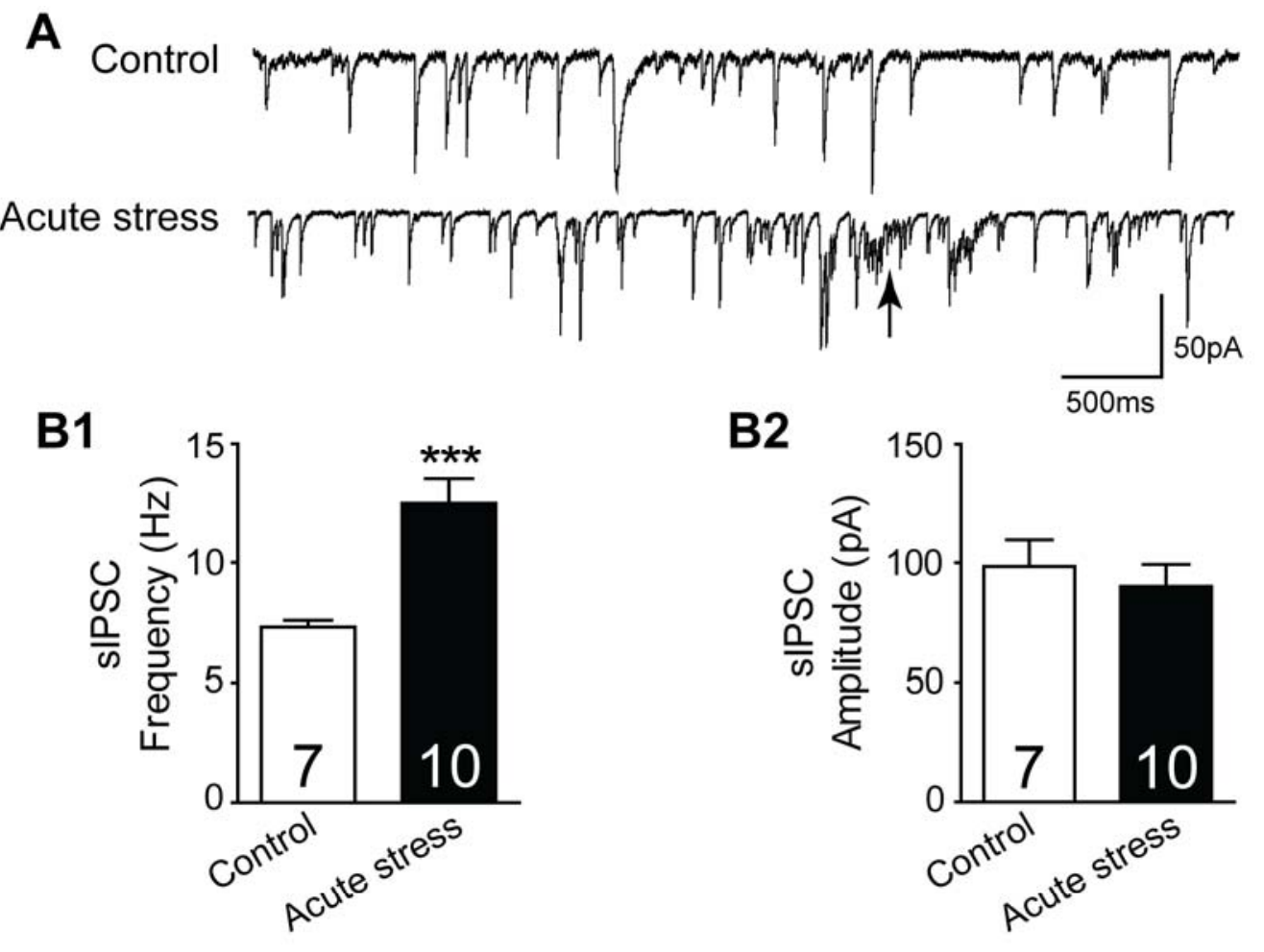

Figure 5. Acute stress increases hippocampal GABAergic transmission. A:

Representative voltage-clamp recordings of sIPSCs in CA1 pyramidal cells of control and acutely stressed rats. Acute stress significantly increased sIPSC frequency (B1), whereas the amplitude (B2) was not affected. Data are mean \pm SEM; numbers within the bars indicate the number of neurons recorded; ${ }^{* *} \mathrm{P}<0.001$. Six adult male rats were used for each group. Arrow: burst-like activities. Modified from Hu et al., 2010. 


\subsubsection{Effects of chronic stress on hippocampal GABAergic transmission}

\subsubsection{Physiological parameters after chronic restraint stress}

We then asked whether the elevation in hippocampal sIPSCs sustains following long-term stress during which the system is exposed to prolonged high level of stress hormones including corticosterone. To examine this, rats exposed to three weeks of restraint stress were used. To assess the physiological effects of chronic restraint stress, body weights were recorded daily throughout the experiment and adrenal weights were determined at the end of the experiment. Stress significantly reduced body weight gain, and two-way ANOVA (stress $\times$ time) revealed a significant main effect of stress $\left(F_{1,27}=606.6, P<0.001\right.$; Fig $\left.6 \mathrm{~A}\right)$. Furthermore, the chronic stress increased relative adrenal weights (unpaired $t$-test $t_{10}=2.579$, $P<0.05$; Fig $6 \mathrm{~B}$ ), which is in line with earlier findings that increased adrenal weights are reliable indicators of sustained HPA (hypothalamus-pituitary-adrenal)-axis hyperactivity (Magariños and McEwen, 1995; McLaughlin et al., 2007).
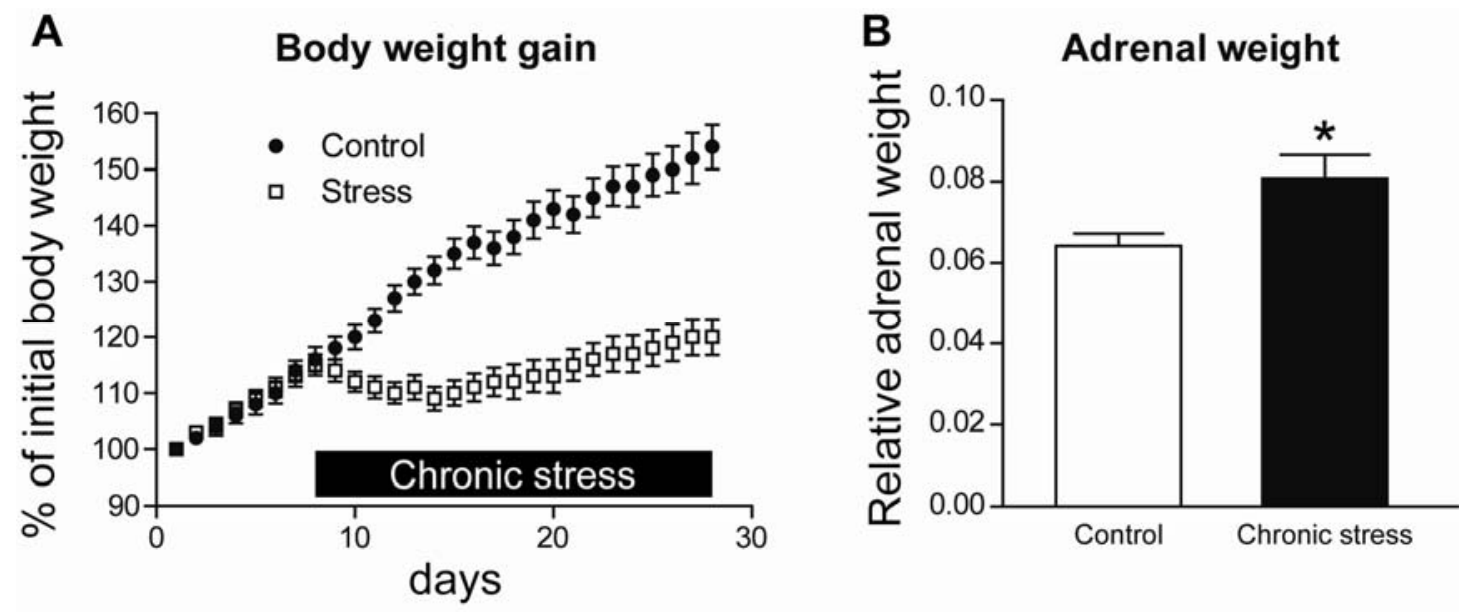

Figure 6. Physiological parameters in response to chronic stress. A: Body weight gain (Control: $\mathrm{n}=23$; Stress: $\mathrm{n}=22$ ), B: Relative weight of the adrenal glands ( $\mathrm{mg} / 100 \mathrm{~g}$ body weight; 6 animals per group). Data are mean \pm SEM. ${ }^{*} \mathrm{P}<0.05$. Modified from Hu et al., 2010. 


\subsubsection{2 $\mathrm{Ca}^{2+}$-dependent elevation of GABAergic transmission following chronic stress}

We next investigated whether long-term stress affected the occurrence of sIPSCs in CA1 pyramidal neurons. Exposure to chronic restraint stress resulted in a significant increase in the frequency of hippocampal sIPSCs (control: $5.9 \pm 0.3 \mathrm{~Hz}$; stress: $10.4 \pm 0.5 \mathrm{~Hz}$; unpaired $t$-test $t_{48}=9.279 ; P<0.0001 ;$ Fig. $\left.7 A, B\right)$, without significant changes in the sIPSC amplitude

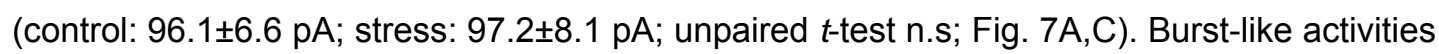
were also observed in 10 out of 16 cells recorded from this group.

Furthermore, the stress-induced enhancement of the sIPSC frequency could be normalized by the membrane permeable $\mathrm{Ca}^{2+}$ chelator EGTA-AM which reduces the concentration of intracellular free $\mathrm{Ca}^{2+}$. One-way ANOVA revealed a significant difference in the SIPSC frequency between the groups $\left(F_{(3,60)}=35.19 ; P<0.0001\right)$. Tukey's post hoc test showed a significant difference between the control and the chronic stress groups $(q=13.05 ; P<0.001)$. Bath application of EGTA-AM to the hippocampal slices of chronically-stressed animals normalized the sIPSC frequency to control level $(100 \mu \mathrm{M}$; stress+EGTA-AM: $6.4 \pm 0.4 \mathrm{~Hz}$; $\mathrm{q}=7.884, P<0.001$ vs stress; $\mathrm{q}=1.208$, n.s vs control; Fig. 7A,B,C). While in the control slices, EGTA-AM caused no significant changes in sIPSC (Fig. 7A,B,C). This shows that chronic stress induces an enhancement of GABAergic transmission, probably concomitant with an increased intracellular free $\mathrm{Ca}^{2+}$ in hippocampal interneurons.

Importantly, bath application of DEX $(25 \mathrm{nM})$ to the slices from the chronically stressed rats failed to elicit any significant change in sIPSCs of the CA1 pyramidal neurons (frequency: 10.2 $\pm 0.9 \mathrm{~Hz}$ before and $10.3 \pm 1.0 \mathrm{~Hz}$ after DEX, paired t-test, n.s.; amplitude: $87.2 \pm 6.4 \mathrm{pA}$ before and $85.3 \pm 5.1 \mathrm{pA}$ after DEX, paired t-test, n.s., Fig. 8). The origin of the loss of the DEX effect after chronic stress is yet to be determined (see Discussion). 
A Control

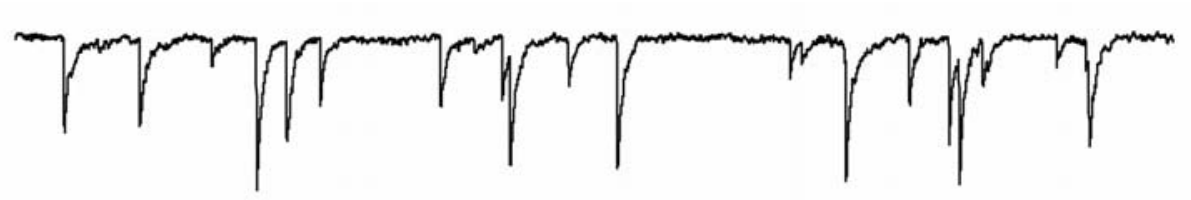

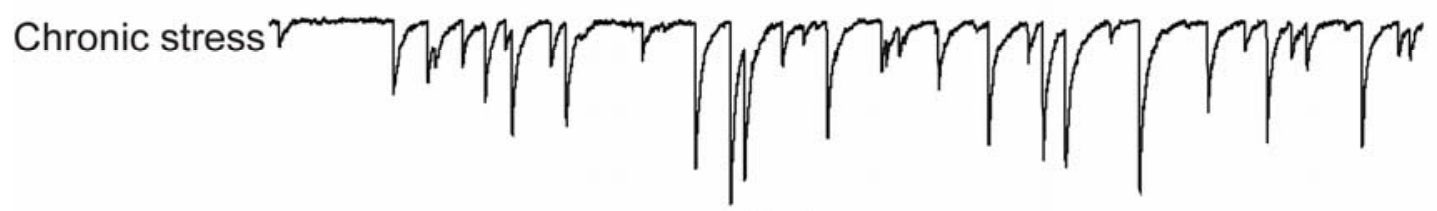

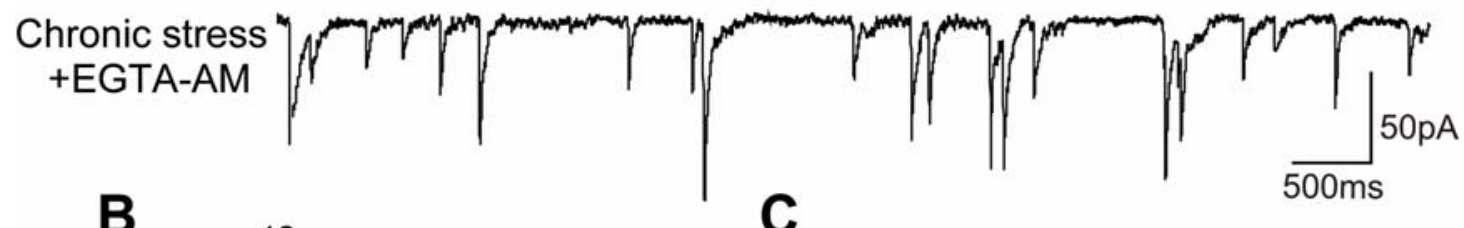

B

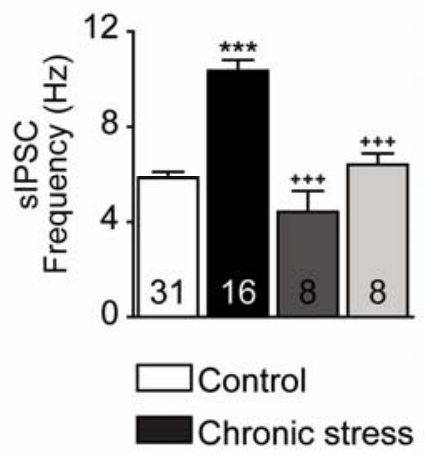

C

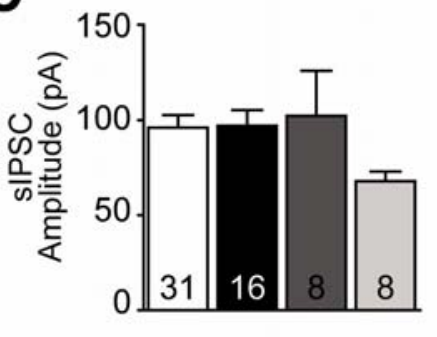

$\square$ Control+EGTA-AM

$\square$ Chronic stress+EGTA-AM

Figure 7. Chronic stress increases hippocampal GABAergic transmission. A:

Representative voltage-clamp recordings of sIPSCs in CA1 pyramidal cells of a control and a chronically stressed rat. Stress significantly increased sIPSC frequency $(B)$, whereas the amplitude was not affected $(C)$ (control: $n=14$; stress: $n=12$ ). The sIPSC frequency which was elevated by stress was normalized by EGTA-AM ( 7 animals per group). Data are mean $\pm S E M$; numbers within the bars indicate the number of neurons recorded; ${ }^{\star \star \star} \mathrm{P}<0.001$, vs. control; $+++\mathrm{P}<0.001$, vs. stress. Modified from Hu et al., 2010. 
A Chronic stress

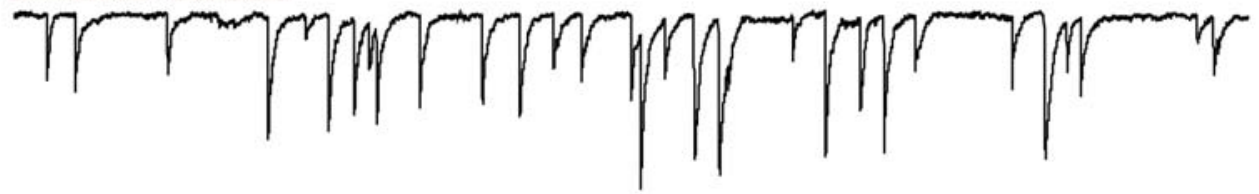

Chronic stress+DEX

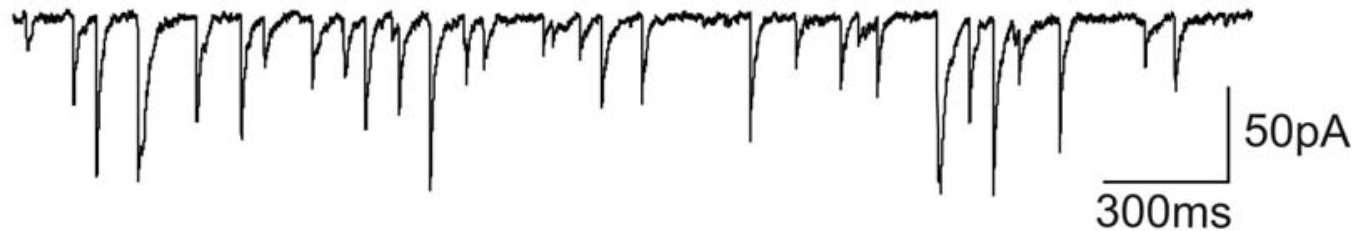

B1

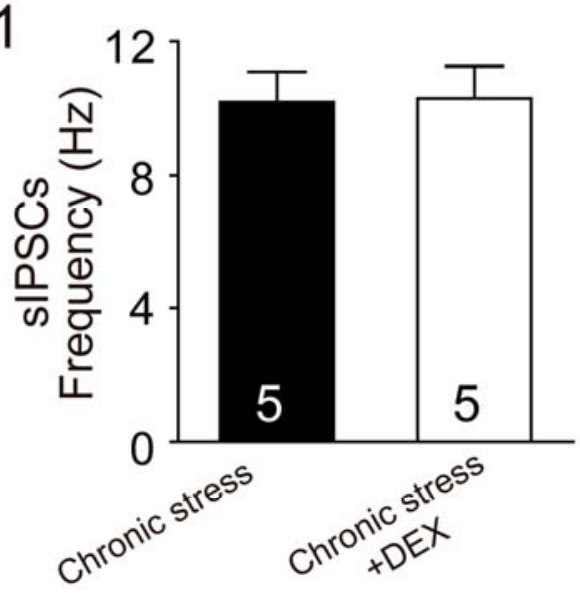

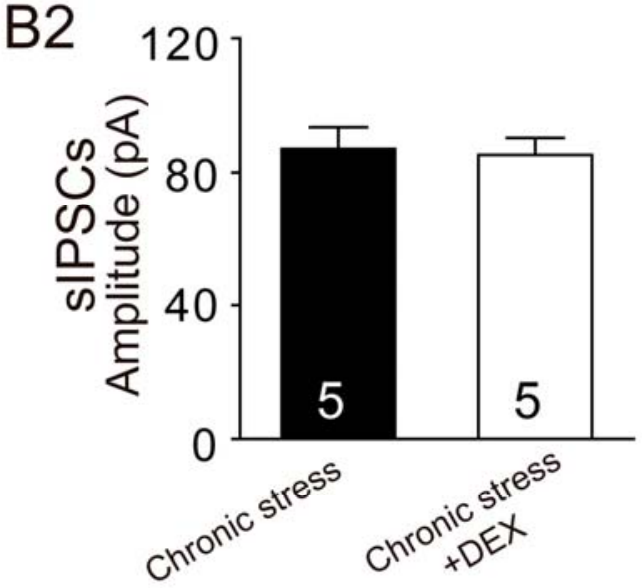

Figure 8. Bath application of DEX $(25 \mathrm{nM})$ failed to elicit any significant change in sIPSCs in the hippocampus of chronically stressed rats. A: Representative recordings of sIPSCs in a CA1 pyramidal cell from a stressed rat before and after the application of the GR agonist DEX. B: Numerical data of the means of frequency (B1) and amplitude (B2) of sIPSCs before (black bar) and after DEX (gray bar) ( 5 neurons recorded from 5 stressed animals). Data are mean \pm SEM. Modified from Hu et al., 2010. 


\subsubsection{Effects of chronic stress on the PV interneuron network in the hippocampus}

\subsubsection{Effects of chronic stress on numbers of PV-and CCK-immunoreactive neurons}

Considering i) the theory of functional dichotomy of $\mathrm{PV}+$ and $\mathrm{CCK}+$ neurons proposed by Freund (Freund and Katona, 2003) and ii) our present results which indicate that DEX facilitates the release of endogenous CCK (Fig. 4) which specifically stimulates PV-expressing neurons, we asked whether these two subtypes of perisomatic interneurons are differentially affected by chronic stress. We examined effects of chronic restraint stress on the structural integrity of PV and CCK interneurons. The number of these neurons in the dorsal hippocampus of control and chronically stressed rats were quantified. Chronic stress decreased the number of PV-immunoreactive neurons in all hippocampal subregions: $31 \%$ reduction in dentate gyrus (unpaired t-test, $P<0.01$, Fig. 9 C), $23 \%$ reduction in CA2-3 (unpaired t-test, $P<0.01$, Fig. $9 \mathrm{C}$ ), and $36 \%$ reduction in CA1 (unpaired t-test, $P<0.01$, Fig. 9A1,A2,C). In contrast, the number of CCK-immunoreactive neurons remained unaltered (Fig. 9B1,B2,D).

\subsubsection{Chronic stress effects on CCK-induced rhythmic burst activity originating from the PV interneurons}

We then ask whether the above differential effects of chronic stress on the number of PV and CCK-immunoreactive neurons affect the function of PV and CCK interneurons. It is reported that an elaborately regulated $\mathrm{Ca}^{2+}$ signaling is essential for the temporal precision of the output from interneurons (Hefft and Jonas, 2005). Since our data indicated that chronic stress induced an excessive intracellular free $\mathrm{Ca}^{2+}$ in the hippocampal interneurons (Fig. 7), we decided to test whether chronic stress affects the ability of the perisomatic interneurons to generate rhythmic firing which is of great importance in controlling the timing of pyramidal neuron action potentials. It is known that in the hippocampus, both CCK analogs and the muscarinic acetylcholine receptor agonist carbachol can provoke rhythmic SIPSCs in vitro. Carbachol-induced rhythmic IPSCs are inhibited by endocannabinoids, by $\mathrm{N}$-type calcium channel blockers and by activation of $\mathrm{GABA}_{B}$ receptors (Karson et al., 2008). In contrast, 
CCK-triggered IPSCs are inhibited by P/Q-type calcium channel blockers, but are insensitive to endocannabinoids (Karson et al., 2008). Given the distinct cellular properties of CCK and PV interneurons, carbachol presumably triggers rhythmic sIPSCs originating from CCK interneurons whereas CCK stimulates sIPSCs from PV interneurons (Karson et al., 2008). In the present study, bath application of carbachol $(5 \mu \mathrm{M})$ induced a rhythmic pattern of sIPSCs in hippocampal slices of control rats (Fig. 10A1,A2). Autocorrelation analysis of 10-sec stretches of sIPSCs (selected from shortly after the onset of the enhancement) in all 8 cells showed regular-repeating peaks. After carbachol application, power spectral analysis of the same stretches from each cell revealed a significant enhancement in the total power and more importantly, a sharp peak power within the theta frequency range, $4-14 \mathrm{~Hz}$ (relative theta power was $46.7 \pm 1.1 \%$ before carbachol and $57.7 \pm 1.1 \%$ after carbachol; paired t-test $t_{7}=8.326, P<0.0001$; Fig. 10A2,C). Similarly, bath application of the CCK analog CCK8-S $(1 \mu \mathrm{M})$ induced rhythmic sIPSCs which were manifested by regular-repeating peaks in the autocorrelation analysis, and a significant increase of power specifically in the theta frequency range in all 8 tested cells (relative theta power was $47.1 \pm 1.0 \%$ before and $60.2 \pm 1.9 \%$ after CCK application; paired t-test $t_{7}=6.374, P<0.0001$; Fig. $\left.11 \mathrm{~A} 1, \mathrm{~A} 2, \mathrm{C}\right)$. Consistent with previous studies, the rhythmicity triggered by the CCK analog was less variable and more persistent compared to that induced by carbachol.

In hippocampal slices from the chronically stressed rats the response to carbachol was similar to that in controls (relative theta power before carbachol: $46.2 \pm 1.5 \%$, after carbachol: $59.4 \pm 1.9 \%$; paired t-test $t_{7}=12.28, P<0.0001$; Fig. 10B1,B2,C). Two-way ANOVA of repeated measures of data from the control and the chronic stress group also revealed no significant effect of stress ( $F=0.1567 ; n . s)$. However, CCK8-S failed to induce rhythmicity in all 10 tested cells. In contrast to control, the CCK agonist induced an arrhythmic pattern in the slices from the stressed rats as indicated by flat autocorrelation curves. Similarily, CCK8-S did not change relative theta power in stressed rats (before CCK $49.4 \pm 1.2 \%$; after CCK $48.7 \pm 1.6 \%$; paired t-test $t_{9}=0.730$, n.s; Fig. 11B1,B2,C) despite a massive increase in the total power. This demonstrates that in addition to the enhancement of GABAergic transmission, the temporal precision in firing of the PV interneurons is impaired after chronic stress, whereas the same parameter remains unaffected in CCK interneurons. 

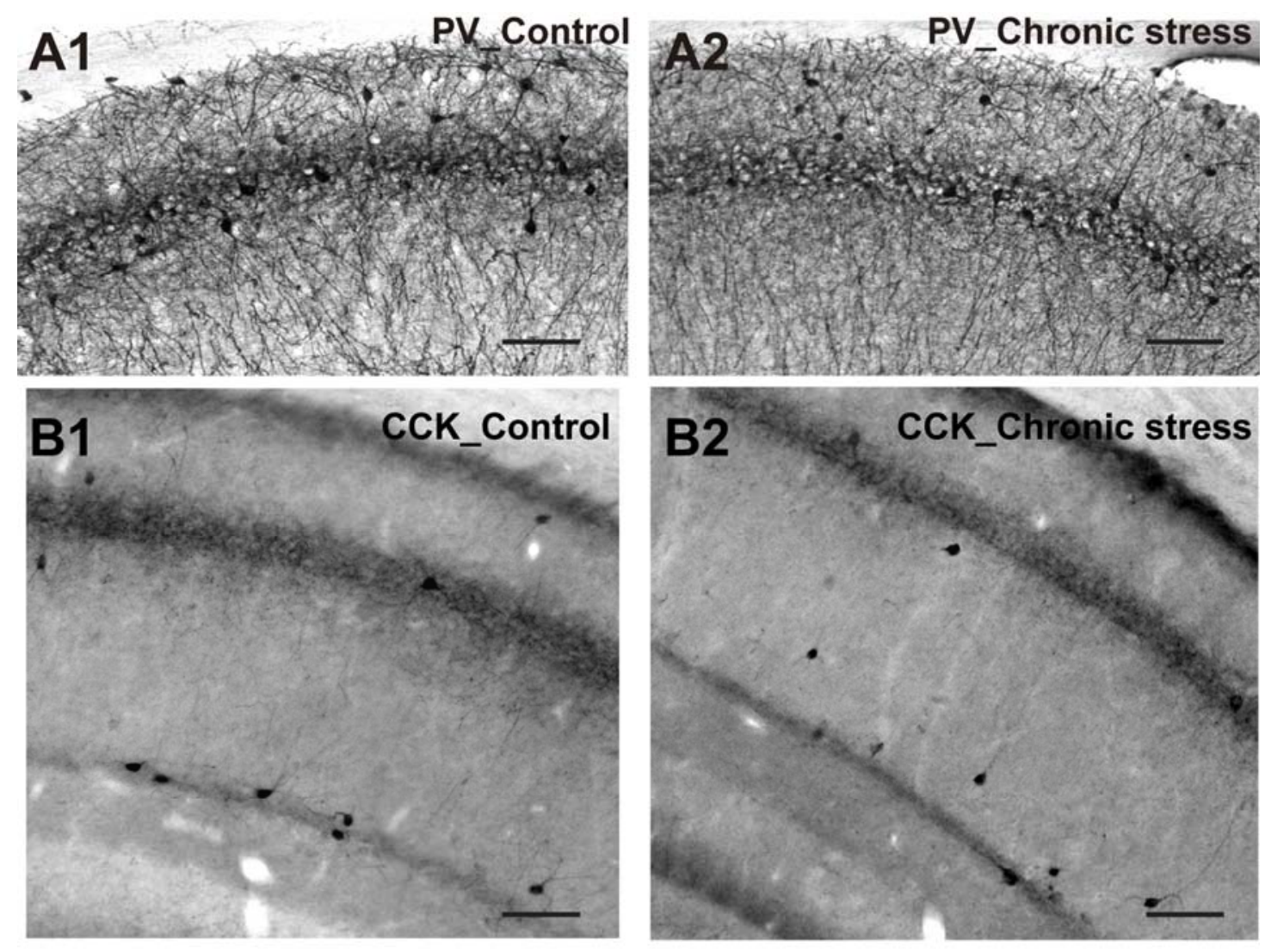

C Number of PV-positive cells
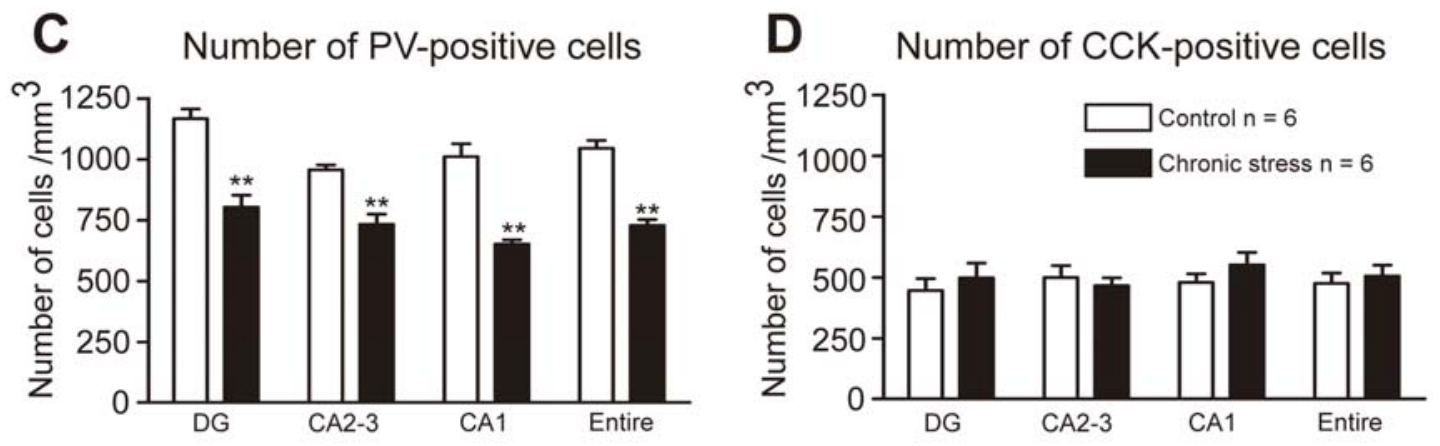

Figure 9. Effects of chronic stress on the number of PV- and CCK-immunoreactive cells in the hippocampus. A: Representative examples of parvalbumin stained CA1 sections from a control (A1) and a stressed rat (A2). B: Representative examples of cholecystokinin stained CA1 sections from a control (B1) and a stressed (B2) rat. Stress significantly decreased the number of $P V$ interneurons in all hippocampal subareas (C), whereas the number of CCK interneurons was not affected (D). This indicates that chronic stress affects the structural integrity of PV but not of CCK interneurons. Data are mean \pm SEM; 6 animals per group were analyzed; ${ }^{\star *} P<0.01$. DG: dentate gyrus. Modified from Hu et al., 2010. 


\section{A1}

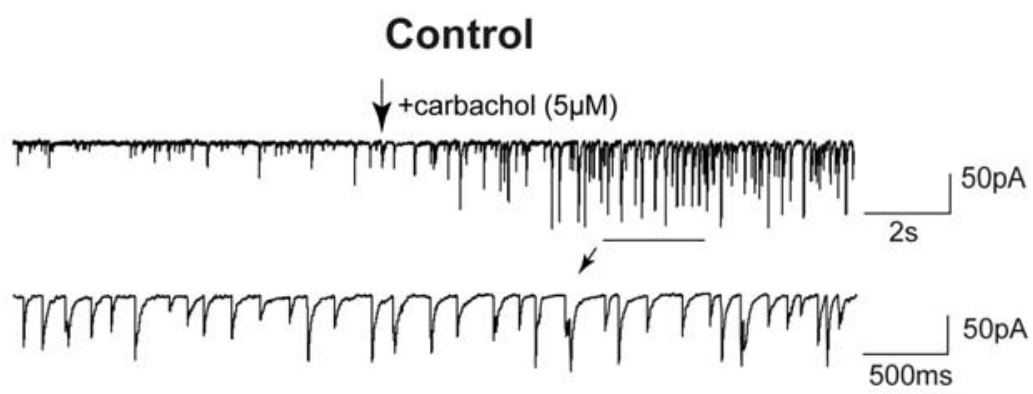

A2
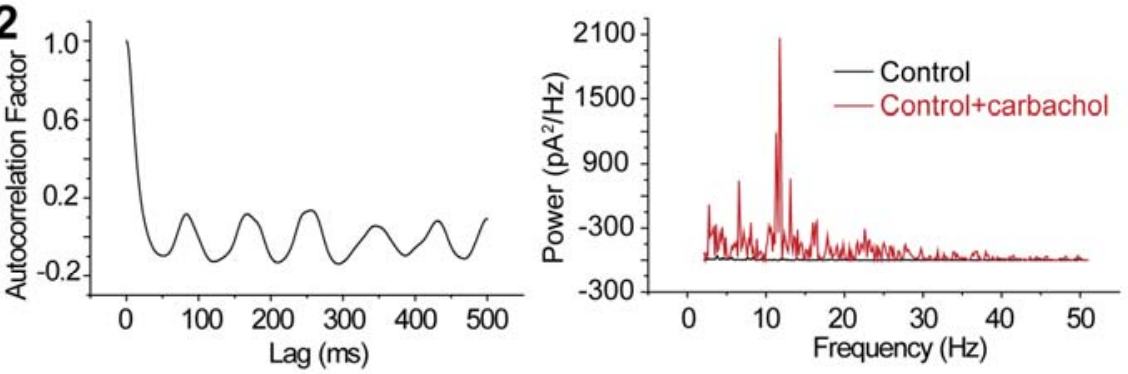

B1

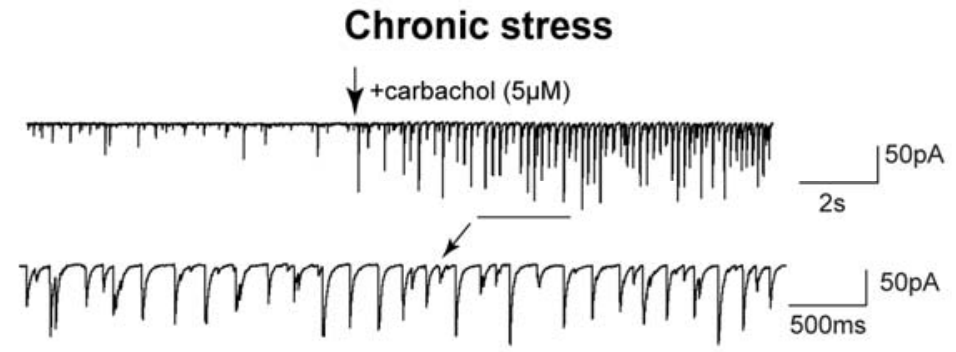

\section{B2}
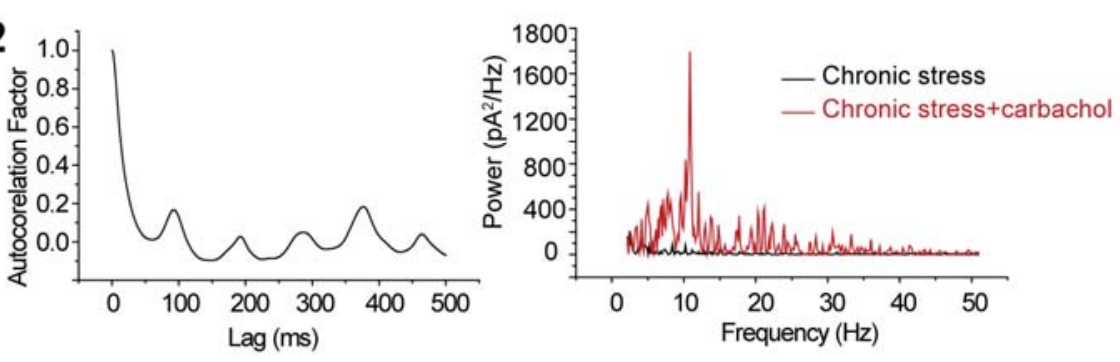

C

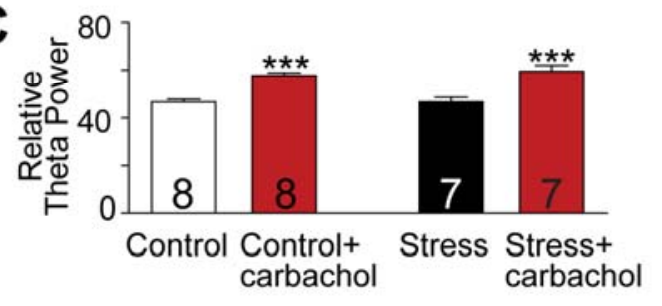

Figure 10. The ability of CCK interneurons to generate rhythmic sIPSCs in response to carbachol was unaltered after chronic stress. A: Representative data from a control animal. Bath application of carbachol induced rhythmic sIPSCs. A1: Representative recordings of sIPSCs in a CA1 pyramidal cell before and during washing in of carbachol. Lower traces are time expanded from the indicated segments in the upper traces. A2: Autocorrelation (left) and spectral power analysis (right) of 10-sec stretches from the representative recordings after the experimental treatment. Graphs are representative of autocorrelation and spectra from all recorded cells. B: Representative data from a stressed animal. In slices from chronically stressed rats carbachol had the same effect as in controls. This indicates that the temporal precision in firing of the CCK interneuron remains unaffected by chronic stress. C: Numerical data of relative theta power before and after the treatments. Data are mean \pm SEM. Numbers within the bars indicate numbers of neurons tested for each animal group (7 animals per group) and experimental condition; same cells in all conditions; ${ }^{* \star *} \mathrm{P}<0.001$. Modified from Hu et al., 2010. 
A1

\section{Control}

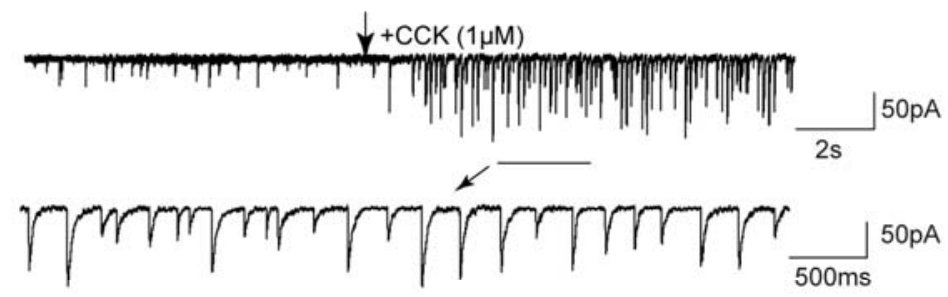

A2
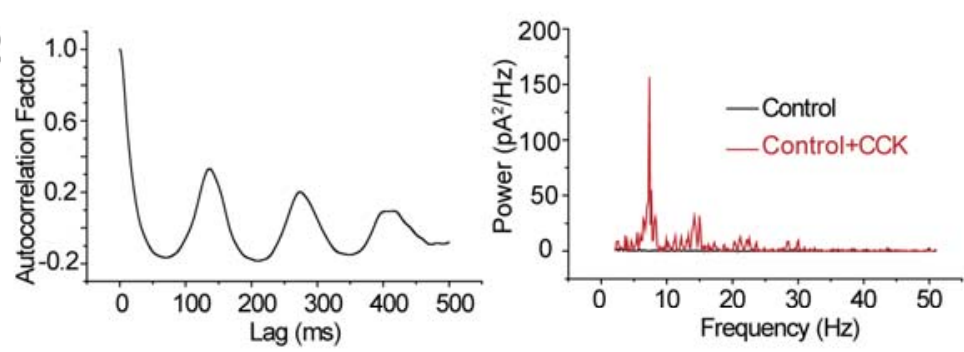

B1

Chronic stress

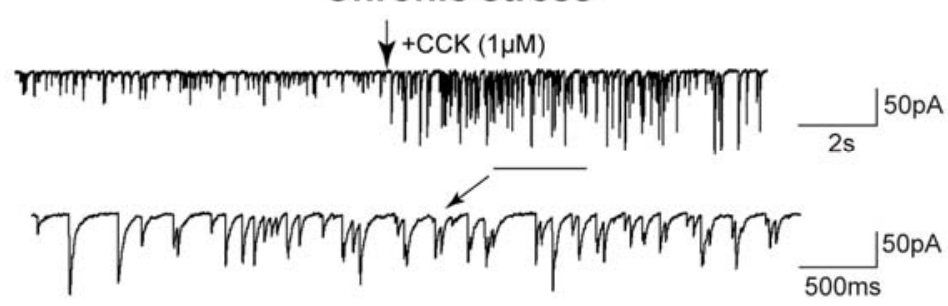

B2
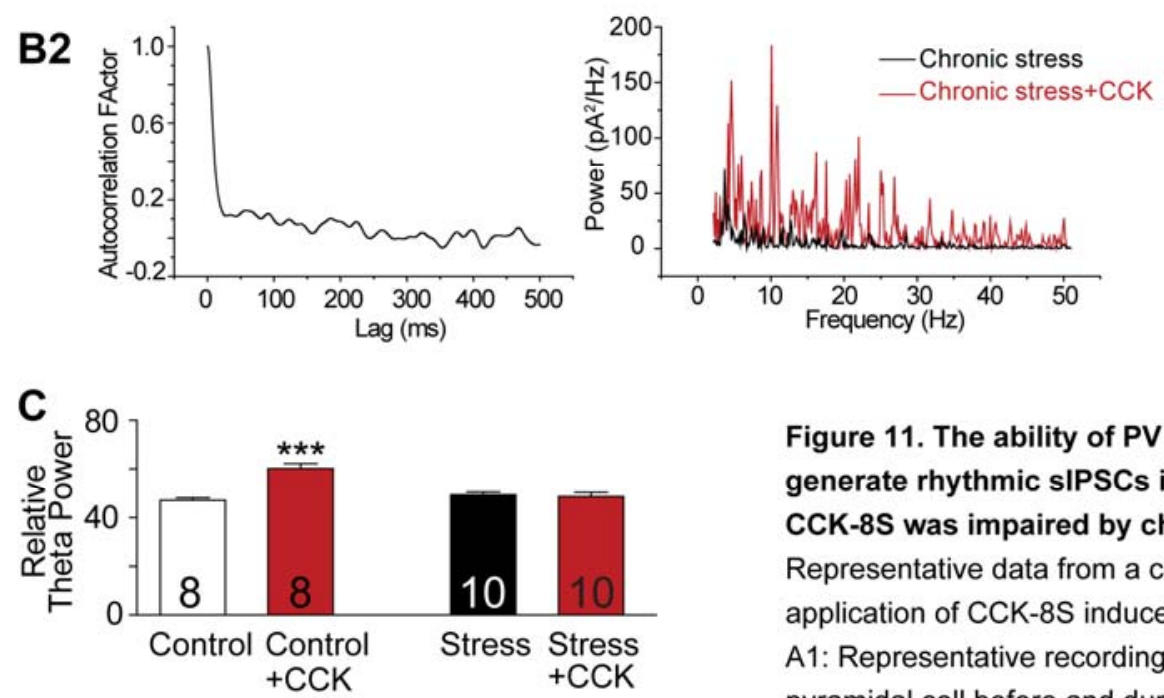

Figure 11. The ability of PV interneurons to generate rhythmic SIPSCs in response to CCK-8S was impaired by chronic stress. A: Representative data from a control animal. Bath application of CCK-8S induced rhythmic SIPSCs. A1: Representative recordings of sIPSCs in a CA1 pyramidal cell before and during washing in of

CCK-8S. Lower traces are time expanded from the indicated segments in the upper traces. A2:

Autocorrelation (left) and spectral power analysis (right) of 10-sec stretches from the representative recordings after the experimental treatment. Graphs are representative of autocorrelation and spectra from all recorded cells. B: Representative data from a stressed animal. In contrast to control, CCK-8S failed to induce a rhythmic SIPSC pattern in chronically-stressed animals. This indicates that the temporal precision in firing of the PV interneurons is impaired after chronic stress. C: Numerical data of relative theta power before and after the treatments. Data are mean \pm SEM. Numbers within the bars indicate numbers of neurons tested for each animal group (7 animals per group) and experimental condition; same cells in all conditions; ${ }^{* \star *} \mathrm{P}<0.001$. Modified from Hu et al., 2010.Modified from Hu et al., 2010. 


\subsubsection{Effects of chronic stress on endocannabinoid mediated modulation of CCK interneuron activity in the hippocampus}

Previous evidence suggested that chronic stress regulates endocannabioid signaling in the brain ((Hill et al., 2005; 2009; Patel et al., 2005; 2009; Rademacher et al., 2008; Rossi et al., 2008). Immunostaining in the hippocampus revealed that CB1 receptors are almost exclusively localized on the axon terminals of CCK interneurons (Hájos et al., 2000). Although chronic restraint stress has no impact on the ability of the CCK interneuron network to generate rhythmic IPSCs in response to carbachol, it is important to ask whether chronic stress may affect hippocampal CCK interneuron network activity with regard to its sensitivity to endocannabinoid mediated modulation.

In consistence with a previous report (Reich et al., 2005), a single 5 s-depolarizing voltage step significantly reduced carbachol-induced IPSC frequency which sustained even 2 min after depolarization (Fig 12A1,C1, Table 1). The DSI of carbachol-induced IPSCs was completely abolished by a 15 min incubation with the CB1 inverse agonist AM251 (10 $\mu \mathrm{M})$

(Fig 12A2,C2, Table 1; repeated two-way ANOVA drug $\times$ time $F_{(1,66)}=19.24, P=0.0011$ ), confirming that this effect was mediated by endocannabinoid signaling through CB1 receptors. By contrast, carbachol-induced IPSCs recorded in the hippocampal slices from chronically stressed animals were substantially resistant to depolarization induced suppression (Fig 12B1,C1; Table 1). Repeated two-way ANOVA (stress $\times$ time) revealed a significant main effect of stress (Fig 12C1, $\left.F_{1.66}=15.08, P=0.0025\right)$. In addition, AM251 incubation had no significant effect on carbachol-IPSCs following depolarization (Fig 12B2,C3, repeated two-way ANOVA drug $\times$ time $F_{1,72}=0.17, P=0.6906$; Table 1). These data demonstrate that chronic stress impairs the endocannabinoid mediated modulation of GABAergic transmission in the hippocampus which is consistence with previous reports showing that chronic stress downregulates hippocampal CB1 receptor expression (Hill et al., 2005). Because CB1 receptors in the hippocampus are expressed almost exclusively on CCK expressing interneurons, the impairment of DSI suggests an alteration in the functioning of the CCK interneurons within the hippocampal network, although the temporal precision in firing of these cells is still intact after chronic stress. 
Control

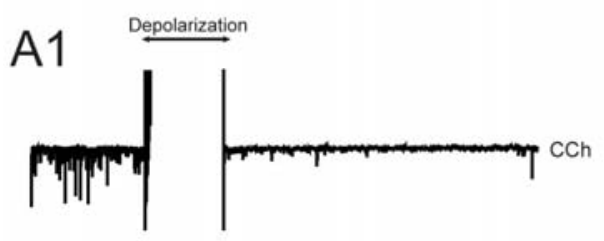

A2

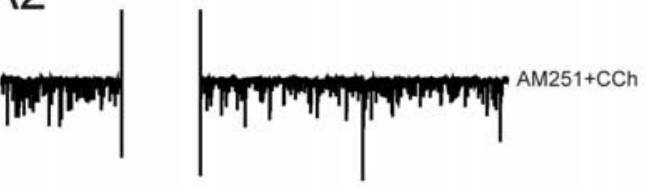

C1

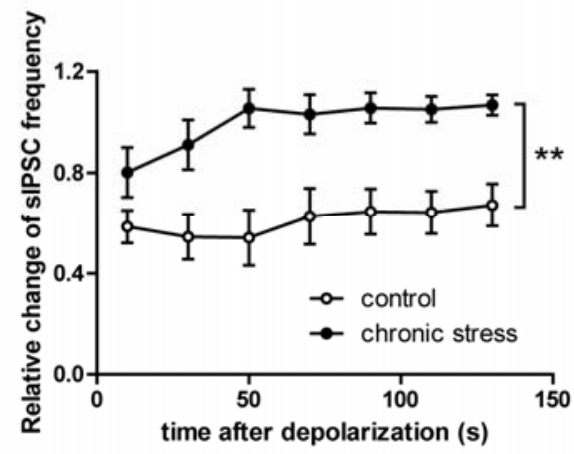

C3

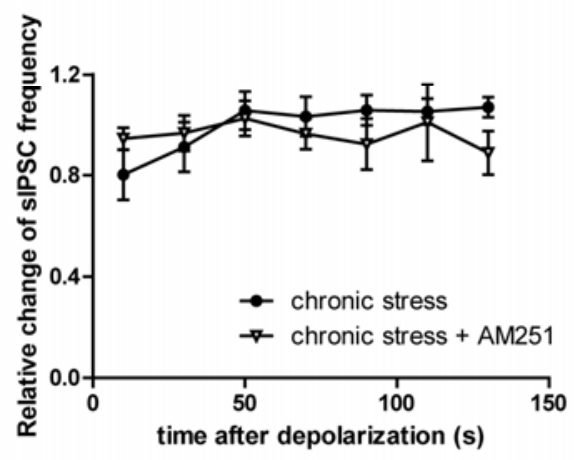

Stress

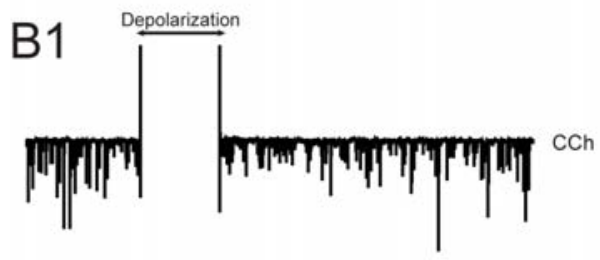

B2

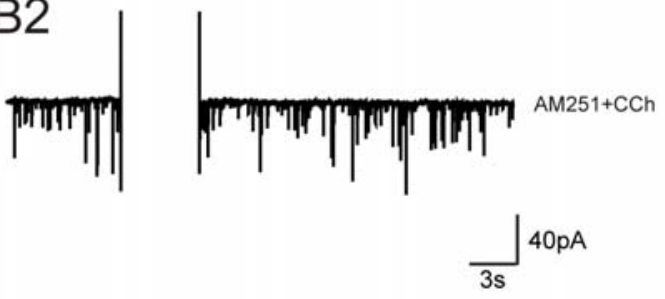

C2

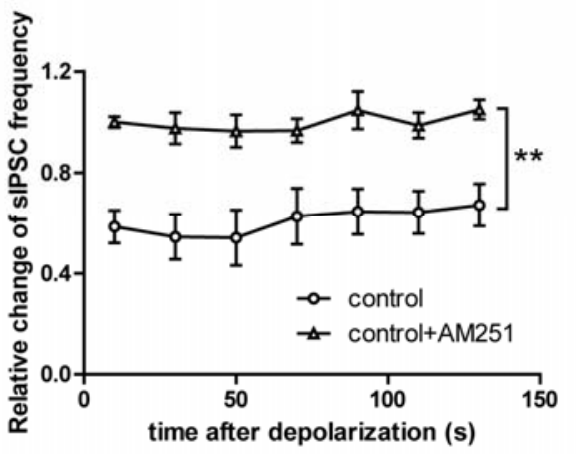

Figure 12. Chronic stress impairs endocannabinoid mediated suppression of CCK interneuron activity. A, B: Representative recordings of $D S I$ in a CA1 pyramidal cell from a control $(A)$ and a chronically stressed (B) animal. C: Time course of DSI (graphs show relative changes). $\mathrm{C} 1$ : In the control group, a single $5 \mathrm{~s}$-depolarizing

voltage step significantly reduced the frequency of carbachol-induced IPSC whereas in the chronic stress group, carbachol-induced IPSCs were resistant to depolarization. C2: DSI in the control group was abolished in the presence of the CB1 antagonist AM251. C3: AM251 incubation had no significant effect in the slices from chronically stressed animals. Repeated two-way ANOVA, ${ }^{* *} \mathrm{P}<0.01$. 
Table 1: Numerical data of relative changes of carbachol-induced IPSC frequency following depolarization in CA1. Mean is calculated as the ratio of IPSC frequency after depolarization to that before depolarization. Statistics: one-way ANOVA, followed by Dunnett's multiple comparison test, with mean normalized frequency before depolarization being 1.0. SD: standard deviation.

Control

\begin{tabular}{|l|l|l|c|c|}
\hline \multirow{2}{*}{ Time after depolarization } & \multirow{2}{*}{ Mean } & \multirow{2}{*}{ SD } & \multicolumn{2}{|c|}{ Dunnett's Multiple Comparison Test } \\
\cline { 4 - 5 } & & & $\mathrm{q}$ & $P$ \\
\hline 10s & 0.586 & 0.160 & 6.477 & $<0.001$ \\
\hline 30s & 0.545 & 0.219 & 7.120 & $<0.001$ \\
\hline 50s & 0.541 & 0.269 & 7.177 & $<0.001$ \\
\hline 70s & 0.626 & 0.272 & 5.843 & $<0.001$ \\
\hline 90s & 0.646 & 0.220 & 5.538 & $<0.001$ \\
\hline 110s & 0.643 & 0.206 & 5.587 & $<0.001$ \\
\hline 130s & 0.672 & 0.204 & 5.134 & $<0.001$ \\
\hline
\end{tabular}

Chronic stress

\begin{tabular}{|l|c|c|c|c|}
\hline \multirow{2}{*}{ Time after depolarization } & \multirow{2}{*}{ Mean } & \multirow{2}{*}{ SD } & \multicolumn{2}{|c|}{ Dunnett's Multiple Comparison Test } \\
\cline { 3 - 5 } & & & $\mathrm{q}$ & $P$ \\
\hline $\mathbf{1 0 s}$ & 0.801 & 0.260 & 2.707 & $\mathrm{~ns}$ \\
\hline $\mathbf{3 0 s}$ & 0.911 & 0.260 & 1.219 & $\mathrm{~ns}$ \\
\hline $\mathbf{5 0 s}$ & 1.054 & 0.198 & 0.741 & $\mathrm{~ns}$ \\
\hline $\mathbf{7 0 s}$ & 1.031 & 0.205 & 0.420 & $\mathrm{~ns}$ \\
\hline $\mathbf{9 0 s}$ & 1.056 & 0.158 & 0.760 & $\mathrm{~ns}$ \\
\hline $\mathbf{1 1 0 s}$ & 1.050 & 0.136 & 0.687 & $\mathrm{~ns}$ \\
\hline $\mathbf{1 3 0}$ & 1.068 & 0.106 & 0.924 & $\mathrm{~ns}$ \\
\hline
\end{tabular}


Control + AM251

\begin{tabular}{|l|c|l|c|c|}
\hline \multirow{2}{*}{ Time after depolarization } & \multirow{2}{*}{ Mean } & \multirow{2}{*}{ SD } & \multicolumn{2}{|c|}{ Dunnett's Multiple Comparison Test } \\
\cline { 3 - 5 } & & & $\mathrm{q}$ & $P$ \\
\hline $\mathbf{1 0 s}$ & 1.001 & 0.057 & 0.005 & $\mathrm{~ns}$ \\
\hline $\mathbf{3 0 s}$ & 0.975 & 0.165 & 0.440 & $\mathrm{~ns}$ \\
\hline $\mathbf{5 0 s}$ & 0.964 & 0.169 & 0.641 & $\mathrm{~ns}$ \\
\hline $\mathbf{7 0 s}$ & 0.966 & 0.125 & 0.602 & $\mathrm{~ns}$ \\
\hline $\mathbf{9 0 s}$ & 1.047 & 0.197 & 0.833 & $\mathrm{~ns}$ \\
\hline $\mathbf{1 1 0 s}$ & 0.987 & 0.133 & 0.235 & $\mathrm{~ns}$ \\
\hline $\mathbf{1 3 0}$ & 1.050 & 0.103 & 0.893 & $\mathrm{~ns}$ \\
\hline
\end{tabular}

Chronic stress + AM251

\begin{tabular}{|l|c|c|c|c|}
\hline \multirow{2}{*}{ Time after depolarization } & \multirow{2}{*}{ Mean } & \multirow{2}{*}{ SD } & \multicolumn{2}{|c|}{ Dunnett's Multiple Comparison Test } \\
\cline { 4 - 5 } & & & $\mathrm{q}$ & $P$ \\
\hline 10s & 0.944 & 0.114 & 0.660 & $\mathrm{~ns}$ \\
\hline 30s & 0.966 & 0.185 & 0.403 & $\mathrm{~ns}$ \\
\hline $\mathbf{5 0 s}$ & 1.024 & 0.183 & 0.279 & $\mathrm{~ns}$ \\
\hline $\mathbf{7 0 s}$ & 0.962 & 0.162 & 0.442 & $\mathrm{~ns}$ \\
\hline $\mathbf{9 0 s}$ & 0.922 & 0.265 & 0.918 & $\mathrm{~ns}$ \\
\hline $\mathbf{1 1 0 s}$ & 1.008 & 0.401 & 0.095 & $\mathrm{~ns}$ \\
\hline $\mathbf{1 3 0}$ & 0.887 & 0.228 & 1.328 & $\mathrm{~ns}$ \\
\hline
\end{tabular}


3.2 Part II: Stress effects in the mPFC

3.2.1 A lateralized chronic stress effect on the number of PV-immunoreactive cells in the MPFC

To elucidate stress effects on the prefrontal cortex pilot experiments on the mPFC were performed. The first experiment was designed to examine whether chronic stress has any effect on the number of PV-immunoreactive interneurons. Exposure to 3 weeks of restraint stress resulted in a significant reduction in the total number of PV-immunoreactive cells in the right $\operatorname{mPFC}\left(-16 \%\right.$; unpaired $t$-test, $\mathrm{t}_{14}=2.775, P<0.05$; Table 2; Fig. $\left.13 \mathrm{~A}\right)$. By contrast, the total number of PV interneurons in the left mPFC was unaffected $\left(-1 \%\right.$; unpaired $t$-test, $t_{14}$ $=0.1947 ;$ n.s; Table 2; Fig.13B) .

Table 2: Stereological results: Mean estimated total numbers of PV-immunoreactive interneurons in right and left mPFC after chronic restraint stress

\begin{tabular}{ccc}
\hline & Control & Stress \\
\hline Right mPFC & & \\
Mean total number & 15556 & 13033 \\
SD & 1846 & 1790 \\
Mean CE & 0.04409 & 0.04800 \\
CV = SD/mean & 0.1187 & 0.1373 \\
$\mathrm{BCV}^{2}$ (in \% of $\mathrm{CV}^{2}$ ) & $86 \%$ & $88 \%$
\end{tabular}

Left MPFC

Mean total number $\quad 14237 \quad 14088$

$\begin{array}{ccc}\text { SD } & 1485 & 1572 \\ \text { Mean CE } & 0.04572 & 0.04598 \\ \text { CV = SD/mean } & 0.1043 & 0.1116\end{array}$




\section{$\mathrm{BCV}^{2}\left(\right.$ in $\%$ of $\left.\mathrm{CV}^{2}\right) \quad 81 \% \quad 83 \%$}

CE: coefficient of error, mean CE was calculated $\sqrt{\operatorname{mean}(C E)^{2}}$;

$\mathrm{CV}$ : coefficient of variation;

SD: standard deviation;

$\mathrm{BCV}$, biological variance representing precision of the estimation, where $\mathrm{CV}^{2}=\mathrm{CE}^{2}+\mathrm{BCV}^{2}$.

A

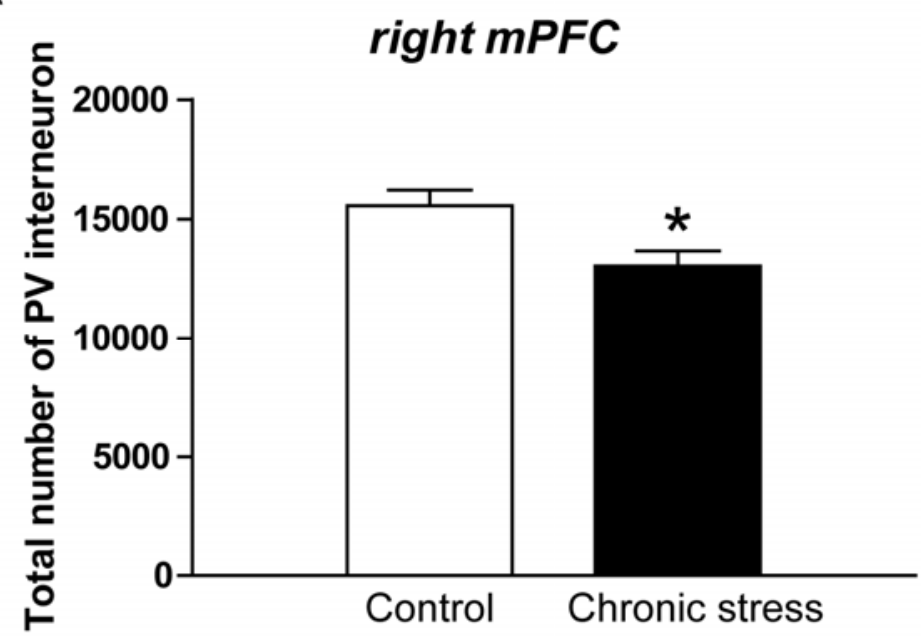

B

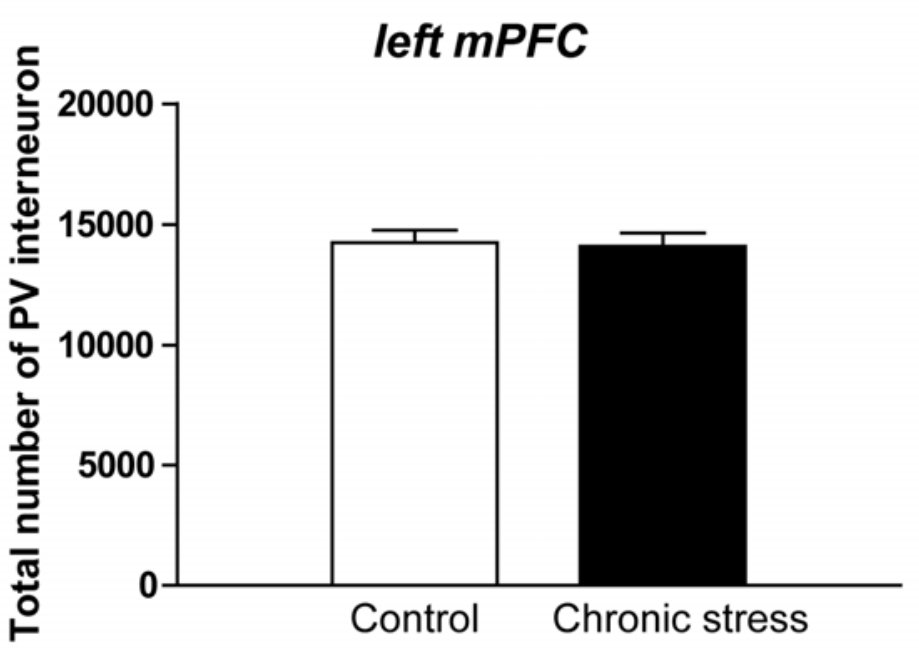

Figure 13. Lateralized effects of chronic stress on the number of

PV-immunoreactive cells in the mPFC. Chronic stress reduces the total number of PV-immunoreactive cells in the right (A) but not in the left mPFC (B). Data are mean $\pm S E M ; ~ 8$ animals per group were analyzed; ${ }^{*} P<0.05$. 


\subsubsection{Lateralized effects of acute stress on the GABAergic transmission in the mPFC}

The hemispheric asymmetry with respect to the change in PV cell number after chronic stress is not surprising. In fact, ample evidences showed similar lateralized regulations in stress responses in the mPFC before (Carlson et al., 1991; 1993; Gratton et al., 2005; Perez-Cruz et al., 2007; 2009). The next questions we then asked in our study were: 1) did lateralized changes in the mPFC GABAergic network already occur at an earlier stage of the stress period and 2) if yes, what were the potential factors that mediate these processes. To answer this, preliminary studies were conducted in animals that were acutely stressed.

Following 30 min of restraint stress, an increase in sIPSC frequency in the right mPFC but not in the left mPFC was observed. Two-way ANOVA (stress $\times$ hemisphere) revealed a main effect of acute stress on sIPSC frequency $\left(F_{1,44}=15.36, P<0.001\right.$; Fig. 14A,B1 $)$ with the significant increase occurring in layer II/III pyramidal cells of the right mPFC (control: $9.6 \pm 0.8$ $\mathrm{Hz}$; acute stress: $16.3 \pm 1.7 \mathrm{~Hz} ; \mathrm{t}=4.462 ; P<0.001$ Bonferroni post-hoc tests, Fig. 14A right,B1) but not of the left mPFC (control: $10.0 \pm 0.6 \mathrm{~Hz}$; acute stress: $11.5 \pm 1.1 \mathrm{~Hz}$; $\mathrm{t}=1.033$; n.s Bonferroni post-hoc tests, Fig. 14A left,B1). Similarly, acute stress significantly upregulated mIPSC frequency $\left(F_{1,34}=4.309, P<0.05\right.$ two-way ANOVA) recorded in the right (control: 3.9 $\pm 0.4 \mathrm{~Hz}$; acute stress: $6.8 \pm 0.9 \mathrm{~Hz} ; \mathrm{t}=3.195 ; P<0.01$ Bonferroni post-hoc tests) but not in the left hemisphere (control: $3.7 \pm 0.5 \mathrm{~Hz}$; acute stress: $3.3 \pm 0.4 \mathrm{~Hz} ; \mathrm{t}=0.5543 ; n . s$ ). By contrast, the amplitudes of both SIPSCs and mIPSCs were unaffected by acute stress in both hemispheres (sIPSC amplitude $F_{1,46}=0.5169$ n.s, Fig. 14B2; mIPSC amplitude $F_{1,34}=1.353$ n.s; two-way ANOVA stress $\times$ hemisphere). The result that mIPSCs were changed only in the frequency indicates that the observed stress effects originated from the presynaptic sites. Interestingly, we found that the acute stress induced increase in the GABAergic activity could be normalized by blocking the dopamine D1-like receptor. A 15 min incubation with the D1/5 receptor antagonist (SCH 23390) completely abolished the acute stress effect on the sIPSC frequency in right mPFC (control vs. acute-stress plus SCH 23390: $q=2.309, n . s$; acute stress vs. acute stress plus D1-antagonist: $q=6.276, P<0.001$; one-way ANOVA followed by Tukey's multiple comparison test; Fig. 14B1). Interestingly, incubating the brain slices from the acutely stressed animals with $\mathrm{SCH} 23390$ also resulted in a small but significant reduction 
in the sIPSC frequency recorded from the left mPFC pyramidal cell (acute stress vs. acute stress plus SCH 23390: q=3.927, $P<0.05$; one-way ANOVA followed by Tukey's multiple comparison test; Fig. 14B1); though this reduction did not produce any significant difference in sIPSC frequency compared to the control group (control vs. acute stress plus SCH 23390 : $\mathrm{q}=2.555$, n.s; one-way ANOVA followed by Tukey's multiple comparison test; Fig. 14B1).

These data suggested that acute stress results in increased GABAergic transmission specifically in layer II/III of the right but not in the left mPFC. An enhanced intrinsic dopaminergic signaling, probably with more pronounced changes in the right mPFC, might be associated with the observed stress effect. However, at this point we still can not rule out the possibility that SCH 23390 by itself has an effect on sIPSC frequency in the mPFC.

A left mPFC right $M P F C$

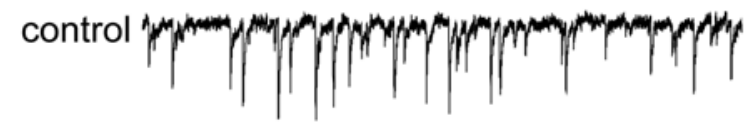

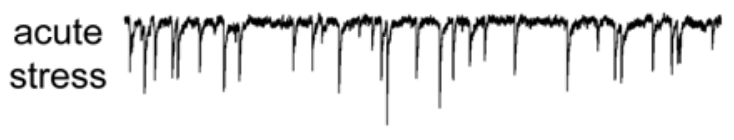

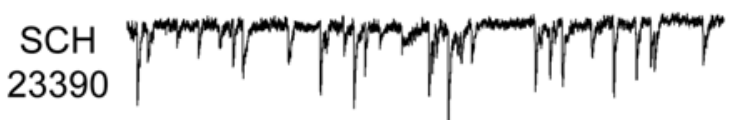

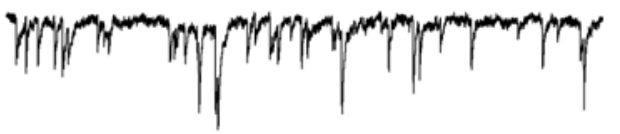
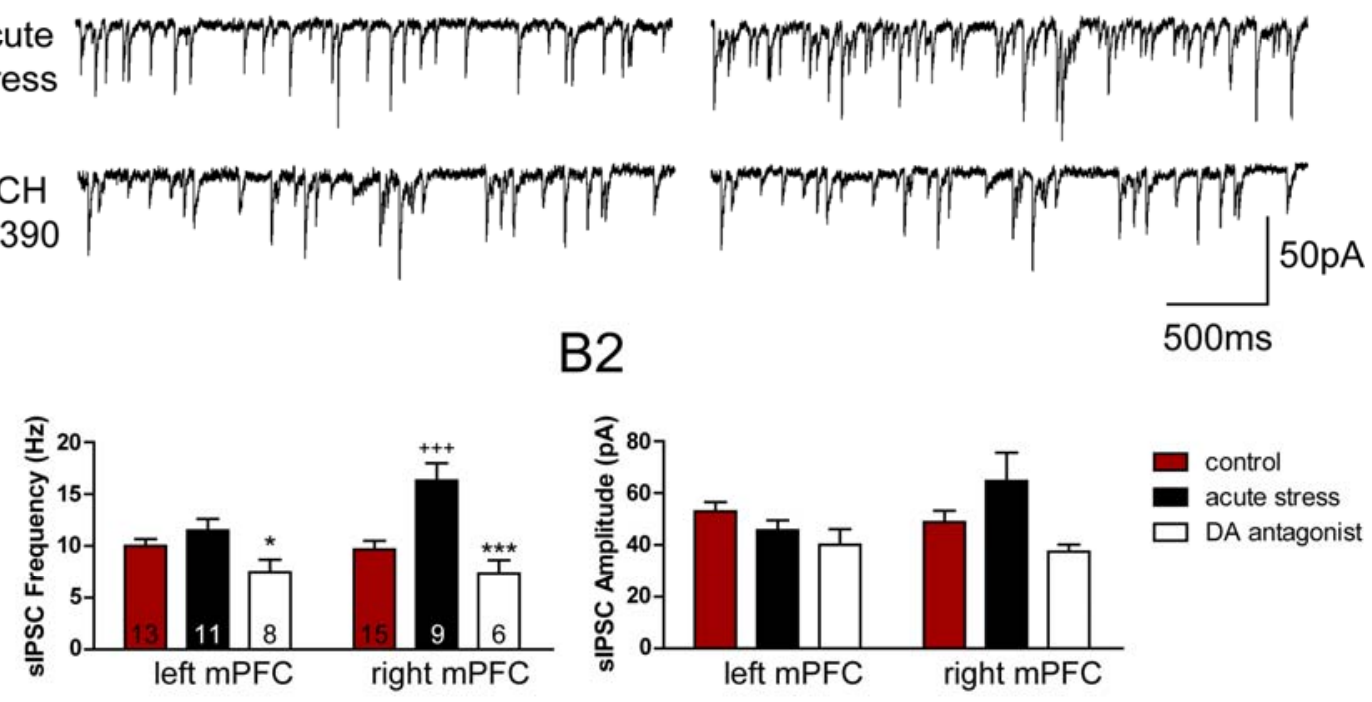

Figure 14. Lateralized acute stress effects on the GABAergic transmission in the mPFC. A: Representative recordings of sIPSCs in pyramidal cells from layer II/III mPFC. The upper and middle traces show representative recordings from a control and an acutely stressed animal, respectively. The lower traces show representative sIPSCs from an acutely stressed animal in the presence of the D1 antagonist $\mathrm{SCH}$ 23390. B: Numerical data of the means of frequency (B1) and amplitude (B2) of sIPSCs. Data are mean \pm SEM; numbers within the bars indicate the number of neurons recorded. Statistics: one-way ANOVA followed by Tukey's multiple comparison test, ${ }^{~} \mathrm{P}<0.05,{ }^{\star \star *} \mathrm{P}<0.001$, vs acute stress, ${ }^{+++} \mathrm{P}<0.001$, vs control. 


\subsubsection{Lateralized acute stress effects on sensitivity to DSI}

Because endocannabinoids significantly contribute to the presynaptic modulation at GABAergic synapses in the cerebral cortex, we designed the second experiment on the mPFC to examine whether acute stress affected CB1-mediated activity. As in the hippocampus, CB1 receptor mediated activity was assessed by analyzing the sensitivity to DSI. In the brain slices of the control rats, DSI could not be initiated in both the right and the left mPFC (Fig. 15A1,A2 upper traces,B1,B3). After 30 min of acute stress, sIPSCs recorded from pyramidal cells in the right mPFC were still DSI resistant with the normalized 5 s-averaged sIPSC frequency immediately after depolarization $104.4 \pm 4.8 \%\left(\mathrm{t}_{8}=0.9094\right.$, n.s, paired t-test; Fig. 15A1 lower trace,B3). By contrast, in the left hemisphere, a single 10 s-depolarizing voltage step induced a significant reduction in sIPSC frequency (Fig. 15A2 middle trace, B1). The averaged sIPSC frequency of the first $5 \mathrm{~s}$ after depolarization was $55.5 \pm 6.8 \%$ of the level before depolarization $\left(t_{11}=6.517, P<0.0001\right.$, paired $t$-test; Fig. 15 B1). Repeated two-way ANOVA (stress $\times$ time) revealed a significant main effect of acute stress $(F$ $=10.74, P=0.003 ;$ Fig. 15B1). The suppression of inhibition was brief and $30 \mathrm{~s}$ after depolarization, the normalized 5 s-averaged sIPSC frequency recovered to $78.2 \pm 8.9 \%$ $\left(\mathrm{t}_{11}=2.438, P<0.05\right.$, paired t-test; Fig. 15B1). Furthermore, the acute stress induced DSI in the left mPFC was completely blocked by 15 min incubation of the slices with SCH 23390 (Fig. 15A2 lower trace, B2). In the presence of SCH 23390, the normalized 5s-averaged sIPSC frequency immediately following depolarization was $106.7 \pm 16.1 \%\left(t_{5}=0.4154\right.$, n.s, paired t-test; Fig. 15B2). These data indicate that following $30 \mathrm{~min}$ of acute restraint stress, the GABAergic activities switch from DSI resistant to DSI sensitive, specifically in the left but not in the right mPFC and this effect of acute stress is also associated with an altered dopaminergic modulation. 
A1

right $m P F C$

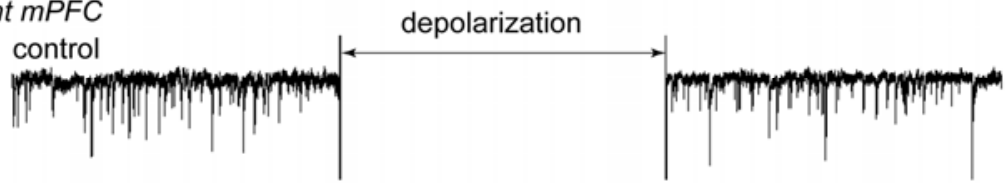

acute stress

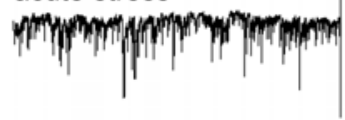

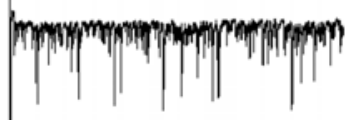

A2
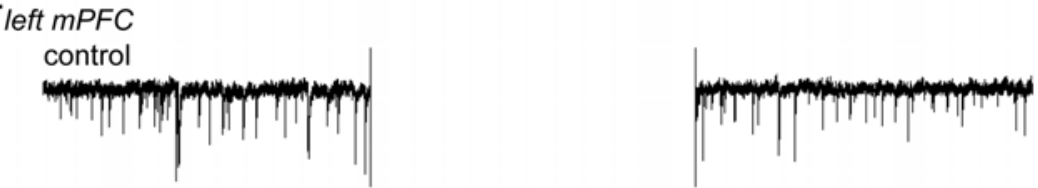

acute stress
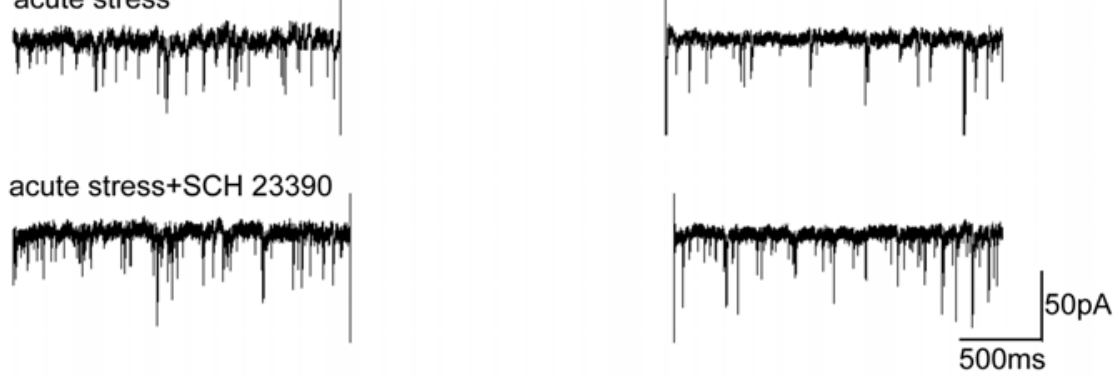

left mPFC

B1

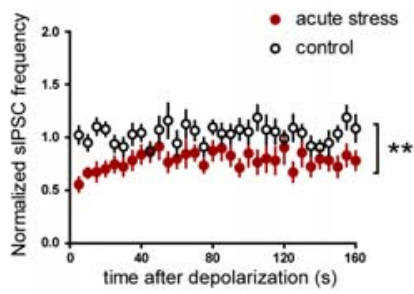

B2

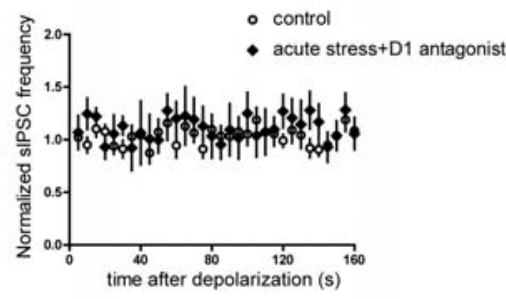

right $\mathrm{mPFC}$

B3

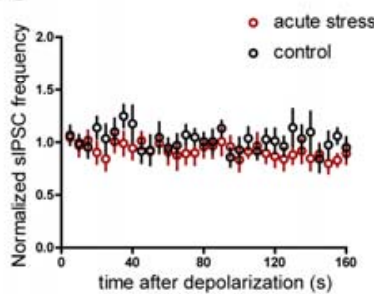

Figure 15. Lateralized effects of acute stress on sensitivity to DSI. A:

Representative recordings of DSI in pyramidal cells from layer II/III mPFC. B: Time course of DSI (5-s bin; graphs show relative changes of sIPSC frequency after depolarization). B1: 30 min of acute stress evoked switch from DSI resistant to DSI sensitive in the left mPFC (12 cells in each animal group). B2: The effect of acute stress was abolished in the presence of SCH 23390 ( 6 cells). B3: DSI resistance in the right mPFC was unafffect by acute stress (11 cells in the control group; 9 cells in the acute stress group). Repeated two-way ANOVA (stress $\times$ time), ${ }^{* *} \mathrm{P}<0.01$. 


\subsubsection{Effects of varying dopamine concentrations on GABAergic transmission and DSI sensitivity in the MPFC}

To further investigate whether dopaminergic modulation may be involved in the stress-induced lateralization of the MPFC with respect to activities of the interneuron network, a series of experiments were designed to test whether bath application of dopamine could mimic the observed stress effects.

First, effects of 100nM dopamine (low concentration) were examined. The result showed that bath application of dopamine rapidly and significantly enhanced SIPSC frequency in both right and left mPFC. The ratio between sIPSC frequency after dopamine and that before dopamine was $178.1 \pm 18.3 \%\left(t_{4}=4.266, P<0.05\right.$, paired $t$-test $)$ in the right $\mathrm{mPFC}$, and $157.3 \pm$ $10.9 \%\left(\mathrm{t}_{4}=5.273, P=0.006\right.$, paired $\mathrm{t}$-test $)$ in the left mPFC (Fig. 16A1,B). The neuronal response to a low dose of dopamine in the right and left mPFC exhibited no significant difference ( $\mathrm{t}_{8}=0.9744, n . s$, unpaired t-test). On the other hand, $100 \mathrm{nM}$ dopamine produced no effect on DSI, and sIPSCs recorded in both MPFC were still resistant to DSI. The normalized 5s-averaged sIPSC frequency immediately following 10s-depolarizing voltage step was $88.9 \pm 5.4 \%\left(t_{5}=2.060\right.$, n.s, paired $t$-test $)$ in the right $\mathrm{mPFC}$ and $117.1 \pm 11.27 \%\left(\mathrm{t}_{5}=1.517\right.$, n.s, paired t-test) in the left mPFC (Fig. 17A middle trace, B).

It is well known that the modulatory influence of dopamine on cognitive functions in the prefrontal cortex is concentration dependent, and exhibits an inverted-U shape dose-response curve (for review Arnsten, 2009). Therefore we asked the question whether a similar pattern of action also occurred in the MPFC with respect to regulation of GABAergic transmission. To test this, effects of dopamine with a higher concentration (500nM) were examined. Interestingly, bath application of 500nM dopamine produced no effect on the frequency of SIPSCs recorded from both right and left MPFC. The relative sIPSC frequency after acute stress compared to the control condition was $95.29 \pm 2.16 \%\left(t_{5}=2.182\right.$, n.s, paired $\mathrm{t}$-test) in the right mPFC and $120.4 \pm 10.07 \%\left(\mathrm{t}_{6}=2.027, n . s\right.$, paired $\mathrm{t}$-test $)$ in the left mPFC (Fig. $16 \mathrm{~A} 2, \mathrm{~B})$. Surprisingly, contrary to the situation with low dose of dopamine, in the presence of high dose of dopamine, sIPSCs in both hemispheres changed from DSI resistant to DSI sensitive. The normalized 5s-averaged SIPSC frequency immediately following 
depolarization was $48.71 \pm 9.06 \%\left(\mathrm{t}_{6}=5.657, P=0.0013\right.$, paired $\mathrm{t}$-test $)$ in the right $\mathrm{mPFC}$ and $50.22 \pm 5.24 \%\left(\mathrm{t}_{6}=9.506, P<0.0001\right.$, paired $\mathrm{t}$-test $)$ in the left mPFC (Fig. 17A lower trace, C). Repeated two-way ANOVA (drug $\times$ time) revealed a significant main effect of a high dopamine dose (right mPFC: $F=11.68, P=0.0035$; left $m P F C: F=14.55, P=0.0014$ ). In summary, these results demonstrate that a low concentration of dopamine mimics the effect of acute stress on sIPSC frequency that was observed specifically in the right mPFC (Fig. 14, Fig. 16). In contrast, a high concentration of dopamine mimics the acute stress induced DSI sensitivity observed in the left mPFC (Fig. 15, Fig 17).

A1

control

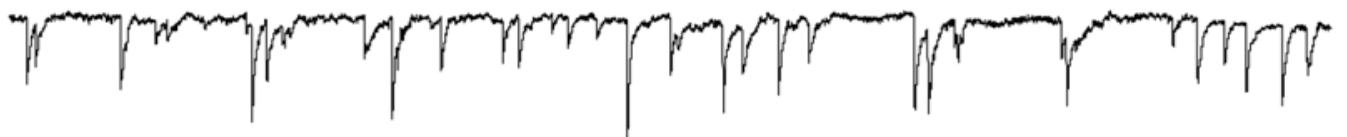

dopamine 100nM

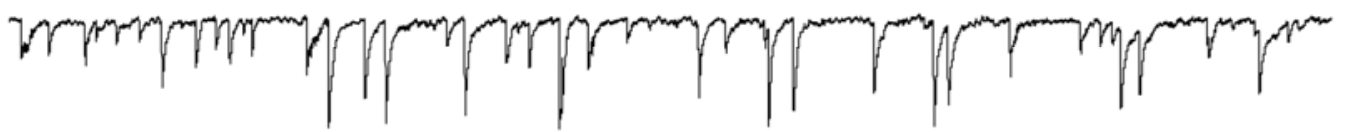

A2

control

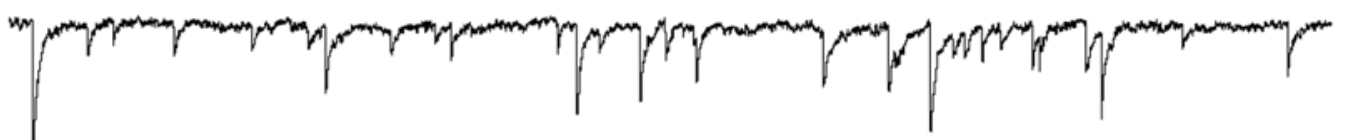

dopamine 500nM

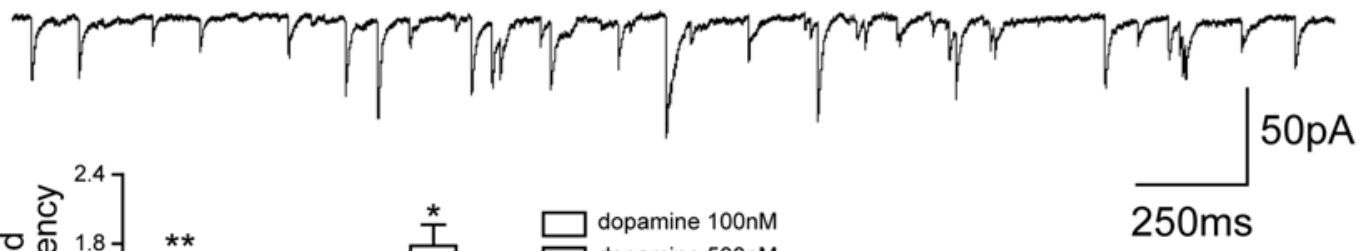

B

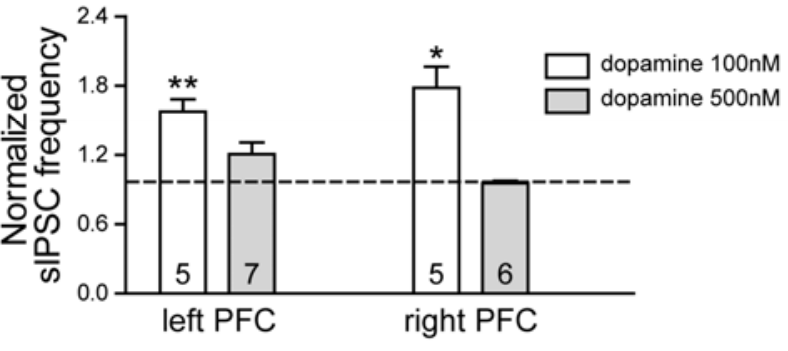

$250 \mathrm{~ms}$

Figure 16. Effects of varying dopamine concentrations on GABAergic transmission in the mPFC. A1: Representative recordings of sIPSCs in a pyramidal cell of layer II/III mPFC before and after the application of 100nM dopamine. A2: Representative recordings of sIPSCs in a pyramidal cell of layer II/III mPFC before and after the application of 500nM dopamine. B: Numerical data of normalized sIPSC frequency. Data are mean \pm SEM; means were calculated as ratio of sIPSC frequency after to that before dopamine application; numbers within the bars indicate the number of neurons recorded. Two-tailed paired t-test, ${ }^{\star} \mathrm{P}<0.05,{ }^{* \star} \mathrm{P}<0.01$. 
A

control depolarization

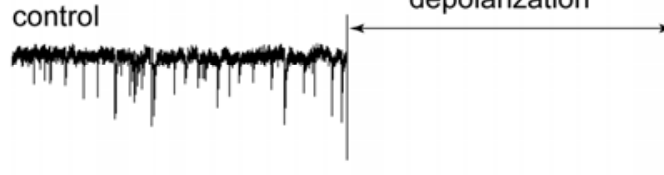

dopamine $100 \mathrm{nM}$
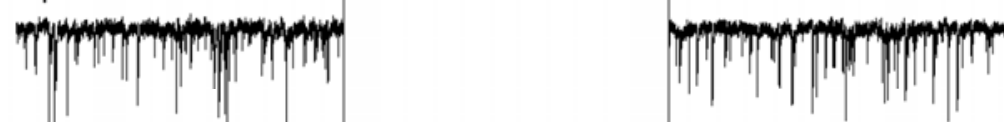

dopamine $500 \mathrm{nM}$
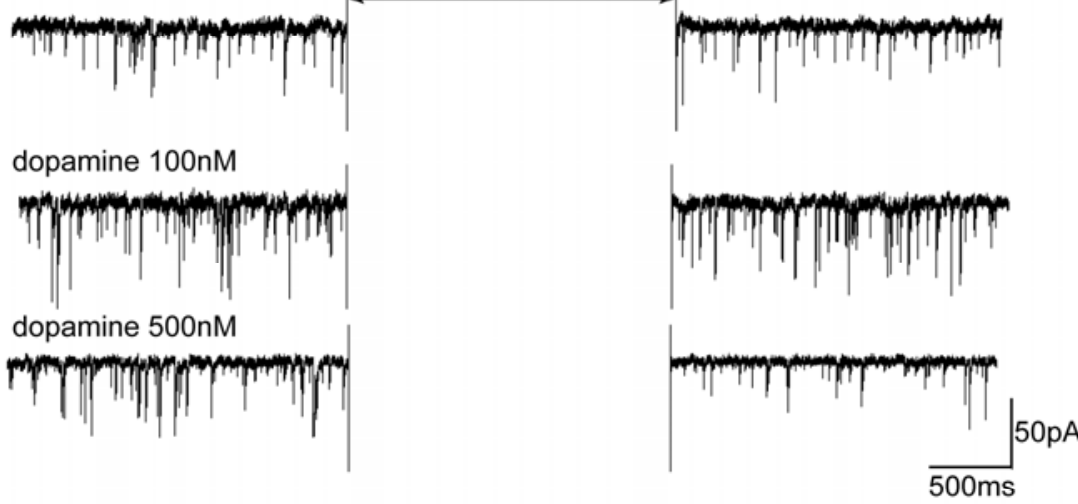

\section{left mPFC}

B1

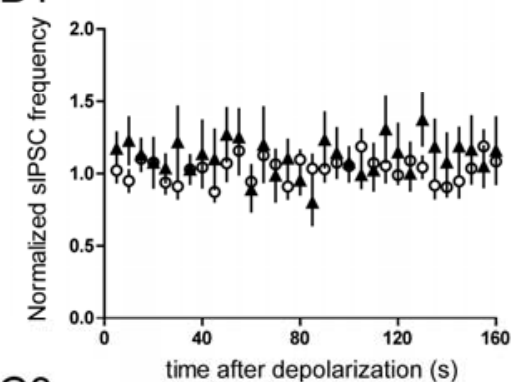

C3

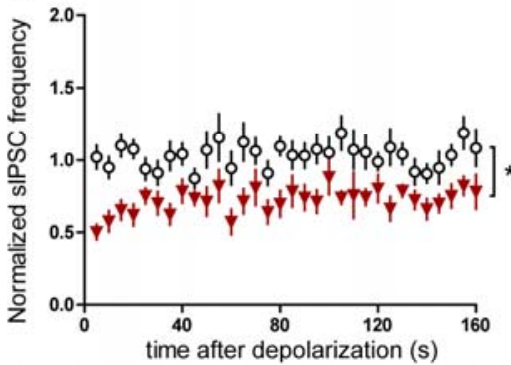

right $m P F C$

B2

- control

A dopamine $100 \mathrm{nM}$

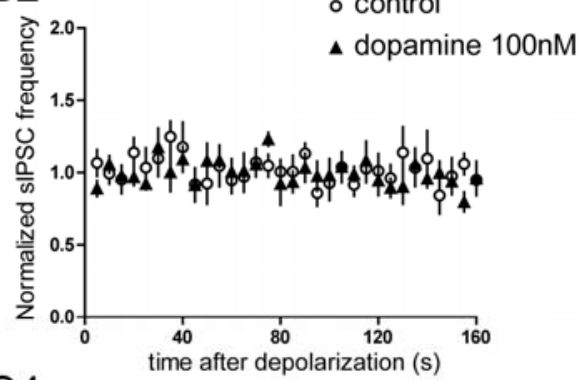

C4

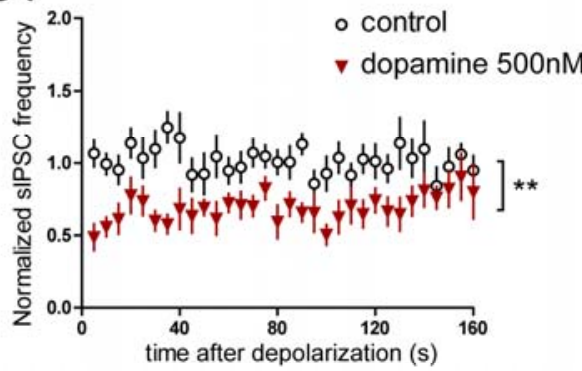

Figure 17. Effects of varying dopamine concentrations on DSI sensitivity in the mPFC. A: Representative recordings of DSI in pyramidal cells of layer II/III mPFC. B, C: Time course of DSI (5-s bin; graphs show relative changes of SIPSC frequency after depolarization). B: DSI resistance was unaffected in the presence of $100 \mathrm{nM}$ dopamine (6 cells in the left mPFC and 8 cells in the right mPFC were treated). C: In the presence of $500 \mathrm{nM}$ dopamine, sIPSCs in both hemispheres changed from DSI resistant to DSI sensitive ( 7 cells in the left $\mathrm{mPFC}$ and 7 cells in the right mPFC were treated). Repeated two-way ANOVA (drug $\times$ time), ${ }^{* *} \mathrm{P}<0.01$. 


\section{Discussion}

\subsection{Part I. The effects of GR stimulation as well as stress in the hippocampus}

The present thesis provides four novel findings: 1) acute application of a glucocorticoid receptor (GR) agonist rapidly elicits an increase in sIPSCs in the hippocampal CA1 pyramidal neurons, notably via a membrane bound GR and by retrograde nitric oxide (NO) signaling; 2) exposing animals to acute stress causes a similar enhancement of hippocampal sIPSCs; 3 ) chronic stress also results in elevated GABAergic transmission in the hippocampus which is $\mathrm{Ca}^{2+}$-dependent, and DEX elicits no further increase in sIPSCs; 4) chronic stress impairs the generation of rhythmic spontaneous IPSCs originating specifically from PV interneurons.

Given the pivotal role of perisomatic inhibition in synchronizing activities of pyramidal neurons, chronic stress-induced deficits of the PV interneurons to generate rhythmic firing may result in disturbed network oscillations and may thus contribute to cognitive impairments that are often observed in patients with stress-related psychiatric disorders. An association between the functional deficit of PV interneurons and the altered gamma oscillation has been suggested as a mechanism underlying working memory deficits in schizophrenic patients (Lewis et al., 2005; Lodge et al., 2009).

\subsubsection{Facilitation of GABAergic transmission by nongenomic glucocorticoid receptors}

Stress activates the HPA axis to increase release of corticosteroids from the adrenal glands. The cellular effects of corticosteroids are mediated chiefly via slow genomic mechanisms (De Kloet et al., 1998; Maggio and Segal, 2009). However recently, the existence of a rapid, nongenomic pathway gained substantial credit (Campolongo et al., 2009; Di et al., 2005; 2009; Haller et al., 2008; Karst et al., 2005b; Olijslagers et al., 2008). In the hypothalamus, corticosterone elicits rapid opposing effects on glutamate and GABA release of magnocellular neurons through retrograde endocannabinoid and nitric oxide signaling via divergent nongenomic pathways (Di et al., 2005; 2009). In the basolateral nucleus of the amygdala, 
corticosterone was suggested to activate $\mathrm{G}_{\mathrm{s}}$-associated membrane receptors and to induce endocannabinoid release which eventually facilitates the consolidation of aversive memories (Campolongo et al., 2009). In the hippocampus, corticosterone can boost glutamate release and promote neuronal excitability via a nongenomic MR pathway (Karst et al., 2005b; Olijslagers et al., 2008). To the best of our knowledge, we are the first to provide functional evidence for the presence of membrane bound GRs in the hippocampus. We show here that acute application of DEX elicits a surprisingly rapid facilitation of GABAergic transmission in the hippocampus of the control animals. This facilitatory action of DEX is mediated by a membrane bound receptor since the membrane impermeable BSA-DEX retained the effect. In addition we present evidence that activation of nongenomic GRs induces downstream retrograde NO signaling which eventually augments GABAergic transmission from presynaptic interneurons. The involvement of NO in modulating GABAergic transmission is supported by the studies demonstrating that hippocampal GABAergic synapses possess the molecular machinery for retrograde NO signaling (Szabadits et al., 2007). Furthermore, NO signaling has been reported to facilitate action potentials in the peptidergic neurons of the posterior pituitary (Klyachko et al., 2001). It is tempting to speculate that NO signaling induced by nongenomic GR action in the hippocampus also modulates opening of ion channels involved in certain phase(s) of an action potential to thus facilitate firing activities of the hippocampal interneurons; therefore in our experiment, action potential independent mIPSCs, which were recorded in the presence of the potent blocker of voltage-gated sodium channel TTX, were not affected by DEX. Taken together, our results suggest that activation of nongenomic GRs, by evoking retrograde NO signaling, facilitates the firing activity of the hippocampal interneurons. Given the association between GABAergic inhibition and network oscillation, the crosstalk between glucocorticoids and NO signaling may play a role in boosting oscillations to enhance cortical circuit performance in stressful situations.

Furthermore, we observed that the DEX-induced facilitation of GABA release was partially blocked by the $\mathrm{CCK}_{2}$ receptor antagonist LY225910. Moreover, the facilitating effect of the NO donor SNAP on sIPSCs was also significantly reduced in the presence of LY225910. The most likely interpretation for this is that DEX induces retrograde NO signaling which activates the NO sensitive guanylyl cyclase and thus leads to cGMP production. On the one hand, 
cGMP increases GABA transmission from both PV and CCK interneurons, and on the other hand it induces CCK release from CCK interneurons. However, it remains to be elucidated how CGMP is associated with CCK release. Since activation of $\mathrm{CCK}_{2}$ receptors massively increases the activity of PV interneurons (Földy et al., 2007), the endogenous CCK should also contribute to the facilitating effect of DEX on GABA release (Fig. 4). This interpretation is in line with recent data demonstrating that stress increases cortical CCK release (Becker et al., 2008).

\subsubsection{Stress effects on GABAergic transmission in the hippocampus}

Here we show for the first time that exposing animals to acute stress modulates the GABAergic input to CA1 pyramidal neurons. The effect of acute stress on the occurrence of sIPSCs was partially mimicked by DEX application to control slices. Both acute stress and DEX increased the frequency of sIPSCs to a similar extent, whereas the amplitude of sIPSC was affected only by DEX. In addition, in our ex vivo experimental condition, the DEX-induced facilitation of SIPSCs appeared to be relatively brief and started to decay already $\sim 10 \mathrm{~min}$ after the initial exposure to the agonist, whereas animals of the acute stress group were restrained for $30 \mathrm{~min}$ before sacrificed for brain slice preparation. The similarity and diversity between the effect of DEX and acute stress are not surprising. On the one hand, corticosterone is regarded as the main stress hormone in rodents; on the other hand, a stress response involves release of various other "stress mediators" that act in concert along with corticosterone. In addition, Maggio and Segal (2009) recently described a slow stimulatory effect of DEX on hippocampal sIPSCs which was proposed to be mediated through genomic mechanisms. It is possible that an acute stress response collectively involves fast non-genomic GR action as elucidated in the present study, as well as slow genomic GR actions which could emerge after approximately $30 \mathrm{~min}$ of stress exposure.

Furthermore, we provide the first electrophysiological evidence that the altered hippocampal GABAergic transmission sustains following a prolonged period of stress. Three weeks of daily restraint stress induced an elevation in $\mathrm{Ca}^{2+}$-dependent SIPSC frequency, and the membrane 
permeable $\mathrm{Ca}^{2+}$ chelator EGTA-AM was able to compensate this stress effect. This is in line with previous findings that chronic stress and glucocorticoid treatments regulate expression of hippocampal glutamic acid decarboxylase (GAD) which presumably leads to enhanced GABA synthesis and hippocampal GABA receptor expression (Bowers et al., 1998; Stone et al., 2001). One may argue that the observed increased activity of interneurons in the stressed animals may be due to an increased excitatory drive to interneurons rather than an effect on interneurons themselves since enhanced excitatory activity evoked by chronic stress has long been reported (Joëls et al., 2007; Joëls, 2008). However, this should not take credit for the observations under our experimental condition where glutamatergic transmission was blocked by CNQX and APV in the bath to isolate inhibitory postsynaptic currents. The increased SIPSC frequency in chronically stressed animals may indicate an increased discharge of interneurons or an enhanced release probability at their terminals.

Importantly, the same concentration of DEX that elicited rapid facilitation of sIPSCs in the control hippocampal slices failed to induce any further increase in sIPSCs in the slices from the chronically stressed animals. This result can be explained by two possibilities. (i) The rapid nongenomic DEX-NO signaling pathway is continually activated during the entire period of chronic stress, which may contribute to the $\mathrm{Ca}^{2+}$ dependent enhancement of SIPSC frequency observed in the hippocampus of the stressed animals. It is likely that the ligand binding domains of all the membrane bound GRs are saturated due to a sustained increase of GC levels so that bath application of DEX failed to induce any further effect. Or alternatively (ii), the DEX-NO signaling is destructed or desensitized after a certain period of daily stress exposures. However, during the time when this pathway is still active, sustained hyperactivity and enhanced $\mathrm{Ca}^{2+}$ influx into the interneurons might induce activity-dependent gene transcription and progressively result in secondary adaptive alterations in expression levels or properties of proteins that are associated with GABA metabolism and/or transmission (e.g. GAD) and/or ion channels that regulate the firing activity of the interneurons (e.g. voltage-gated calcium channel). These secondary changes may underlie the observed chronic stress-induced elevation in hippocampal GABAergic transmission. 


\subsubsection{A functional deficit of the PV+ neuronal network induced by chronic stress}

As discussed above endogenous CCK is released in response to GC exposure. Since CCK specifically stimulates PV interneurons (Földy et al., 2007) it appears that during stress, stimulatory effects converge on the PV interneurons in that they are affected by both NO and CCK. Being in this hub site, PV interneurons are probably in a more vulnerable position compared to CCK interneurons. In support of this hypothesis, an earlier morphometric study suggested that PV interneurons in the hippocampus are the one vulnerable to stress (Czéh et al., 2005). Coinciding with this, our present results show that after three weeks of restraint stress the number of PV-immunoreactive cells was significantly reduced in all hippocampal subfields. Furthermore, the PV interneuron network of the stressed animals exhibited a functional deficit in that it failed to generate rhythmic sIPSCs in response to the CCK analog. One may argue that the functional deficit of the PV interneuron network reflects a dysfunction of $\mathrm{CCK}_{2}$ receptors. At this point we can not rule out this possibility, however, bath application of CCK induced a massive increase of sIPSCs in both the control and the chronic stress group. It was only the rhythmicity of the CCK-induced SIPSCs that was impaired by chronic stress. On the other hand, application of EGTA-AM could normalize the chronic stress-induced elevation in the hippocampal SIPSC frequency, suggesting that the stress-stimulated GABA transmission comes along with excessive intracellular free $\mathrm{Ca}^{2+}$. As reported earlier, an elaborately regulated $\mathrm{Ca}^{2+}$ signaling contributes to the synchronous release of GABA quanta from hippocampal PV interneurons (Caillard et al., 2000; Hefft and Jonas, 2005). Therefore, we hypothesize that during chronic stress, the prolonged elevation of corticosterone results in sustained high activity of PV interneurons which in turn leads to increased $\mathrm{Ca}^{2+}$ influx that is beyond the buffering capacity of the calcium binding protein PV expressed in those neurons. As a consequence, the high presynaptic $\mathrm{Ca}^{2+}$ level on the one hand boosts GABA release from the PV interneurons and on the other hand results in impaired temporal precision and inaccurately timed response patterns of these neurons. 


\subsubsection{Summary: The nongenomic DEX action and the effects of chronic stress on the PV interneuron network}

The summary of the above findings and a theoretical explanation are presented in Fig. 18. The data demonstrate that short-term exposure to DEX increases activity of hippocampal interneurons through retrograde signaling of $\mathrm{NO}$ and $\mathrm{CCK}$ (downstream from $\mathrm{NO}$ ) by activating membrane bound GRs in the pyramidal neurons. We suggest that the outlined nongenomic action of DEX provides a potential pathogenic mechanism which underlies the chronic stress-induced effects on the PV interneuron network reported in the present thesis, although the cellular mechanisms of acute glucocorticoid action and the chronic stress effects are likely to differ. We hypothesize that the continual activation of nongenomic GRs contributes to the development of the chronic stress-induced $\mathrm{Ca}^{2+}$ dependent elevation in GABAergic transmission. Furthermore, since hippocampal PV interneurons are affected by both NO and CCK their excessive excitation may eventually develop into a cellular and functional deficit. The PV interneuron network plays a vital role in the regulation of synchronized activities (Somogyi and Klausberger, 2005; Sohal et al., 2009) and, as suggested by Freund and Katona (2007), operates as clockworks for cortical network oscillations. Oscillatory brain activity has been proposed to provide a temporal structure for information processing and thereby contributes to cognitive functions such as memory formation (Buzsáki and Chrobak, 1995). Therefore, the stress-induced structural and functional deficits of the PV interneurons may contribute to the altered oscillation patterns that are implicated in cognitive impairments which are commonly observed in animal models of chronic stress as well as in patients with stress-related psychiatric illnesses. 

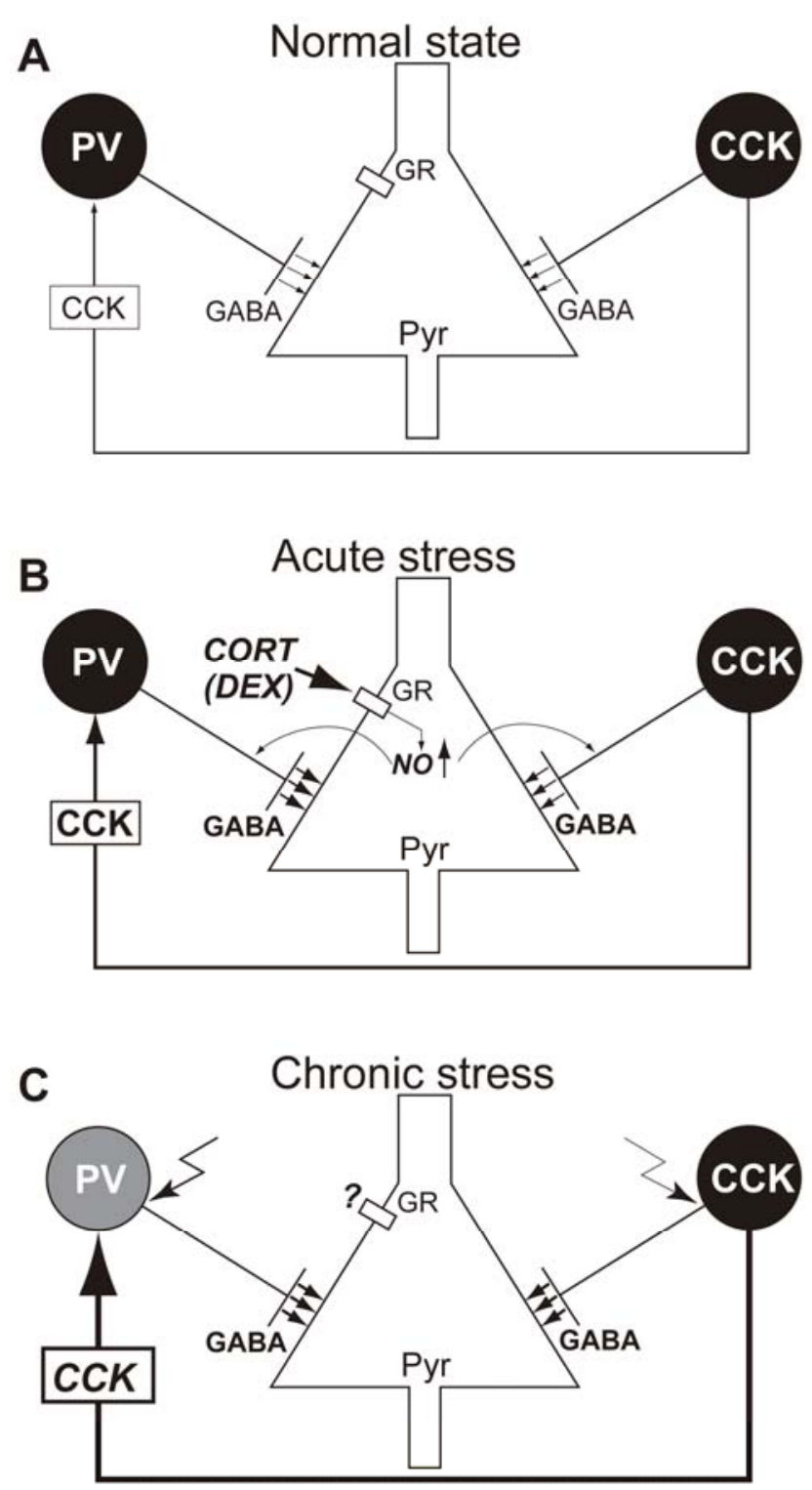

Figure 18. Summary: the nongenomic DEX action and the effects of chronic stress on the PV interneuron network. A: Under control conditions, $\mathrm{PV}+$ and $\mathrm{CCK}+$ cells provide two parallel, functionally distinct sources of perisomatic GABAergic inhibition to the pyramidal cells. In addition, CCK, presumably released from the $\mathrm{CCK}+$ cells, stimulates the $\mathrm{PV}+$ cells. B: In response to acute stress, the glucocorticoid surge rapidly activates membrane bound GRs on pyramidal cells which results in NO release. Retrograde NO signaling stimulates GABA release from the GABAergic terminals. In addition, NO induces release of CCK which in turn stimulates PV+ cells. C: During chronic stress, the above mechanisms are continuously or repetitively activated which results in sustained elevation of $\mathrm{Ca}^{2+}$ influx and GABA release. In this situation, the $\mathrm{PV}+$ cells are in a more vulnerable position as they receive stimulatory effects also from the CCK+ cells. The persistently elevated $\mathrm{Ca}^{2+}$ level in the $\mathrm{PV}+$ cells is detrimental to their cellular integrity, and their capacity to generate rhythmic oscillations is impaired. Thus, chronic stress causes functional and structural deficits in $\mathrm{PV}+$ cells. Secondary adaptive alterations of proteins associated with GABAergic transmission are probably induced to facilitate sustained hyperactivity of interneurons. CCK: cholecystokinin; GR: glucocorticoid receptor; NO: nitric oxide; PV: parvalbumin; Pyr: pyramidal neuron. Modified from Hu et al., 2010. 


\subsubsection{Effects of chronic stress on endocannabinoid mediated suppression of GABA release from CCK interneurons}

We demonstrate here that chronic stress impairs the endocannabinoid mediated modulation of GABAergic transmission in the hippocampus which is consistent with previous reports showing that chronic stress downregulates expression of hippocampal CB1 receptors which are expressed almost exclusively on CCK expressing interneurons (Hill et al., 2005). As described earlier, the CCK interneuron activity is subject to modulation by DSI, a widespread form of short-term synaptic plasticity. The impairment of DSI following chronic stress suggests an altered functional integrity of the CCK interneuron network even though the ability of the network to generate rhythmic activity in response to exogenous stimuli remains intact.

Previous physiological studies documented alterations in CB1 activity in the striatum and amygdala following chronic stress. Downregulation of CB1 receptor expression in the hippocampus by chronic stress has long been reported, and coincides with the present data which provide neurophysiological evidence that endocannabinoid mediated control of GABAergic transmission in the hippocampus is impaired. However, it is still largely unknown how it contributes to behavioral effects of chronic stress. It was reported that chronic stress induces impairment in hippocampus-dependent cognitive tasks which could be reversed by administration of exogeneous cannabinoids, indicating a role of the endocannabinoid system in cognition. Conversely, electrophysiolgical evidence suggested that endocannabinoid signaling, by inducing DSI, may play a role in hippocampal encoding of information in that it allows local pyramidal cells to escape oscillatory entrainment to thus become more sensitive to LTP induction (Carlson et al., 2002). Therefore the impaired endocannabinoid-mediated modulation of the CCK interneuron activity presented here provides further insight into the neuroplastic changes that may underlie the cognitive deficits that are commonly observed following chronic stress.

Effects of environmental stress on endocannabinoid signaling are apparently complex and brain region dependent, and they vary with the duration as well as the context of the stress paradigm. A cross talk between endocannabinoids and glucocorticoids which are increased 
upon stress exposure has been reported for hypothalamus and amygdala (Di et al, 2005; Campolongo et al, 2009). This cross talk may contribute to enhanced endocannabinoid activity following stress within these brain regions (Patel et al., 2009; Rademacher et al., 2008). Interestingly, it was reported that impairment of CB1 mediated signaling in the striatum which was induced by chronic social defeat stress could be prevented by pharmacological blockade of glucocorticoid receptors and was mimicked by chronic administration of a glucocorticoid agonist (Rossi et al., 2008). However, how interactions between endocannabinoids and glucocorticoids occur in the hippocampus and to which extent they contribute to the chronic stress induced disruption of hippocampal endocannabinoid signaling is still to be investigated. 


\subsection{Part II: Stress effects in the mPFC}

\subsubsection{A chronic stress-induced reduction in the number of PV-immunoreactive cells in} the right $\mathrm{mPFC}$

The data from the experiments described in this thesis indicate that the total number of PV interneurons in the mPFC of the adult male rat is modulated by chronic stress in a hemisphere specific manner. This reduction in the number of PV interneurons observed in the right mPFC might be indicative of cell death. However, to the best of my knowledge, chronic stress-induced apoptosis in the mPFC has so far not been demonstrated. Alternatively, because of the stress insult, the contents of perikaryal $\mathrm{PV}$ within individual interneurons may fall below levels detectable by immunocytochemistry. A reduction in the calcium-binding protein PV may potentially reflect a functional alteration in the interneurons (Caillard et al., 2000; Müller et al., 2007). Additional physiological analyses are necessary to further elucidate what mechanisms may underlie this effect of chronic stress, and how the reduction in the number of $\mathrm{PV}$ interneurons in the right mPFC may affect functioning of the neuronal network in the area.

\subsubsection{Lateralized effects of acute stress on the interneuron network within the mPFC}

The present thesis provides data from preliminary experiments indicating that acute stress differentially regulates network activity of GABAergic interneurons in the left and the right mPFC respectively. In the right mPFC, the frequency of sIPSCs as well as mIPSCs increased following $30 \mathrm{~min}$ of restraint stress. In contrast in the left mPFC, the same parameters remained unaltered; however, sIPSCs underwent a transition from DSI resistance to DSI sensitivity in the left mPFC, indicating that acute stress sensitized endocannabinoid signaling in this hemisphere.

The acute stress effects in the mPFC could be abolished by blocking receptors of the D1 family, suggesting that dopaminergic modulation is associated with the alterations in the activity of interneuron network following acute stress. Furthermore, the acute stress effects 
observed in the present study were mimicked by dopamine in a dose dependent manner, with a low concentration of the catecholamine mimicking the stress effect observed in the right mPFC and higher concentration mimicking that in the left mPFC. Conversely, it was previously shown that restraint stress induced a lateralized increase in dopamine release in the mPFC with a brief exposure of about 30 min resulting in a pronounced dopamine release in the left mPFC (Carlson et al., 1991). In view of the findings that dopamine per se (100 and $500 \mathrm{nM}$ ) produced no lateralized effects in the present study, we propose that the hemispheric difference in dopamine release upon acute stress is responsible for the lateralized alterations in the functioning of interneurons in the mPFC.

Notably, in our in vitro experimental condition, the differences in the dopamine concentrations in brain slices from control and stressed rats were probably minimal because of the processing of the slices during preparation. Therefore, it seems unlikely that the hemispheric differences in response to acute stress detected in the present study were directly due to different concentrations in left and right mPFC in the slices. However, one may speculate that in vivo, the increase in dopaminergic activity evoked by stress in either hemisphere results in some secondary posttranslational modifications, changes in surface expression of the dopamine receptors or alterations in signaling pathways linked to those receptors. Further investigations are necessary to confirm the present findings, to elucidate which changes occur upon acute stress and whether they contribute to the observed alterations in activities of interneurons in the mPFC.

\subsubsection{Dose-dependent actions of dopamine}

The present study indicates that a low dose of dopamine increases sIPSC frequency in the mPFC. By contrast, a high dose of dopamine induces sensitivity to DSI while sIPSC frequency is unaffected under this condition. The complexity of the dose dependent actions of dopamine indicates that different interneuron subtypes are involved. In rodent PFC, it has been shown that the PV-expressing interneurons, which constitute a major subpopulation of fast-spiking interneurons (Kawaguchi and Kubota, 1997), receive more efficient excitatory 
input from and make significantly more inhibitory synaptic contacts onto local pyramidal cells than the other interneuron subtypes such as the irregular-spiking ones (Galarreta et al., 2008). In addition, it has been reported that in the rat PFC, the high affinity dopamine D5 receptors (affinity of dopamine for D5 is 10-fold higher than for D1) are located on the somata and dendrites of PV interneurons and in pyramidal cells (Oda et al., 2010). Both D1 and D5 receptors, which belong to the D1-like family (D1Rs), are associated with $G_{s}$ and thus stimulate adenylyl cyclase activity upon activation (Seamans and Yang, 2004; Trantham-Davidson et al., 2004). On the other hand, it was reported that CB1 receptors in the rat mPFC are present on calbindin (CBL) but not on PV or calretinin-expressing GABAergic interneurons (Wedzony and Chocyk et al., 2009). Because of this CB1 expression, GABAergic input originating from $\mathrm{CBL}$ interneurons is presumably sensitive to endocannabinoid mediated DSI.

Taken together, the interpretation of the observed dopamine effects in the MPFC is presented as follows:

1) When a low dose of dopamine $(100 \mathrm{nM})$ is applied to the mPFC slices, the high affinity D5 receptors are activated. This leads to enhanced activity of the PV interneuron network in the $\mathrm{mPFC}$, which is manifested by the increase in SIPSC frequency in both hemispheres (Fig. 16). Coinciding with this view, it was reported previously that dopamine increased the excitability of fast-spiking interneurons in rat PFC via activation of D1Rs (Gorelova et al., 2002). Because the majority of GABAergic input is provided by the PV interneurons which do not express the endocannabinoid CB1 receptors (Galarreta et al., 2008; Wedzony and Chocyk et al., 2009), the sIPSCs recorded at this experimental condition are DSI resistant (Fig. 17B, Fig. 19B).

2) Bath application of a higher dose of dopamine (500nM) switches the recorded sIPSCs from DSI resistant to DSI sensitive, which is mediated by endocannabinoid signaling (Fig. 17C). This indicates that dopamine at this concentration evokes an increased proportion of GABAergic input that is sensitive to endocannabinoids. This component of GABAergic input originates from the $\mathrm{CB} 1$-expressing $\mathrm{CBL}$ interneurons. Therefore, this result suggests that a higher dose of dopamine evokes enhanced activity of the CBL interneurons in the mPFC (Fig. 19C). 
3) Bath application of 500nM dopamine produced no significant effect on SIPSC frequency. One may speculate that $500 \mathrm{nM}$ dopamine simultaneously activates both D1Rs (associated with $\mathrm{G}_{\mathrm{s}}$ and thus stimulating adenylyl cyclase activity upon activation) and D2Rs (associated with $G_{i}$, inhibition of adenylyl cyclase activity) so that their opposing cellular effects might counterbalance the neuronal reaction. However, it was reported previously that activation of D2Rs requires even higher concentrations of dopamine $(>1 \mu \mathrm{M}$, Trantham-Davidson et al., 2004). Alternatively, it has been reported that the CBL interneurons in the hippocampus provide GABAergic input to other interneuron subtypes, beside principal cells (Vida et al., 1998). We speculate that this may also be the case for the $\mathrm{CBL}$ interneuron in the $\mathrm{MPFC}$ and the PV interneuron may constitute one of its postsynaptic targets. Thus, the enhanced firing of the CBL interneurons may counteract the D5-mediated activation of the PV interneurons (Fig. 16, Fig. 19C). This could explain why the overall sIPSCs received by the pyramidal cells are unaltered in the presence of the higher dopamine dose.

However, which receptor subtype of the D1R family is responsible for the increased firing activity of the $\mathrm{CBL}$ interneuron is yet to be clarified. A possible explanation is that the $\mathrm{CBL}$ interneurons in the mPFC express the low affinity D1 receptors which also present in the mPFC (Ariano and Sibley, 1994); D1 receptors are stimulated by dopamine at higher concentrations. Further investigations should be designed to test this using receptor subtype-specific antibodies or probes that can distinguish between D1 and D5 receptors. Unfortunately, currently subtype-specific agonists/antagonists are still commercially unavailable and therefore to test this model with pharmacological approaches is difficult. 
A Normal physiological condition in right and left mPFC

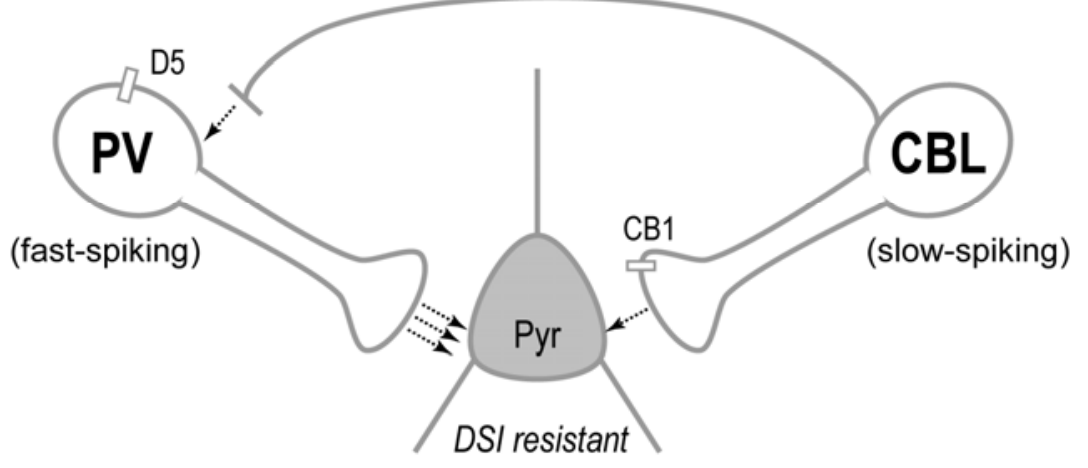

B
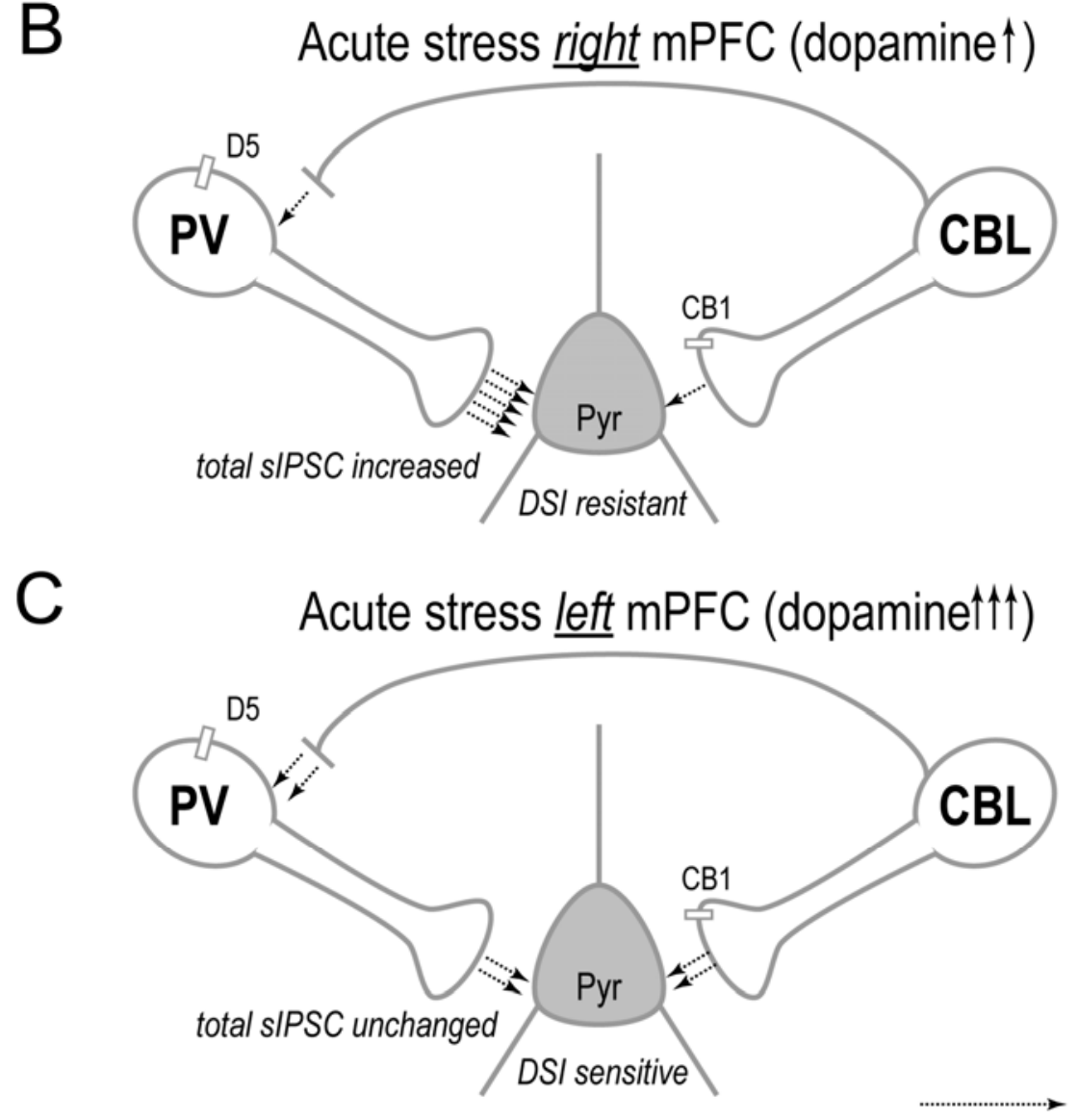

GABA release

Figure 19. Summary of the preliminary data on the modulation of interneuron network in the mPFC by lateralized dopamine release in response to acute stress. A: Under normal physiological conditions, PV and $C B L$ interneurons provide two functionally distinct sources of GABAergic inhibition to pyramidal cells in the mPFC. B,C: Acute stress leads to increased dopamine release in the mPFC with more pronounced change in the left hemisphere. $\mathrm{B}$ : In the right $\mathrm{mPFC}$, the lower concentration of dopamine may lead to an elevated firing activity of fast-spiking PV interneurons by activating D5 receptors. As a result, GABAergic transmission is increased in the right mPFC. C: In the left mPFC, the high concentration of dopamine may activate $\mathrm{CB} 1$ expressing $\mathrm{CBL}$ interneurons. Increase in the proportion of input from $\mathrm{CBL}$ interneurons switches GABAergic transmission from DSI resistant to DSI sensitive. Meanwhile, increased inhibitory input from CBL interneurons to PV interneurons may counteract the D5-mediated activation of the PV interneurons. As a result, total GABAergic transmission is unchanged in the left mPFC. CB1: endocannabinoid receptor; CBL: calbindin; D5: dopamine D5 receptor; PV: parvalbumine; Pyr: Pyramidal neuron 


\subsubsection{Lateralized dopamine release and acute stress response}

Lateralized dopamine release in response to stress using various stress paradigms and different stress durations has long been reported (Carlson et al, 1991; 1993; Sullivan, 2004). The present results suggest a novel neuronal mechanism (summarized in Fig. 19) that could explain how this lateralized release contributes to the functional changes in the mPFC during stress. As discussed earlier (chapter 4.2.2 and 4.2.3), an acute stress-induced hemispheric asymmetry in dopamine release may contribute to the lateralized functional changes of interneurons in the mPFC. The lower stress-induced dopamine release in the right mPFC may result in an elevated GABAergic transmission by increasing firing activity of fast-spiking PV interneurons (Fig. 19B). By contrast in the left mPFC, the high stress-induced dopamine release may activate inhibitory innervation from CB1 expressing CBL interneurons. Increase in the proportion of input from CBL interneurons in turn switches the total GABAergic transmission from DSI resistant to DSI sensitive (Fig. 19C). It has been shown in the neocortex, that networks of different interneuron subtypes promote different patterns of coordinated activities (Freund 2003; Klausberger et al. 2005; Somogyi and Klausberger 2005). Therefore, the present data indicate that lateralized alterations in dopamine release upon a brief stress exposure differentially change the interneuron networks in the mPFC hemispheres. Furthermore, the potentially lateralized functional shift to either PV or CBL interneurons may significantly contribute to lateralized functions of mPFC in regulation of the stress response. However, further experiments are required to confirm and verify this theory.

\subsubsection{Presumptive functional implication}

Sullivan (2004) proposed a theory of the lateralized function of the mPFC in stress regulation. According to this theory, when an animal is exposed to an environmental challenge, the immediate response is the activation of the left PFC which is less emotional and more motor dominant than the right hemisphere and thus may be more suited for regulation of efficient coping with the stressor. However, when stress is prolonged or uncontrollable and coping attempts fail, the right PFC will become dominant. Additionally, the author suggested that the 
mesocortical dopamine pathway which innervates both PFC hemispheres plays a protective role against the pathological effects of stress, although each may be preferentially associated with distinct stages of coping with stressful experiences (Sullivan, 2004).

The effects of acute restraint stress in the mPFC shown in the present thesis are consistent with this theory. The acute stress induced enhancement in the GABAergic inhibitory input from fast-spiking PV interneurons to layer II/III pyramidal neurons in the right mPFC probably suppresses firing of pyramidal neurons and may result in reduced activity of the right mPFC. By contrast, the total inhibitory input in the left mPFC was unaffected by acute stress. On the other hand, it has been proposed that D5 receptor activation in the mPFC enhances glutamatergic excitatory transmission (Oda et al., 2010). Therefore in the left mPFC, where inhibitory GABAergic transmission is unaffected as revealed in the present study, the net effect of stress-induced dopamine release is probably an enhanced activity of this hemisphere. Furthermore, the present results show that acute stress leads to a sensitized endocannabinoid signaling in the left mPFC. The significance of this effect on functions of the $\mathrm{mPFC}$ in stress regulation is still to be determined. There have been evidences indicate that the endocannabinoid system modulates complex behavioral responses, such as escaping (Patel et al., 2005) and sensitivity to natural reward (Rademacher and Hillard, 2007) both of which are important for dealing with stress. It is likely that the enhanced signaling of the endocannabinoid system upon acute stress is also involved in the initial coping that is, as suggested in Sullivan's theory, executed in the left PFC.

\subsection{Summary and conclusions}

The present thesis provides many novel findings regarding the effects of acute as well as chronic stress on GABAergic transmission within the hippocampal formation and the medial prefrontal cortex. The results reveal the increased hippocampal GABAergic transmission following acute as well as chronic restraint stress. In addition, we find that prolonged stress results in impaired temporal precision in the activity of the PV interneurons and impaired endocannabinoid modulation of the CCK interneuron activity in the hippocampal formation. I 
suggest that these alterations in functional integrity of both interneuron networks collectively contribute to cognitive deficits which are commonly observed in animal models of stress as well as in patients with stress-related psychiatric disorders. On the other hand, the hippocampal PV and CCK interneuron are recruited at different times during network activity and they are proposed to play different functional roles in perisomatic inhibition (Glickfeld and Scanziani, 2006; Freund and Katona, 2007). Therefore, additional analyses are required to further differentiate between the functional significance of the reported stress induced alterations in the network of PV and CCK interneurons.

Moreover, a new nongenomic glucocorticoid receptor signaling pathway in the hippocampus is identified in the present study, which may represent a potential pathogenic mechanism underlying the chronic stress-induced effects on the PV interneuron network. These findings have the potential to further improve the understanding of the etiology of the stress related psychiatric diseases.

Preliminary experiments also indicate that in the medial prefrontal cortex, acute stress leads to a lateralized functional shift in the interneuron network activity. Further investigations suggest that a differential dopaminergic modulation in the right versus the left mPFC, probably due to lateralized dopamine release, may contribute to these stress effects. These results suggest a novel physiological mechanism underlying the neuronal basis for coping with stress. However, further studies are required to confirm the present findings. 


\section{References}

Abumaria N, Rygula R, Hiemke C, Fuchs E, Havemann-Reinecke U, Rüther E, Flügge G (2007) Effect of chronic citalopram on serotonin-related and stress-regulated genes in the dorsal raphe nucleus of the rat. Eur Neuropsychopharmacol. 17:417-29.

Akirav I, Maroun M (2007) The role of the medial prefrontal cortex-amygdala circuit in stress effects on the extinction of fear. Neural Plast 2007:30873.

Alfonso J, Frasch AC, Flügge G (2005) Chronic stress, depression and antidepressants: effects on gene transcription in the hippocampus. Rev Neurosci 16:43-56.

Amat J, Baratta MV, Paul E, Bland ST, Watkins LR, Maier SF (2005) Medial prefrontal cortex determines how stressor controllability affects behavior and dorsal raphe nucleus. Nat Neurosci 8:365-371.

Ariano MA, Sibley DR (1994) Dopamine receptor distribution in the rat CNS: elucidation using anti-peptide antisera directed against D1A and D3 subtypes. Brain Res 649:95-110.

Arnsten AF (2009) Stress signalling pathways that impair prefrontal cortex structure and function. Nat Rev Neurosci 10:410-22.

Becker C, Zeau B, Rivat C, Blugeot A, Hamon M, Benoliel JJ (2008) Repeated social defeat-induced depression-like behavioral and biological alterations in rats: involvement of cholecystokinin. Mol Psychiatry 13:1079-92.

Bowers G, Cullinan WE, Herman JP (1998) Region-specific regulation of glutamic acid decarboxylase (GAD) mRNA expression in central stress circuits. J Neurosci 18:5938-47.

Bremner JD (2002). Neuroimaging studies in post-traumatic stress disorder. Curr Psychiatry Rep 4: 254-263. 
Brambilla P, Perez J, Barale F, Schettini G, Soares JC (2003) GABAergic dysfunction in mood disorders. Mol Psychiatry 8:721-37, 715.

Buzsáki G (2002) Theta oscillations in the hippocampus. Neuron 33:325-40.

Buzsáki G, Chrobak JJ (1995) Temporal structure in spatially organized neuronal ensembles: a role for interneuronal networks. Curr. Opin. Neurobiol. 5:504-510.

Buzsáki G, Draguhn A (2004) Neuronal oscillations in cortical networks. Science 304:1926-9.

Caillard O, Moreno H, Schwaller B, Llano I, Celio MR, Marty A (2000) Role of the calcium-binding protein parvalbumin in short-term synaptic plasticity. Proc Natl Acad Sci U S A 97:13372-7.

Campolongo P, Roozendaal B, Trezza V, Hauer D, Schelling G, McGaugh JL, et al. (2009) Endocannabinoids in the rat basolateral amygdala enhance memory consolidation: Involvement of the glucocorticoid system. Proc Natl Acad Sci U S A 106:4888-93.

Caspi A, Sugden K, Moffitt TE, Taylor A, Craig IW, Harrington H, McClay J, Mill J, Martin J, Braithwaite A, Poulton R (2003) Influence of life stress on depression: Moderation by a polymorphism in the 5-HTT gene. Science 301:386-389.

Carlson G, Wang Y, and Alger BE (2002) Endocannabinoids facilitate the induction of LTP in the hippocampus. Nat Neurosci 5: 723-724.

Carlson JN, Fitzgerald LW, Keller Jr RW, Glick SD (1991) Side and region dependent changes in dopamine activation with various durations of restraint stress. Brain Res $550: 313-8$.

Carlson JN, Fitzgerald LW, Keller Jr RW, Glick SD (1993) Lateralized changes in prefrontal cortical dopamine activity induced by controllable and uncontrollable stress in the rat. Brain Res 630:178-87. 
Conboy L, Sandi C (2010) Stress at learning facilitates memory formation by regulating AMPA receptor trafficking through a glucocorticoid action. Neuropsychopharmacology 35:674-85.

Cruz-Orive LM, Weibel ER (1990) Recent stereological methods for cell a brief survey. Am. J. Physiol. 258:148-156.

Czéh B, Michaelis T, Watanabe T, Frahm J, de Biurrun G, van Kampen M, Bartolomucci A, Fuchs E (2001). Stress-induced changes in cerebral metabolites, hippocampal volume, and cell proliferation are prevented by antidepressant treatment with tianeptine. Proc Natl Acad Sci U S A 98:12796-12801.

Czéh B, Simon M, van der Hart MG, Schmelting B, Hesselink MB, Fuchs E (2005) Chronic stress decreases the number of parvalbumin-immunoreactive interneurons in the hippocampus: prevention by treatment with a substance $P$ receptor (NK1) antagonist. Neuropsychopharmacology 30:67-79.

Czéh B, Perez-Cruz C, Fuchs E, Flügge G (2008) Chronic stress-induced cellular changes in the medial prefrontal cortex and their potential clinical implications: does hemisphere location matter? Behav Brain Res. 190:1-13.

Conrad CD, Galea LA, Kuroda Y, McEwen BS. (1996) Chronic stress impairs rat spatial memory on the $\mathrm{Y}$ maze, and this effect is blocked by tianeptine pretreatment. Behav Neurosci 110:1321-34.

Conrad CD, LeDoux JE, Magariños AM, McEwen BS (1999) Repeated restraint stress facilitates fear conditioning independently of causing hippocampal CA3 dendritic atrophy. Behav Neurosci 113:902-13.

de Groote L, Linthorst AC (2007) Exposure to novelty and forced swimming evoke stressor-dependent changes in extracellular GABA in the rat hippocampus. Neuroscience 148:794-805. 
de Kloet ER, Vreugdenhil E, Oitzl MS, Joëls M (1998) Brain corticosteroid receptor balance in health and disease. Endocr Rev 19:269-301.

de Kloet ER, Joëls M, Holsboer F (2005) Stress and the brain: from adaptation to disease. Nat Rev Neurosci 6:463-75.

DeFelipe J (1997) Types of neurons, synaptic connections and chemical characteristics of cells immunoreactive for calbindin-D28K, parvalbumin and calretinin in the neocortex. $\mathrm{J}$ Chem Neuroanat 14:1-19.

Di S, Malcher-Lopes R, Marcheselli VL, Bazan NG, Tasker JG (2005) Rapid glucocorticoid-mediated endocannabinoid release and opposing regulation of glutamate and gamma-aminobutyric acid inputs to hypothalamic magnocellular neurons. Endocrinology $146: 4292-301$.

Di S, Maxson MM, Franco A, Tasker JG (2009) Glucocorticoids regulate glutamate and GABA synapse-specific retrograde transmission via divergent nongenomic signaling pathways. J Neurosci 29:393-401.

Diorio D, Viau V, Meaney MJ (1993) The role of the medial prefrontal cortex (cingulated gyrus) in the regulation of hypothalamic-pituitary-adrenal responses to stress. J Neurosci 13: 3839-3847.

Drevets WC, Price JL, Furey ML (2008) Brain structural and functional abnormalities in mood disorders: implications for neurocircuitry models of depression. Brain Struct Funct 213:93-118.

Duma D, Jewell CM, Cidlowski JA (2006) Multiple glucocorticoid receptor isoforms and mechanisms of post-translational modification. J Steroid Biochem Mol Biol. 102:11-21.

Euston DR, Tatsuno M, McNaughton BL (2007) Fast-forward playback of recent memory sequences in prefrontal cortex during sleep. Science 318:1147-50. 
Fava M, Kendler KS (2000). Major depressive disorder. Neuron 28:335-341.

Férézou I, Cauli B, Hill EL, Rossier J, Hamel E, Lambolez B (2002) 5-HT3 receptors mediate serotonergic fast synaptic excitation of neocortical vasoactive intestinal peptide/cholecystokinin interneurons. J Neurosci 22:7389-97.

Földy C, Lee SY, Szabadics J, Neu A, Soltesz I (2007) Cell type-specific gating of perisomatic inhibition by cholecystokinin. Nat Neurosci 10:1128-30

Freedman R, Wetmore C, Strömberg I, Leonard S, Olson L (1993) Alpha-bungarotoxin binding to hippocampal interneurons: immunocytochemical characterization and effects on growth factor expression. J Neurosci 13:1965-75.

Freund TF, Katona I (2003) Perisomatic Inhibition. Neuron. 56:33-42.

Fride E, Suris R, Weidenfeld J, Mechoulam R (2005) Differential response to acute and repeated stress in cannabinoid CB1 receptor knockout newborn and adult mice. Behav Pharmacol 16:431-440.

Fuchs E, Czéh B, Flügge G (2004a). Examining novel concepts of the pathophysiology of depression in the chronic psychosocial stress paradigm in tree shrews. Behav Pharmacol 15:315-325.

Fuchs E, Czéh B, Kole MH, Michaelis T, Lucassen PJ (2004b). Alterations of neuroplasticity in depression: the hippocampus and beyond. Eur Neuropsychopharmacol 14 Suppl 5:S481-S490.

Gabbott PL, Dickie BG, Vaid RR, Headlam AJ, Bacon SJ (1997) Local-circuit neurones in the medial prefrontal cortex (areas 25, 32 and 24b) in the rat: morphology and quantitative distribution. J Comp Neurol 377:465-499.

Galarreta M, Erdélyi F, Szabó G, Hestrin S (2008) Cannabinoid sensitivity and synaptic properties of 2 GABAergic networks in the neocortex. Cereb Cortex 18:2296-305. 
Ge S, Pradhan DA, Ming GL, Song H (2007) GABA sets the tempo for activity-dependent adult neurogenesis. Trends Neurosci 30:1-8.

Glickfeld LL, Scanziani M (2006) Distinct timing in the activity of cannabinoid-sensitive and cannabinoid-insensitive basket cells. Nat Neurosci 9:807-15.

Goldman-Rakic PS, Muly EC 3rd, Williams GV (2000) D(1) receptors in prefrontal cells and circuits. Brain Res Rev 31:295-301.

Gomez,F, Houshyar,H, Dallman,MF (2002) Marked regulatory shifts in gonadal, adrenal, and metabolic system responses to repeated restraint stress occur within a 3-week period in pubertal male rats. Endocrinology 143: 2852-2862.

Gorelova N, Seamans JK, Yang CR (2002) Mechanisms of dopamine activation of fast-spiking interneurons that exert inhibition in rat prefrontal cortex. J Neurophysiol 88:3150-66.

Gratton A, Sullivan RM (2005) Role of prefrontal cortex in stress responsivity. In: Steckler T, Kalin NH, Reul JMHM, editors. Techniques in the behavioral and neural sciences, no. 1, vol. 15. Amsterdam: Elsevier p. 807-17.

Gundersen HJ, Bagger P, Bendtsen TF, Evans SM, Korbo L, Marcussen N, et al. (1988). The new stereological tools: disector, fractionator, nucleator and point sampled intercepts and their use in pathological research and diagnosis. APMIS 96: 857-881.

Hájos N, Katona I, Naiem SS, MacKie K, Ledent C, Mody I, Freund TF (2000) Cannabinoids inhibit hippocampal GABAergic transmission and network oscillations. Eur J Neurosci 12:3239-49.

Haller J, Mikics E, Makara GB (2008) The effects of nongenomic glucocorticoid mechanisms on bodily functions and the central neural system. A critical evaluation of findings. Front Neuroendocrinol 29:273-91. 
Hasler G, van der Veen JW, Tumonis T, Meyers N, Shen J, Drevets WC (2007) Reduced prefrontal glutamate/glutamine and gamma-aminobutyric acid levels in major depression determined using proton magnetic resonance spectroscopy. Arch Gen Psychiatry 64:193-200.

Hebb AL, Poulin JF, Roach SP, Zacharko RM, Drolet G (2005) Cholecystokinin and endogenous opioid peptides: interactive influence on pain, cognition, and emotion. Prog Neuropsychopharmacol Biol Psychiatry 29:1225-38.

Hefft S, Jonas P (2005) Asynchronous GABA release generates long-lasting inhibition at a hippocampal interneuron-principal neuron synapse. Nat Neurosci 8:1319-28.

Herman JP, Figueiredo H, Mueller NK, Ulrich-Lai Y, Ostrander MM, Choi DC, Cullinan WE (2003) Central mechanisms of stress integration: hierarchical circuitry controlling hypothalamo-pituitary-adrenocortical responsiveness. Front Neuroendocrinol 24:151-80.

Hill MN, Hunter RG, McEwen BS (2009) Chronic stress differentially regulates cannabinoid CB1 receptor binding in distinct hippocampal subfields. Eur J Pharmacol. 614: 66-9.

Hill MN, Patel S, Carrier EJ, Rademacher DJ, Ormerod BK, Hillard CJ, Gorzalka BB (2005) Downregulation of endocannabinoid signaling in the hippocampus following chronic unpredictable stress. Neuropsychopharmacology 30: $508-515$.

Howard MW, Rizzuto DS, Caplan JB, Madsen JR, Lisman J, Aschenbrenner-Scheibe R, Schulze-Bonhage A, Kahana MJ (2003) Gamma oscillations correlate with working memory load in humans. Cereb Cortex 13:1369-74.

Joëls M, Karst H, Krugers HJ, Lucassen PJ (2007) Chronic stress: implications for neuronal morphology, function and neurogenesis. Front Neuroendocrinol 28:72-96.

Joëls M (2008) Functional actions of corticosteroids in the hippocampus. Eur J Pharmacol 583:312-21. Review. 
Juhasz G, Chase D, Pegg E, Downey D, Toth ZG, Stones K, et al. (2009). CNR1 gene is associated with high neuroticism and low agreeableness and interacts with recent negative life events to predict current depressive symptoms. Neuropsychopharmacology 34: 2019-2027.

Karst H, Joëls M (2003) Effect of chronic stress on synaptic currents in rat hippocampal dentate gyrus neurons. J Neurophysiol 89:625-33.

Karst H, Joëls M (2005a) Corticosterone slowly enhances miniature excitatory postsynaptic current amplitude in mice CA1 hippocampal cells. J Neurophysiol. 94:3479-86.

Karst H, Berger S, Turiault M, Tronche F, Schütz G, Joëls M (2005b) Mineralocorticoid receptors are indispensable for nongenomic modulation of hippocampal glutamate transmission by corticosterone. Proc Natl Acad Sci USA 102:19204-7.

Karson MA, Whittington KC, Alger BE (2008) Cholecystokinin inhibits endocannabinoid-sensitive hippocampal IPSPs and stimulates others. Neuropharmacology $54: 117-128$.

Kawaguchi Y, Kubota Y (1997) GABAergic cell subtypes and their synaptic connections in rat frontal cortex. Cereb Cortex 7:476-86.

Kendler KS, Karkowski LM, Prescott CA (1999). Causal relationship between stressful life events and the onset of major depression. Am J Psychiatry 156:837-841.

Keuker JI, Vollmann-Honsdorf GK, Fuchs E (2001) How to use the optical fractionator: an example based on the estimation of neurons in the hippocampal CA1 and CA3 regions of tree shrews. Brain Res Brain Res Protoc 7:211-21.

Klausberger T, Marton LF, O'Neill J, Huck JH, Dalezios Y, Fuentealba P, et al. (2005) Complementary roles of cholecystokinin- and parvalbumin-expressing GABAergic neurons in hippocampal network oscillations. J Neurosci 25:9782-93. 
Klyachko VA, Ahern GP, Jackson MB (2001) cGMP-mediated facilitation in nerve terminals by enhancement of the spike afterhyperpolarization. Neuron 31: 1015-25.

Kole MH, Costoli T, Koolhaas JM, Fuchs E (2004) Bidirectional shift in the cornu ammonis 3 pyramidal dendritic organization following brief stress. Neuroscience 125:337-47.

Krystal JH, Sanacora G, Blumberg H, Anand A, Charney DS, Marek G, et al. (2002) Glutamate and GABA systems as targets for novel antidepressant and mood-stabilizing treatments. Mol Psychiatry 7:S71-80.

Lee T, Jarome T, Li SJ, Kim JJ, Helmstetter FJ (2009) Chronic stress selectively reduces hippocampal volume in rats: a longitudinal magnetic resonance imaging study. Neuroreport 20:1554-8.

Lewis DA, Hashimoto T, Volk DW (2005) Cortical inhibitory neurons and schizophrenia. Nat Rev Neurosci 6:312-24.

Liu RJ, Aghajanian GK (2008) Stress blunts serotonin- and hypocretin-evoked EPSCs in prefrontal cortex: role of corticosterone-mediated apical dendritic atrophy. Proc Natl Acad Sci U S A 105:359-64.

Lodge DJ, Behrens MM, Grace AA (2009) A loss of parvalbumin-containing interneurons is associated with diminished oscillatory activity in an animal model of schizophrenia. $\mathrm{J}$ Neurosci 29:2344-54.

Luine V, Villegas M, Martinez C, McEwen BS (1994) Stress-dependent impairments of spatial memory. Role of 5-HT. Ann N Y Acad Sci. 746:403-4.

MacQueen GM, Campbell S, McEwen BS, Macdonald K, Amano S, Joffe RT, et al. (2003). Course of illness, hippocampal function, and hippocampal volume in major depression. Proc Natl Acad Sci USA 100:1387-1392.

Maejima T, Ohno-Shosaku T, Kano M (2001) Endogenous cannabinoid as a retrograde 
messenger from depolarized postsynaptic neurons to presynaptic terminals. Neurosci Res 40:205-10. Review.

Magariños AM, McEwen BS (1995) Stress-induced atrophy of apical dendrites of hippocampal CA3c neurons: involvement of glucocorticoid secretion and excitatory amino acid receptors. Neuroscience 69: 89-98.

Magariños AM, McEwen BS, Flügge G, Fuchs E (1996) Chronic psychosocial stress causes apical dendritic atrophy of hippocampal CA3 pyramidal neurons in subordinate tree shrews. J Neurosci 16:3534-40.

Magariños AM, Verdugo JM, McEwen BS (1997) Chronic stress alters synaptic terminal structure in hippocampus. Proc Natl Acad Sci U S A 94:14002-8.

Maggio N, Segal M (2009) Differential corticosteroid modulation of inhibitory synaptic currents in the dorsal and ventral hippocampus. J Neurosci 29:2857-66.

Mamalaki E, Kvetnansky R, Brady LS, Gold PW, Herkenham M (1992) Repeated immobilization stress alters tyrosine hydroxylase, corticotropin-releasing hormone and corticosteroid receptor messenger ribonucleic acid levels in rat brain. $\mathrm{J}$ Neuroendocrinol 4:689- 99 .

Marais L, Stein DJ, Daniels WM (2009) Exercise increases BDNF levels in the striatum and decreases depressive-like behavior in chronically stressed rats. Metab Brain Dis. 24:587-97.

Markram H, Toledo-Rodriguez M, Wang Y, Gupta A, Silberberg G, Wu C (2004) Interneurons of the neocortical inhibitory system. Nat Rev Neurosci 5:793-807.

Mason JW (1968a) A review of psychoneuroendocrine research on the pituitary-adrenal-cortical system. Psychosom Med 30:576-609.

Mason JW (1968b) A review of psychoendocrine research on the sympathetic-adrenal-medullary system. Psychosom Med 30:631-53. 
Mathers CD, Loncar D (2006). Projections of global mortality and burden of disease from 2002 to 2030. PLoS Med 3:e442.

McBain CJ, Fisahn A (2001) Interneurons unbound. Nat Rev Neurosci 2:11-23.

McEwen BS, De Kloet ER, Rostene W (1986) Adrenal steroid receptors and actions in the nervous system. Physiol Rev 66: 1121-88.

McEwen BS (2007) Physiology and neurobiology of stress and adaptation: central role of the brain. Physiol Rev 87:873-904.

McLaughlin KJ, Gomez JL, Baran SE, Conrad CD (2007) The effects of chronic stress on hippocampal morphology and function: an evaluation of chronic restraint paradigms. Brain Res 1161:56-64.

Megías M, Emri Z, Freund TF, Gulyás Al (2001) Total number and distribution of inhibitory and excitatory synapses on hippocampal CA1 pyramidal cells. Neuroscience 102:527-40.

Meyer U, van Kampen M, Isovich E, Flugge G, Fuchs E (2001) Chronic psychosocial stress regulates the expression of both GR and MR mRNA in the hippocampal formation. Hippocampus 11:329- 36 .

McLaughlin KJ, Gomez JL, Baran SE, Conrad CD (2007) The effects of chronic stress on hippocampal morphology and function: an evaluation of chronic restraint paradigms. Brain Res 1161:56-64.

Milad MR, Quirk GJ (2002) Neurons in medial prefrontal cortex signal memory for fear extinction. Nature 420:70-4.

Miles R, Toth K, Gulyas Al, Hajos N, Freund TF (1996) Differences between somatic and dendritic inhibition in the hippocampus. Neuron 16, 815-823.

Miller EK (2000) The prefrontal cortex and cognitive control. Nat Rev Neurosci 1:59-65. 
Morales M, Bloom FE (1997) The 5-HT3 receptor is present in different subpopulations of GABAergic neurons in the rat telencephalon. J Neurosci 17:3157-67.

Morgan MA, Romanski LM, LeDoux JE (1993) Extinction of emotional learning: contribution of medial prefrontal cortex. Neurosci Lett 163:109-13.

Müller M, Felmy F, Schwaller B, Schneggenburger R (2007) Parvalbumin is a mobile presynaptic $\mathrm{Ca} 2+$ buffer in the calyx of held that accelerates the decay of $\mathrm{Ca} 2+$ and short-term facilitation. J Neurosci 27:2261-71.

Sousa N, Lukoyanov NV, Madeira MD, Almeida OF, Paula-Barbosa MM (2000) Reorganization of the morphology of hippocampal neurites and synapses after stress-induced damage correlates with behavioral improvement. Neuroscience 97:253-266.

Ohno-Shosaku T, Maejima T, Kano M (2001) Endogenous cannabinoids mediate retrograde signals from depolarized postsynaptic neurons to presynaptic terminals. Neuron 29:729-38.

Olijslagers JE, de Kloet ER, Elgersma Y, van Woerden GM, Joëls M, Karst H (2008) Rapid changes in hippocampal CA1 pyramidal cell function via pre- as well as postsynaptic membrane mineralocorticoid receptors. Eur J Neurosci 27:2542-50.

Orchinik M, Carroll SS, Li YH, McEwen BS, Weiland NG (2001) Heterogeneity of hippocampal GABA(A) receptors: regulation by corticosterone. J Neurosci 21:330-9.

Papp E, Leinekugel X, Henze DA, Lee J, Buzsáki G (2001) The apical shaft of CA1 pyramidal cells is under GABAergic interneuronal control. Neuroscience 102:715-21.

Pascucci T, Ventura R, Latagliata EC, Cabib S, Puglisi-Allegra S (2007) The medial prefrontal cortex determines the accumbens dopamine response to stress through the opposing influences of norepinephrine and dopamine. Cereb Cortex 17:2796-2804.

Pascual-Le Tallec L, Lombès M (2005) The mineralocorticoid receptor: a journey exploring its diversity and specificity of action. Mol Endocrinol 19:2211-21. 
Paskitti ME, McCreary BJ, Herman JP (2000) Stress regulation of adrenocorticosteroid receptor gene transcription and mRNA expression in rat hippocampus: time-course analysis. Brain Res Mol Brain Res 80:142-52.

Patel S, Roelke CT, Rademacher DJ, Hillard CJ (2005) Inhibition of restraint stress-induced neural and behavioural activation by endogenous cannabinoid signalling. Eur $\mathrm{J}$ Neurosci 21:1057-1069.

Patel S, Kingsley PJ, Mackie K, Marnett LJ, Winder DG (2009) Repeated homotypic stress elevates 2-arachidonoylglycerol levels and enhances short-term endocannabinoid signaling at inhibitory synapses in basolateral amygdala. Neuropsychopharmacology 34:2699-709.

Perez-Cruz C, Muller-Keuker JIH, Heilbronner U, Fuchs E, Flügge G (2007) Morphology of pyramidal neurons in the rat prefrontal cortex: lateralized dendritic remodeling by chronic stress. Neural Plasticity [Article ID 46276].

Perez-Cruz C, Simon M, Czéh B, Flügge G, Fuchs E (2009) Hemispheric differences in basilar dendrites and spines of pyramidal neurons in the rat prelimbic cortex: activity- and stress-induced changes. Eur J Neurosci 29:738-47.

Pittenger C, Duman RS (2008) Stress, depression, and neuroplasticity: a convergence of mechanisms. Neuropsychopharmacology 33:88-109.

Porter JT, Cauli B, Tsuzuki K, Lambolez B, Rossier J, Audinat E (1999) Selective excitation of subtypes of neocortical interneurons by nicotinic receptors. J Neurosci 19:5228-35.

Rademacher DJ, Hillard CJ (2007) Interactions between endocannabinoids and stress-induced decreased sensitivity to natural reward. Prog Neuropsychopharmacol Biol Psychiatry 31:633-641.

Rademacher DJ, Meier SE, Shi L, Ho WS, Jarrahian A, Hillard CJ (2008) Effects of acute and repeated restraint stress on endocannabinoid content in the amygdala, ventral striatum, and 
medial prefrontal cortex in mice. Neuropharmacology 54:108 -116.

Radley JJ, Sisti HM, Hao J, Rocher AB, McCall T, Hof PR, McEwen BS, Morrison JH (2004) Chronic behavioral stress induces apical dendritic reorganization in pyramidal neurons of the medial prefrontal cortex. Neuroscience 125:1-6.

Reich CG, Karson MA, Karnup SV, Jones LM and Alger BE (2005) Regulation of IPSP theta rhythm by muscarinic receptors and endocannabinoids in hippocampus. J Neurophysiol 94:4290-4299.

Reul JM, de Kloet ER (1985) Two receptor systems for corticosterone in rat brain: microdistribution and differential occupation. Endocrinology 117:2505-2511.

Roberts EA (1988) GABA and Benzodiazepine Receptors (Squires, R., ed.), pp. 1-21, CRC

Rossi S, De Chiara V, Musella A, Kusayanagi H, Mataluni G, Bernardi G, Usiello A, Centonze D (2008) Chronic psychoemotional stress impairs cannabinoid-receptor-mediated control of GABA transmission in the striatum. J Neurosci 28:7284-92.

Sanacora G, Mason GF, Rothman DL, Behar KL, Hyder F, Petroff OA, et al. (1999). Reduced cortical gamma-aminobutyric acid levels in depressed patients determined by proton magnetic resonance spectroscopy. Arch Gen Psychiatry 56:1043-7.

Schulte-Herbrüggen O, Fuchs E, Abumaria N, Ziegler A, Danker-Hopfe H, Hiemke C, Hellweg R (2009) Effects of escitalopram on the regulation of brain-derived neurotrophic factor and nerve growth factor protein levels in a rat model of chronic stress. J Neurosci Res $87: 2551-60$.

Seamans JK, Yang CR (2004) The principal features and mechanisms of dopamine modulation in the prefrontal cortex. Prog Neurobiol 74:1-58.

Sheline YI, Gado MH, Kraemer HC (2003). Untreated depression and hippocampal volume loss. Am J Psychiatry 160: 1516-1518. 
Sohal VS, Zhang F, Yizhar O, Deisseroth K (2009) Parvalbumin neurons and gamma rhythms enhance cortical circuit performance. Nature 459:698-702.

Somogyi P, Klausberger T (2005) Defined types of cortical interneurone structure space and spike timing in the hippocampus. J Physiol 562:9-26.

Steiner MA, Wanisch K, Monory K, Marsicano G, Borroni E, Bachli H, Holsboer F, Lutz B, Wotjak CT (2008) Impaired cannabinoid receptor type 1 signaling interferes with stress-coping behavior in mice. Pharmacogenomics J 8:196 -208.

Sterio DC (1984). The unbiased estimation of number and sizes of arbitrary particles using the disector. J Microsc 134:127-136.

Stewart MG, Davies HA, Sandi C, Kraev IV, Rogachevsky VV, Peddie CJ, et al. (2005) Stress suppresses and learning induces plasticity in CA3 of rat hippocampus: a three-dimensional ultrastructural study of thorny excrescences and their postsynaptic densities. Neuroscience 131: 43-54.

Stone DJ, Walsh JP, Sebro R, Stevens R, Pantazopolous H, Benes FM (2001) Effects of preand postnatal corticosterone exposure on the rat hippocampal GABA system. Hippocampus 11: $492-507$.

Sullivan RM, Gratton A (1999) Lateralized effects ofmedial prefrontal cortex lesions on neuroendocrine and autonomic stress responses in rats. J Neurosci 19:2834-40.

Sullivan RM, Gratton A (2002) Prefrontal cortical regulation of hypothalamic-pituitary-adrenal function in the rat and implications for psychopathology: side matters. Psychoneuroendocrinology 27:99-114.

Sullivan RM (2004) Hemispheric asymmetry in stress processing in rat prefrontal cortex and the role of mesocortical dopamine. Stress 7:131-43.

Sunahara RK, Guan HC, O'Dowd BF, Seeman P, Laurier LG, Ng G, George SR, Torchia J, 
Van Tol HH, Niznik HB (1991) Cloning of the gene for a human dopamine D5 receptor with higher affinity for dopamine than D1. Nature 350:614-619.

Szabadits E, Cserép C, Ludányi A, Katona I, Gracia-Llanes J, Freund TF, et al. (2007) Hippocampal GABAergic synapses possess the molecular machinery for retrograde nitric oxide signaling. J Neurosci 27:8101-11.

Trantham-Davidson H, Neely LC, Lavin A, Seamans JK (2004) Mechanisms underlying differential D1 versus D2 dopamine receptor regulation of inhibition in prefrontal cortex. J Neurosci 24:10652-9.

Vanderwolf $\mathrm{CH}$ (1969). Hippocampal electrical activity and voluntary movement in the rat. Electroencephalogr Clin Neurophysiol 26: 407-418.

Vetter P, Roth A, Häusser M (2001) Propagation of action potentials in dendrites depends on dendritic morphology. J Neurophysiol 85:926-37.

Vida I, Halasy K, Szinyei C, Somogyi P, Buhl EH (1998) Unitary IPSPs evoked by interneurons at the stratum radiatum-stratum lacunosum-moleculare border in the CA1 area of the rat hippocampus in vitro. J Physiol 506:755-73.

Vollmann-Honsdorf GK, Flügge G, Fuchs E (1997) Chronic psychosocial stress does not affect the number of pyramidal neurons in tree shrew hippocampus. Neurosci Lett 233:121-4.

Watanabe Y, Gould E, McEwen BS (1992) Stress induces atrophy of apical dendrites of hippocampal CA3 pyramidal neurons. Brain Res 588:341-5.

Wedzony K, Chocyk A (2009) Cannabinoid CB1 receptors in rat medial prefrontal cortex are colocalized with calbindin- but not parvalbumin- and calretinin-positive GABAergic neurons. Pharmacol Rep 61:1000-7.

Weinshank RL, Adham N, Macchi M, Olsen MA, Branchek TA, Hartig PR (1991) Molecular cloning and characterization of a high affinity dopamine receptor (D1 beta) and its 
pseudogene. J Biol Chem 266:22427-22435.

West MJ (1999). Stereological methods for estimating the total number of neurons and synapses: issues of precision and bias. Trends Neurosci 22: 51-61.

West MJ, Gundersen HJ (1990) Unbiased stereological estimation of the number of neurons in the human hippocampus, J Comp Neurol 296:1-22.

West MJ, Slomianka L, Gundersen HJ (1991). Unbiased stereological estimation of the total number of neurons in the subdivisions of the rat hippocampus using the optical fractionator. Anat Rec 231: 482-497.

Woolley CS, Gould E, McEwen BS (1990) Exposure to excess glucocorticoids alters dendritic morphology of adult hippocampal pyramidal neurons. Brain Res 531:225-31.

Yoshida T, Hashimoto K, Zimmer A, Maejima T, Araishi K, Kano M (2002) The cannabinoid CB1 receptor mediates retrograde signals for depolarization-induced suppression of inhibition in cerebellar Purkinje cells. J Neurosci 22:1690-7.

Zaitsev AV, Gonzalez-Burgos G, Povysheva NV, Kroner S, Lewis DA, Krimer LS (2005) Localization of calcium-binding proteins in physiologically and morphologically characterized interneurons of monkey dorsolateral prefrontal cortex. Cereb Cortex 15:1178-1186.

Pittenger C, Duman RS (2008) Stress, depression, and neuroplasticity: a convergence of mechanisms. Neuropsychopharmacology 33:88-109. 


\section{List of tables and figures}

\section{Tables}

Table 1: Numerical data of relative changes of carbachol-induced IPSC frequency following depolarization in CA1.

Table 2: Stereological results: Mean estimated total numbers of PV-immunoreactive interneurons in right and left mPFC after chronic restraint stress.

\section{Figures}

Figure 1: Glucocorticoid receptor stimulation facilitates GABAergic transmission. $\quad 29$

Figure 2: DEX facilitates GABAergic transmission through a nongenomic mechanism.

Figure 3: The DEX-induced facilitation of GABAergic transmission is mediated by retrograde nitric oxide signaling.

Figure 4: The DEX-induced facilitation of GABAergic transmission is partially mediated by endogenous CCK release downstream from the nitric oxide signaling. 36

Figure 5: Acute stress increases hippocampal GABAergic transmission. 37

Figure 6: Physiological parameters in response to chronic stress. 38

Figure 7: Chronic stress increases hippocampal GABAergic transmission. 40

Figure 8: Bath application of DEX (25nM) failed to elicit any significant change in sIPSCs in the hippocampus of chronically stressed rats.

Figure 9: Effects of chronic stress on the number of PV- and CCK-immunoreactive cells in the hippocampus.

Figure 10: The ability of CCK interneurons to generate rhythmic sIPSCs in response to carbachol was unaltered after chronic stress.

Figure 11: The ability of PV interneurons to generate rhythmic sIPSCs in response to CCK-8S was impaired by chronic stress. 
Figure 12: Chronic stress impairs endocannabinoid mediated suppression of CCK interneuron activity.

Figure 13: Lateralized effects of chronic stress on the number of PV-immunoreactive cells in the mPFC.

Figure 14: Lateralized acute stress effects on the GABAergic transmission in the mPFC.

Figure 15: Lateralized effects of acute stress on sensitivity to DSI.

Figure 16: Effects of varying dopamine concentrations on GABAergic transmission in the mPFC.

Figure 17: Effects of varying dopamine concentrations on DSI sensitivity in the MPFC.

Figure 18: Summary: the nongenomic DEX action and the effects of chronic stress on the PV interneuron network.

66

Figure 19: Summary: Lateralized dopamine release in response to brief stress modulates interneuron network in the right and left mPFC. 


\section{Acknowledgements}

First of all I would like to thank Prof. Gabriele Flügge for her constructive advices, constant support and enormous patience throughout my Ph.D. thesis. I am more than grateful to her for giving me a chance to work on this project and allowing me to work as independently as I could and try out my own research ideas as much as possible.

I am greatly indebted to Dr. Boldizsár Czéh. His insightful and inspiring discussions, which lasted even after he had left this group, helped a lot in guiding me during my work.

A substantial number of experiments of my thesis were performed in the lab of Prof. Weiqi Zhang, now head of the Department of Psychiatry, Westfälische Wilhelms University of Münster, and I am indebted to his generosity. His helpful suggestions during the study are indeed also acknowledged. I would like to thank Mingyue for his great and invaluable help throughout the electrophysiology experiments and for keeping me company in the lab.

I would like also to acknowledge the members of my thesis committee: Prof. Ralf Heinrich and Prof. Hubert Jarry for their supports, critical comments and suggestions.

Special thanks go out to Prof Eberhard Fuchs for giving me the opportunity to work in the group of the Clinical Neurobiology Laboratory.

I am additionally grateful to the coordination office of the MSc/PhD Neuroscience program. Their effort in all administrative matters of this excellent study program is the reason for me being here. Special gratitude goes to Prof. Michael Hörner and Dr. Steffen Burkhardt for their great job in managing all student issues during the past years.

My appreciation also goes out to Cornelia $\mathrm{H}$. for all the help with the animals and to Simone B., Simone L. and Andreas H. for their technical assistance.

Members of the Clinical Neurobiology Laboratory at the German Primate Center, Adema, Nicole, Carolina, Kerstin, Yifan and Enrique, thank you all for all the enjoyable times.

Finally I would like to thank my husband for all his love that has kept me warm and safe in this foreign country. 


\section{Curriculum vitae}

\section{Personal Data}

$\begin{array}{ll}\text { Name: } & \text { Wen Hu } \\ \text { Date of birth: } & 11.04 .1984 \\ \text { Place of birth: } & \text { Anqing, China } \\ \text { Nationality: } & \text { Chinese } \\ \text { Contact information: } & \text { Philipp-Reis 5, 37075 Göttingen, Germany } \\ \text { Mail address: } & \text { huwen.h@gmail.com } \\ \text { Mobile phone No: } & \text { 0176-82087559 }\end{array}$

\section{Education}

10/2007-09/2010 Ph.D in the Neuroscience Program of International Max-Planck Research School, University of Göttingen, Germany, performed at the German Primate Center in the Laboratory of Clinical Neurobiology.

$09 / 2006-09 / 2007$ M.Sc in the Neuroscience Program of International Max-Planck Research School, University of Göttingen, Germany.

09/2002 - 07/2006 B.Sc in Faculty of Life Science, University of Science and Technology of China, P.R.China

\section{$\underline{\text { Awards }}$}

- 2006-2007: Stipend International Max Planck Research School

- 2007-2008: Lichtenberg PhD scholarship awarded by University of Göttingen

- 2008-2010: PhD scholarship awarded by German Primate Center 


\section{Publications}

\section{Papers:}

Hu W, Zhang M, Czéh B, Flügge G, Zhang W (2010).

Stress Impairs GABAergic Network Function in the Hippocampus by Activating Nongenomic Glucocorticoid Receptors and Affecting the Integrity of the Parvalbumin-Expressing Neuronal Network. Neuropsychopharmacology 35: 1693-707.

Hu W, Zhang M, Czéh B, Zhang W, Flügge G (2011) Chronic Restraint Stress Impairs Endocannabinoid Mediated Suppression of GABAergic signaling in the Hippocampus of Adult Male Rats. Brain Research Bulletin, in press.

\section{Abstracts:}

Hu W, Flügge G, Czéh B

Localization of the GABAB (R2) receptor, of plasma membrane GABA transporter type1(GAT1) and vesicular GABA transporter (VGAT) in rat hippocampus by immunocytochemistry.

FENS 2008

Hu W, Zhang M, Czéh B, Zhang W, Flügge G

Chronic restraint stress impairs endocannabinoid mediated suppression of GABA release in the rat hippocampus

Germany Neuroscience Meeting 2009

Hu W, Zhang M, Czéh B, Zhang W, Flügge G

Stress impairs hippocampal GABAergic network function through nongenomic glucocorticoid receptors and affects the integrity of the parvalbumin-expressing neuronal network.

FENS 2010 